\title{
CONTRIBUTION TO THE KNOWLEDGE OF THE LICHEN BIOTA OF BOLIVIA. 5
}

\author{
Adam Flakus, Harrie J. M. Sipman, Kerstin Bach, Pamela Rodriguez \\ Flakus, KerRy KnUdsen, Teuvo Ahti, Ulf Schiefelbein, ZdenĚK Palice, \\ AgnieszKa JabŁońsKa, Magdalena Oset, Rosa I. Meneses Q. \\ \& MARTIN KUKWA ${ }^{1}$
}

\begin{abstract}
This paper presents new records of 180 lichen species from Bolivia; 103 are new national records. Three species, Bryonora curvescens (Mudd) Poelt (also from Ecuador), Lepraria elobata Tønsberg and Pyrenula laetior Müll. Arg., are reported for the first time from the Southern Hemisphere, and five, Bathelium aff. sphaericum (C. W. Dodge) R. C. Harris, Lepraria jackii Tønsberg, Psiloparmelia arhizinosa Hale, Szczawinskia tsugae A. Funk and Trinathotrema lumbricoides (Sipman) Sipman \& Aptroot, are new to South America. To complement the rather poorly recognized distribution of some species, five of the taxa reported here are also new to Ecuador. Notes on distribution and chemistry are provided for most species, and some, especially those belonging to taxonomically critical groups, are discussed in greater detail.
\end{abstract}

Key words: biogeography, Ecuador, lichenized fungi, Neotropics, South America

Adam Flakus, Laboratory of Lichenology, W. Szafer Institute of Botany, Polish Academy of Sciences, Lubicz 46, 31-512 Kraków, Poland

Harrie J. M. Sipman, Botanischer Garten \& Botanisches Museum Berlin Dahlem, Königin-Luise-Strasse 6-8, D-14191 Berlin, Germany

Kerstin Bach, Fachbereich Geographie, Philipps-Universität Marburg, Deutschhausstr. 10, D - 35032 Marburg, Germany

Pamela Rodriguez Flakus, Department of Botany and Molecular Evolution, Senckenberg Forschungsinstitut und Naturmuseum, Senckenberganlage 25, D-60325, Frankfurt am Main, Germany; Herbario Nacional de Bolivia, Instituto de Ecología, Universidad Mayor de San Andrés, Calle 27, Cota Cota, Casilla 10077, La Paz, Bolivia

Kerry Knudsen, The Herbarium, Department of Botany \& Plant Sciences, University of California, 2117 Batchelor Hall, Riverside, California 92521, U.S.A.

Teuvo Ahti, Botanical Museum, Finnish Museum of Natural History, P.O. Box 7, FI-00014 University of Helsinki, Finland Ulf Schiefelbein, Blücherstr. 71, D-18055 Rostock, Germany

Zdeněk Palice, Institute of Botany, Czech Academy of Sciences, CZ-252-43 Prûhonice \& Department of Botany, Faculty of Natural Sciences, Charles University, Benátská 2, CZ-128-01, Praha 2, Czech Republic

Rosa I. Meneses Q., Herbario Nacional de Bolivia, Agreement Instituto de Ecología - Museo Nacional de Historia Natural, Calle 27, Cota Cota, Casilla 10077, La Paz, Bolivia

Agnieszka Jabłońska, Martin Kukwa \& Magdalena Oset, Department of Plant Taxonomy and Nature Conservation, University of Gdańsk, Wita Stwosza 59, 80-308 Gdańsk, Poland; e-mail: dokmak@ug.edu.pl

\section{INTRODUCTION}

Bolivia has the highest ecosystem diversity in South America (Josse et al. 2003). Consequently, we can assume that its lichen biota is very rich, and the number of species should be at least as high as in countries as Brazil, Colombia, Peru or Venezuela (Lücking et al. 2009). Recently,

\footnotetext{
${ }^{1}$ Corresponding author
}

considerable lichenological exploration has been undertaken in the country, mostly in years 2010 2013 (e.g., Krzewicka \& Flakus 2010; Flakus \& Kukwa 2012a, b; Flakus et al. 2011a, 2012a, b; Knudsen et al. 2012; Kukwa et al. 2012, 2013), with a preliminary overview of lichenized and lichenicolous fungi provided by Rodriguez Flakus et al. (2013). However, it appears that our 
understanding of lichen diversity and distribution in Bolivia remains far from complete, and interesting discoveries are continuously being made.

This is the fifth part of our contribution to the understanding of Bolivian lichens based on recent collections by the authors and the continuing revision of herbarium material, mainly from $B$ and LPB. Here, we present new records of many species, including numerous first reports for the country.

\section{MATERIAL AND METHODS}

Specimens are housed at B, BOLV, GOET, KRAM, LPB, PRA, QCA, UGDA, UPS (acronyms after Thiers 2012) and the private herbaria of A. Flakus and U. Schiefelbein. Thin layer chromatography (TLC) was used to identify the secondary lichen metabolites necessary for the recognition of some groups of lichens; methods follow Culberson and Kristinsson (1970) and Orange et al. (2001). The symbol ( \pm ) indicates that a particular substance was absent in some specimens only. In some cases, the fluorescence of the thallus in ultraviolet light (UV) was checked and spot tests were made using $\mathrm{K}(10 \%$ water solution of potassium hydroxide) and $\mathrm{C}$ (commercial bleach).

Notes on general distribution are presented only for those species reported as new to Bolivia. New national records are marked with an asterisk $\left(^{*}\right)$, those new to South America with two $\left(^{* *}\right)$ and those new to the Southern Hemisphere with three $\left({ }^{* * *}\right)$.

The following abbreviations are used: $A F-\mathrm{A}$. Flakus; $J Q-$ J. Quisbert; $K B-\mathrm{K}$. Bach; $M K-\mathrm{M}$. Kukwa; $O P$ - O. Plata; $P R-$ P. Rodriguez Flakus; $R I M$ - R. I. Meneses; US - U. Schiefelbein; $Z P-\mathrm{Z}$. Palice; NP - National Park; AMBORÓ - Parque Nacional y Área Natural de Manejo Integrado Amboró; APOLOBAMBA - Área Natural de Manejo Integrado Nacional Apolobamba; E.B. BENI - Reserva de la Biosfera Estación Biológica del Beni; CARRASCO Parque Nacional Carrasco; COTAPATA - Parque Nacional y Área Natural de Manejo Integrado Cotapata; KAA-IYA- Parque Nacional y Área Natural de Manejo Integrado Kaa-Iya del Gran Chaco; SAJAMA - Parque Nacional Sajama; TARIQUÍA - Reserva Nacional de Flora y Fauna Tariquía; TUNI-CONDORIRI - Parque Nacional Tuni Condoriri (abbreviations of protected areas according SERNAP Bolivia; http://www.sernap. gob.bo).

\section{RESULTS}

New Bolivian records of 180 taxa are presented in this paper; 103 species are new to the country and 5 are new to Ecuador (Bryonora curvescens, Chaenotheca chlorella, C. sphaerocephala, Crocynia gossypina and Trichothelium caudatum). Five species are newly recorded from South America, and three are new to the Southern Hemisphere. Seventeen genera are reported for the first time from Bolivia: Bacidiopsora Kalb, Bryonora Poelt, Chaenotheca (Th. Fr.) Th. Fr., Cryptodiscus Corda, Eschatogonia Trevis., Gyalidea Lettau ex Vězda, Heterocyphelium Vain., Hyperphyscia Müll. Arg., Ingvariella Guderley \& Lumbsch, Letrouitia Hafellner \& Bellem., Megaspora (Clauzade \& Cl. Roux) Hafellner \& V. Wirth, Physcidia Tuck., Polymeridium (Müll. Arg.) R.C. Harris, Pseudopyrenula Müll. Arg., Sipmaniella Kalb, Szczawinskia A. Funk and Tetramelas Norman.

\section{Acarospora boliviana H. Magn.}

This species has been reported in Bolivia by Magnusson (1929) and Weber (1968) and from one locality by Knudsen et al. (2008).

CHEMISTRY. Epanorin and rhizocarpic acid $( \pm)$.

SPECIMENS EXAMINED. BOLIVIA. DEPT. COCHABAMBA. Prov. Quillacollo, area of Incarraya-Sipe Sipe, $17^{\circ} 29^{\prime} 25^{\prime \prime} \mathrm{S}, 66^{\circ} 22^{\prime} 09^{\prime \prime} \mathrm{W}, 3146 \mathrm{~m}$, semi-desert open area, saxicolous, 17 Dec. 2004, AF 4975 (KRAM, LPB); DEPT. LA PAZ. Prov. Murillo, near Cumbre pass, $16^{\circ} 21^{\prime} 59^{\prime \prime} \mathrm{S}, 68^{\circ} 02^{\prime} 37^{\prime \prime} \mathrm{W}, 4604 \mathrm{~m}$, high Andean Puna, saxicolous, 20 Dec. 2009, AF 16307.1 \& PR (LPB); Prov. Omasuyos, Tajani, near Achacachi, $15^{\circ} 36^{\prime} 49^{\prime \prime} \mathrm{S}$, $69^{\circ} 04^{\prime} 02^{\prime \prime} \mathrm{W}, 3869 \mathrm{~m}$, Puna Húmeda, saxicolous, 6 July 2010, AF 17792 \& PR (KRAM, LPB); DEPT. Potosí. Prov. Daniel Campos, Puerto Chuvica, near Salar Uyuni, $20^{\circ} 36^{\prime} 06^{\prime \prime} \mathrm{S}, 67^{\circ} 35^{\prime} 50^{\prime \prime} \mathrm{W}, 3670 \mathrm{~m}$, open semi-desert high Andean area, saxicolous, 4 Dec. 2009, AF 14700 \& PR (KRAM, LPB); Prov. Nor Lípez, Pinturas Rupestres, near Mallku Villamar, $21^{\circ} 46^{\prime} 20^{\prime \prime} \mathrm{S}, 67^{\circ} 29^{\prime} 05^{\prime \prime} \mathrm{W}$, $4038 \mathrm{~m}$, open semi-desert high Andean area, saxicolous, 6 Dec. 2009, $A F 14762 \&$ \&R (KRAM, LPB).

\section{*Acarospora brouardii B. de Lesd.}

This species has been reported in South America from Argentina and Venezuela. It is also known from North America (Knudsen et al. 2008). 
SPECIMENS EXAMINED. BOLIVIA. DEPT. ORURo. Prov. Sajama, SAJAMA, ladera de la quebrada Kohniri, $18^{\circ} 07^{\prime} 07.1^{\prime \prime} \mathrm{S}, 68^{\circ} 56^{\prime} 06.5^{\prime \prime} \mathrm{W}, 4309 \mathrm{~m}$, bosque de Polylepis tarapacana en una ladera con piedras grandes, on rock, 21 Nov. 2007, RIM et al. 4384 (B, LPB).

\section{*Acarospora chrysops (Tuck.) H. Magn.}

In South America this species has been found in Brazil, Columbia and Venezuela; it is also known from the Galapagos Islands, and it extends northward into Central America and the U.S.A. (Knudsen et al. 2008).

Specimens eXamined. BOLIVIA. Dept. La PaZ. Prov. Camacho, al oeste de la ciudad de La Paz cerca de la comunidad de Ulla Ulla, en el cerro Wilamuku, $15^{\circ} 01^{\prime} 36.9^{\prime \prime} \mathrm{S}, 69^{\circ} 11^{\prime} 52.9^{\prime \prime} \mathrm{W}, 4770 \mathrm{~m}$, vegetación Altoandina con rocas, on rock, 4 Dec. 2007, PR 250 (B, LPB).

Acarospora obpallens (Nyl. ex Hasse) Zahlbr.

Previously reported from one locality in Bolivia (Knudsen et al. 2008).

CHEMISTRY. Gyrophoric and lecanoric acids.

SPECIMENS EXAMINED. BOLIVIA. DEPT. Potosí. Prov. Nor Lípez, near volcán Ollagüe, $21^{\circ} 17^{\prime} 13^{\prime \prime} \mathrm{S}$, $68^{\circ} 05^{\prime} 32^{\prime \prime} \mathrm{W}, 4095 \mathrm{~m}$, open semi-desert high Andean area, saxicolous, 5 Dec. 2009, AF $14736.2 \& P R$ (KRAM, LPB); Pinturas Rupestres, near Mallku Villamar, $21^{\circ} 46^{\prime} 20^{\prime \prime} \mathrm{S}, 67^{\circ} 29^{\prime} 05^{\prime \prime} \mathrm{W}, 4038 \mathrm{~m}$, open semidesert high Andean area, saxicolous, 6 Dec. 2009, $A F$ 14764, 14766, 14792, $14794 \&$ \&R (KRAM, LPB).

\section{Acarospora rhabarbarina Hue}

This species has been reported in Bolivia by Magnusson (1929) and Weber (1968) and recently from a few localities by Knudsen et al. (2008).

CHEMISTRY. Rhizocarpic acid and epanorin $( \pm)$.

SPECIMENS EXAmined. BOLIVIA. Dept. La PAZ. Prov. Franz Tamayo, APOLOBAMBA, Socondori Chico near Ulla Ulla, $15^{\circ} 00^{\prime} 38^{\prime \prime} \mathrm{S}, 69^{\circ} 13^{\prime} 48^{\prime \prime} \mathrm{W}, 4479 \mathrm{~m}$, high Andean open vegetation, saxicolous, 4 July 2010, $A F$ 17482 \& PR (KRAM, LPB); Prov. Los Andes, an der Str. La Paz - Tiahuanacu, 3970 m, 14 June 1981, M. Liberman 295 (B); DePT. ORURo. Prov. Sajama, SAJAMA, Aguas Calientes, near Sajama, $18^{\circ} 05^{\prime} 29^{\prime \prime}$, $69^{\circ} 02^{\prime} 28^{\prime \prime} \mathrm{W}, 4448 \mathrm{~m}$, Puna Sureña, shrubland dominated by Baccharis, saxicolous, 18 June 2010, $A F 16523 \& P R$ (KRAM, LPB); Huincurata, near Sajama, $18^{\circ} 07^{\prime} 01^{\prime \prime}$, $68^{\circ} 58^{\prime} 00^{\prime \prime} \mathrm{W}, 4301 \mathrm{~m}$, Puna Sureña, Polylepis forest, saxicolous, 19 June 2010, $A F 16563 \& P R$ (LPB); Jecha K'ala, $18^{\circ} 09^{\prime} 52^{\prime \prime} \mathrm{S}, 68^{\circ} 49^{\prime} 08^{\prime \prime} \mathrm{W}, 4184 \mathrm{~m}$, Puna Sureña, grassland vegetation, saxicolous, 20 June 2010, $A F 16717,16608 \& P R$ (KRAM, LPB, herb. Flakus); DePt. Potosí. Prov. Daniel Campos, Puerto Chuvica, near Salar Uyuni, $20^{\circ} 36^{\prime} 06^{\prime \prime} \mathrm{S}, 67^{\circ} 35^{\prime} 50^{\prime \prime} \mathrm{W}, 3670 \mathrm{~m}$, open semi-desert high Andean area, saxicolous, 4 Dec. 2009, AF 14697, 14718 \& PR (KRAM, LPB); Prov. Nor Lípez, Pinturas Rupestres, near Mallku Villamar, $21^{\circ} 46^{\prime} 20^{\prime \prime} \mathrm{S}, 67^{\circ} 29^{\prime} 05^{\prime \prime} \mathrm{W}, 4038 \mathrm{~m}$, open semi-desert high Andean area, saxicolous, 6 Dec. 2009, AF 14737, $14797 \& P R$ (KRAM, LPB).

\section{Acarospora rouxii K. Knudsen, Elix \& Reeb}

Previously known from one locality in Bolivia (Knudsen 2007; Knudsen et al. 2008).

So far this species has been known to produce norstictic acid in addition to rhizocarpic acid and epanorin (Knudsen 2007; Knudsen et al. 2008). However, one morphologically identical specimen (PR 242) contains stictic acid and related substances, with only a trace of norstictic acid.

CHEMISTRY. Rhizocarpic acid, epanorin, norstictic and connorstictic acid, or rhizocarpic, norstictic (trace), stictic, menegazziaic, cryptostictic and constictic acids.

SPECIMENS EXAMINED. BOLIVIA. DePt. LA PAZ. Prov. Bautista Saavedra, APOLOBAMBA, near Taypi Cañuma, $15^{\circ} 03^{\prime} 20^{\prime \prime} \mathrm{S}, 69^{\circ} 09^{\prime} 07^{\prime \prime} \mathrm{W}, 4506 \mathrm{~m}$, high Andean open vegetation, on sandstone, 5 July 2010, AF 17496 , $17555 \&$ \&R (KRAM, LPB); Prov. Camacho, al oeste de la ciudad de La Paz cerca de la comunidad de Ulla Ulla, en el cerro Wilamuku, $15^{\circ} 01.615^{\prime} \mathrm{S}, 69^{\circ} 11.882^{\prime} \mathrm{W}$, 4770 m, vegetación Altoandina, sobre roca, 4 Dec. 2007, PR 242 (B, LPB); Prov. Franz Tamayo, APOLOBAMBA , near Puyo Puyo, $14^{\circ} 56^{\prime} 55^{\prime \prime} \mathrm{S}, 6^{\circ} 07^{\prime} 58^{\prime \prime} \mathrm{W}, 4888 \mathrm{~m}$, high Andean open vegetation, on schist rock, 5 July 2010, AF 17579, 17609, $17630 \&$ \&R (KRAM, LPB).

\section{*Acarospora strigata (Nyl.) Jatta}

The species was reported in South America from Argentina and Chile, as well as Asia and western North America (Knudsen et al. 2008).

CHEMISTRY. No lichen substances detected by TLC. 
Specimens examined. BOLIVIA. Dept. Potosí. Prov. Daniel Campos, Salar Uyuni, Isla del Incahuasi, $20^{\circ} 14^{\prime} 27^{\prime \prime} \mathrm{S}, 67^{\circ} 37^{\prime} 39^{\prime \prime} \mathrm{W}, 3667 \mathrm{~m}$, open semi-desert high Andean area, on calcareous rock, 3 Dec. 2009, $A F$ $14677 \& P R$ (KRAM, LPB).

\section{Acarospora trachyticola (Müll. Arg.) Hue}

This is the fifth locality of the species in Bolivia (Knudsen et al. 2012).

Chemistry. No lichen substances detected by TLC.

Specimens examined. BOLIVIA. Dept. LA PAZ. Prov. Bautista Saavedra, APOLOBAMBA, near Taypi Cañuma, $15^{\circ} 03^{\prime} 20^{\prime \prime} \mathrm{S}, 69^{\circ} 09^{\prime} 07^{\prime \prime} \mathrm{W}, 4506 \mathrm{~m}$, high Andean open vegetation, saxicolous, 5 July 2010, $A F 17500$ \& PR (KRAM, LPB).

\section{Acarospora xanthophana H. Magn.}

Previously known in Bolivia mostly from old records (Nylander 1861; Magnusson 1929; Weber 1968; Knudsen et al. 2008).

CHEMISTRY. Epanorin $( \pm)$ and rhizocarpic acid $( \pm)$.

SPeCimens eXamined. BOLIVIA. Dept. La PAZ. Prov. Manco Kapac, near Copacabana, Mt. Horca del Inca, $16^{\circ} 10^{\prime} 15^{\prime \prime} \mathrm{S}, 69^{\circ} 05^{\prime} 05^{\prime \prime} \mathrm{W}, 3974 \mathrm{~m}$, high Andean Puna, on volcanic rock, 18 June 2006, $A F$ 8621, 8658 \& 8660 (KRAM, LPB); DePT. ORURO. Prov. Sajama, SAJAMA, Ladera de la Quebrada Kohniri, 4309 m, $18^{\circ} 07^{\prime} 07^{\prime \prime} \mathrm{S}, 68^{\circ} 56^{\prime} 06^{\prime \prime} \mathrm{W}$, bosque de Polylepis tarapacana con piedras grandes, on rock, 21 Nov. 2007, RIM et al. 4371 (B, LPB); DEPT. Potosí. Prov. Daniel Campos, Puerto Chuvica, near Salar Uyuni, $20^{\circ} 36^{\prime} 06^{\prime \prime}$, $67^{\circ} 35^{\prime} 50^{\prime \prime} \mathrm{W}, 3670 \mathrm{~m}$, open semi-desert high Andean area, on volcanic rock, 4 Dec. 2009, AF 14687, 14711, $14720 \& P R$ (KRAM, LPB); Prov. Daniel Campos, Salar Uyuni, isla Incahuasi, 20 $14^{\prime} 27^{\prime \prime} \mathrm{S}, 67^{\circ} 37^{\prime} 39^{\prime \prime} \mathrm{W}, 3667 \mathrm{~m}$, open semi-desert high Andean area, on soil or volcanic rocks, 3 Dec. 2009, AF 14671, 14679, $14684 \& P R$ (KRAM, LPB); Prov. Nor Lípez, near volcán Ollagüe, $21^{\circ} 17^{\prime} 13^{\prime \prime} \mathrm{S}, 68^{\circ} 05^{\prime} 32^{\prime \prime} \mathrm{W}, 4095 \mathrm{~m}$, open semi-desert high Andean area, on volcanic rock, 5 Dec. 2009, $A F$ $14727,14728,14731 \& P R$ (KRAM, LPB).

\section{Anzia parasitica (Fée) Zahlbr.}

Previously known from only one locality in Bolivia (Flakus et al. 2011a).

\section{CHEMISTRY. Divaricatic acid.}

Specimens examined. BOliVia. Dept. Santa CruZ. Prov. Caballero, near Siberia, $17^{\circ} 49^{\prime} 38^{\prime \prime} \mathrm{S}$, $64^{\circ} 44^{\prime} 45^{\prime \prime} \mathrm{W}, 3950 \mathrm{~m}$, open Yungas cloud forest, epiphytic, 16 Aug. 2012, MK 11450 (LPB, UGDA).

\section{*Arthrorhaphis alpina (Schaer.) R. Sant.}

This species is widely distributed and occurs in all continents except Africa (Feuerer 2013). In South America it is known from Chile, Colombia, Ecuador and Venezuela (Arvidsson 1991; Marcano et al. 1996; Galloway \& Quilhot 1998; Sipman et al. 2008).

Specimens eXamined. BOLIVIA. Dept. Cochabamba. Prov. Carrasco, CARRASCO, Koricaza, $17^{\circ} 33^{\prime} 21^{\prime \prime} \mathrm{S}, 65^{\circ} 16^{\prime} 29^{\prime \prime} \mathrm{W}, 2950 \mathrm{~m}$, Páramo Yungueño, terricolous, 18 Aug. 2012, AF 24619 (KRAM, LPB); La Cumbre, El Camino de las Nubes, $17^{\circ} 17^{\prime} 46^{\prime \prime} \mathrm{S}$, $65^{\circ} 43^{\prime} 56^{\prime \prime} \mathrm{W}, 4100 \mathrm{~m}$, Páramo Yungueño, terricolous, 19 Aug. 2012, AF 24689 (LPB, herb. Flakus). ECUADOR. Prov. Cotopaxi, NP Cotopaxi, NNE slope of volcano Cotopaxi, $00^{\circ} 36^{\prime} 50^{\prime \prime} \mathrm{S}, 78^{\circ} 24^{\prime} 15^{\prime \prime} \mathrm{W}, 3800 \mathrm{~m}$, eroding slope above a brook, 22 June 1999, ZP 2565, 2889 (PRA, QCA); ibidem, above the valley of Quebrada Tañiloma, $00^{\circ} 39^{\prime} \mathrm{S}, 78^{\circ} 25^{\prime} \mathrm{W}, 4300-4350 \mathrm{~m}$, terricolous, 28 June 1999, ZP 2870 (PRA).

*Astrothelium cinnamomeum (Eschw.) Müll. Arg.

This pantropical species was reported in South America from Brazil, Colombia and the Guyanas (Hekking \& Sipman 1988; Cáceres 2007; Sipman et al. 2008).

CHEMISTRY. Thallus UV -; pseudostroma with a superficial, orange, UV+ orange pigment.

Specimens EXAmined. BOLIVIA. Dept. Beni. Prov. Cercado, Casa del Tigre, near Chuchini, 1443'17"S, $64^{\circ} 56^{\prime} 53^{\prime \prime} \mathrm{W}, 160 \mathrm{~m}$, lowland Amazon forest, on bark, 31 Aug. 2012, MK 11985 (LPB, UGDA); DEPT. LA PAZ. Prov. Iturralde, forest above Tumupasa, $14^{\circ} 08^{\prime} 51^{\prime \prime} \mathrm{S}$, $67^{\circ} 53^{\prime} 34^{\prime \prime} \mathrm{W}, 350 \mathrm{~m}$, Subandean Amazon forest, on bark of tree, 31 Aug. 2008, MK 7093 (LPB, UGDA).

\section{Aspidothelium cinerascens Vain.}

This species has been reported in Bolivia by Flakus and Wilk (2006). 
Specimen eXamined. BOLIVIA. DePt. LA PaZ. Prov. Nor Yungas, Coroico, Sol y Luna hotel, $1870 \mathrm{~m}$, $16^{\circ} 11^{\prime} 56^{\prime \prime} \mathrm{S}, 67^{\circ} 43^{\prime} 26^{\prime \prime} \mathrm{W}$, gardens with trees, on bark, 13 Dec. 2011, MK 10800 (LPB).

Aspidothelium fugiens (Müll. Arg.) R. Sant.

So far one locality was known from Bolivia (Flakus et al. 2012a).

Specimens eXamined. BOLIVIA. DePt. LA PaZ. Prov. Nor Yungas, Coroico, Sol y Luna hotel, $1870 \mathrm{~m}$, $16^{\circ} 11^{\prime} 56^{\prime \prime} \mathrm{S}, 67^{\circ} 43^{\prime} 26^{\prime \prime} \mathrm{W}$, gardens with trees, on bark, 13 Dec. 2011, $M K 10801$ (LPB, UGDA).

\section{${ }^{*}$ Bacidiopsora microphyllina Kalb}

This recently described species was known from Costa Rica, Brazil, Panama, Venezuela, as well as Cameroon and Réunion (Kalb 2004; Cáceres 2007).

CHEMISTRY. Homosekikaic and sekikaic acids.

SPECIMENS EXAMINED. BOLIVIA. DePT. COCHABAMBA. Prov. Carrasco, CARRASCO, Ch'iqta rumi, $17^{\circ} 28^{\prime} 44^{\prime \prime} \mathrm{S}, 65^{\circ} 17^{\prime} 06^{\prime \prime} \mathrm{W}, 2120 \mathrm{~m}$, Yungas cloud forest, corticolous, 21 July 2008, $A F$ 10257, MK \& PR (KRAM, LPB); near Sehuencas, $17^{\circ} 30^{\prime} 12^{\prime \prime} \mathrm{S}, 65^{\circ} 16^{\prime} 30^{\prime \prime} \mathrm{W}$, $2220 \mathrm{~m}$, Yungas cloud forest, corticolous, 21 July 2008, $A F$ 10519, $M K \&$ \&R (KRAM, LPB); DEPT. LA PAZ. Prov. Nor Yungas, COTAPATA, near Urpuma colony, $16^{\circ} 13^{\prime} 20^{\prime \prime} \mathrm{S}, 67^{\circ} 52^{\prime} 34^{\prime \prime} \mathrm{W}, 1989 \mathrm{~m}$, Yungas montane forest, corticolous, 30 June 2010, $A F$ 17188.2 \& PR (KRAM, LPB).

\section{*Bacidiopsora orizabana (Vain.) Kalb}

This rare lichen has been reported from Brazil and Mexico in the Neotropics (Kalb 2004; Cáceres 2007), and also from tropical Asia (Thailand and Taiwan) (Aptroot et al. 2007).

Specimens examined. BOliVia. Dept. Santa Cruz. Prov. Cordillera, near Peto Blanco, park guard's station, $18^{\circ} 48^{\prime} 25^{\prime \prime} \mathrm{S}, 60^{\circ} 14^{\prime} 54^{\prime \prime} \mathrm{W}, 330 \mathrm{~m}$, transition Chiquitano-Chaqueño forest, corticolous, 6 Dec. 2011, AF 23768 (KRAM, LPB); KAA-IYA, Bañados del Izozog, near río Parapetí, $18^{\circ} 28^{\prime} 40^{\prime \prime} \mathrm{S}, 62^{\circ} 05^{\prime} 03^{\prime \prime} \mathrm{W}$, 320 m, Ribereño forest, corticolous, 2 Dec. 2010, $A F$ $18881 \& J Q$ (KRAM, LPB).
Baeomyces rufus (Huds.) Rebent.

This is the second record from Bolivia (Flakus et al. 2012a).

SPECIMEN EXAMINED. BOLIVIA. DEPT. COCHABAMBA. Prov. Carrasco, CARRASCO, Koricaza, $17^{\circ} 33^{\prime} 21^{\prime \prime} \mathrm{S}, 65^{\circ} 16^{\prime} 29^{\prime \prime} \mathrm{W}, 2950$ m, Páramo Yungueño, terricolous, 18 Aug. 2012, AF 24620 (LPB).

*Bathelium madreporiforme (Eschw.) Trevis.

According to Aptroot et al. (2008) B. madreporiforme is a Neotropical lichen, but it was also reported from Australia and Thailand (McCarthy 1993; Wolseley et al. 2002; Aptroot 2009). In the Neotropics it is known from Brazil, Colombia, Costa Rica, French Guiana, Paraguay and the U.S.A. (Florida) (Malme 1924; Hekking \& Sipman 1988; Harris 1995; Cáceres 2007; Aptroot et al. 2008; Sipman et al. 2008).

Two of the samples cited here differ from the description provided in Aptroot et al. (2008) by having a yellow, UV+ orange thallus. However, according to Harris (1995; notes under Trypethelium variolosum Ach.) almost every lichexanthonecontaining taxon has some lichexanthone-deficient specimens. Further study is required to determine whether these character states merit taxonomic recognition.

ChEMISTRY. Thallus UV+ orange yellow or negative; pseudostroma containing orange, $\mathrm{K}+$ red pigment.

SPECIMENS WITH UV- THALLUS EXAMINED. BOLIVIA. DEPT. SANTA CRUZ. Prov. Cordillera, SSW of Roboré, $18^{\circ} 36^{\prime} 11^{\prime \prime} \mathrm{S}, 59^{\circ} 53^{\prime} 06^{\prime \prime} \mathrm{W}, 320 \mathrm{~m}$, transition Chiquitano-Chaqueño forest, on tree bark, 4 Dec. 2011, MK 10601 (LPB, UGDA).

SPECIMENS WITH UV+ ORANGE THALLUS EXAMined. BOLIVIA. DePt. Beni. Prov. General José Ballivián Segurola, north of San Borja, $14^{\circ} 46^{\prime} 07^{\prime \prime}$, $66^{\circ} 46^{\prime} 51^{\prime \prime} \mathrm{W}, 190 \mathrm{~m}$, lowland Amazon forest surrounded by los Llanos de Moxos savanna, on bark, 3 Sept. 2012, MK 12052 (LPB, UGDA); DEPT. SANTA CRUZ. Prov. Cordillera, near Peto Blanco, $18^{\circ} 48^{\prime} 25^{\prime \prime} \mathrm{S}, 60^{\circ} 14^{\prime} 54^{\prime \prime} \mathrm{W}$, $330 \mathrm{~m}$, transition Chiquitano-Chaqueño forest, on bark, 6 Dec. 2011, MK 10679 (LPB, UGDA). 


\section{*Bathelium mastoideum Afzel. ex Ach.}

This species is known from tropical America and Africa (Harris 1995; Aptroot et al. 2008). In the Neotropics it has been reported from Brazil and Costa Rica (Cáceres 2007; Aptroot et al. 2008).

Chemistry. Thallus UV+ orange-yellow; pseudostroma containing an orange pigment.

Specimens examined. BOliVia. Dept. Santa CRUZ. Prov. Cordillera, $350 \mathrm{~km}$ on the road from Tucavaca to Roboré, $18^{\circ} 37^{\prime} 56^{\prime \prime} \mathrm{S}, 59^{\circ} 36^{\prime} 50^{\prime \prime} \mathrm{W}, 370 \mathrm{~m}$, transition Chiquitano-Chaqueño forest, on twigs, 4 Dec. 2011, MK 10531a (LPB).

\section{${ }^{* *}$ Bathelium aff. sphaericum (C. W. Dodge)}

\section{R. C. Harris}

This specimen is most similar to the African species $B$. sphaericum. However, it differs in having 4-spored asci and pseudostroma with sparse pigment crystals (in $B$. sphaericum the asci are 8 -spored and the pigment crystals are abundant) (Dodge 1953; Letrouit-Galinou 1957; Harris 1995).

CHEMISTRY. Thallus UV-; pseudostroma with sparse crystals of orange-red pigment.

SPeCimens eXamined. BOLIVIA. DePt. La PAZ. Prov. Franz Tamayo, between Apolo and Mapiri, $15^{\circ} 01^{\prime} 50^{\prime \prime} \mathrm{S}, 68^{\circ} 18^{\prime} 33^{\prime \prime} \mathrm{W}, 1120 \mathrm{~m}$, Preandean Amazon forest, on bark, 23 Nov. 2011, MK 10302 (LPB, UGDA).

Brigantiaea leucoxantha (Spreng.) R. Sant. \& Hafellner

Previously recorded from Bolivia by Feuerer (2010).

SPECIMENS EXAMINED. BOLIVIA. DEPT. COCHABAMBA. Prov. Ayopaya, $20 \mathrm{~km}$ de Cocapata hacia Cotacajes, $16^{\circ} 46^{\prime} \mathrm{S}, 66^{\circ} 44^{\prime} \mathrm{W}, 2100 \mathrm{~m}$, bosque semideciduo intervenido, epiphytic, 17 May 1997, KB et al. 119 (LPB); DePT. LA PAZ. Prov. Nor Yungas, Cotapata, hacia Bajo Hornuni, 16¹ $11^{\prime} 06^{\prime \prime} \mathrm{S}, 67^{\circ} 49^{\prime} 30^{\prime \prime} \mathrm{W}, 1520 \mathrm{~m}$, bosque muy húmedo, epiphytic, 13 Oct. 2006, PR et al. 158 (B, LPB); DePt. SANTA CruZ. Prov. Florida, AMBORÓ, above la Yunga, senda Los Helechos, $18^{\circ} 03^{\prime} 30^{\prime \prime} \mathrm{S}, 63^{\circ} 54^{\prime} 36^{\prime \prime} \mathrm{W}, 2330 \mathrm{~m}$, Yungas cloud forest, on bark, 7 June 2011, MK 9780 (LPB, UGDA).

\section{*** Bryonora curvescens (Mudd) Poelt}

This species was known previously from the Northern Hemisphere: Asia (Nepal), Europe (e.g., Austria, Fennoscandia), North America and South America (Venezuela) (Poelt \& Obermayer 1991; Hafellner \& Türk 2001; Santesson et al. 2004; Esslinger 2012).

CHEMISTRY. Not tested by TLC, but red needlelike crystals of norstictic acid formed in $\mathrm{K}$ from apothecial sections.

Specimens examined. BOLIVIA. Dept. La PaZ. Prov. Murillo, near Cumbre pass, $16^{\circ} 21^{\prime} 59^{\prime \prime} \mathrm{S}, 68^{\circ} 02^{\prime} 37^{\prime \prime} \mathrm{W}$, $4604 \mathrm{~m}$, high Andean Puna, on terricolous bryophytes, 20 Dec. 2009, $A F 16315 \&$ PR (LPB). ECUADOR. Prov. Azuay, Área Nacional de Recreación Cajas, laguna Torreadora, $02^{\circ} 46^{\prime} 50^{\prime \prime} \mathrm{S}, 79^{\circ} 13^{\prime} 40^{\prime \prime} \mathrm{W}, 4100 \mathrm{~m}$, over saxicolous bryophytes, 2 Aug. 1999, ZP 3314 (PRA).

\section{Buellia mexicana J. Steiner}

This is the second record of the species from Bolivia (Feuerer \& Sipman 2005).

Specimens examined. BOLIVIA. Dept. La Paz. Prov. Camacho, al oeste de la ciudad de La Paz cerca de la comunidad de Ulla Ulla, en el cerro Wilamuku, $15^{\circ} 02^{\prime} 4.1^{\prime \prime} \mathrm{S}, 69^{\circ} 11^{\prime} 52.7^{\prime \prime} \mathrm{W}, 4739 \mathrm{~m}$, vegetación Altoandina con rocas, on rock, 4 Dec. 2007, PR 228 (B, LPB).

\section{*Bulbothrix suffixa (Stirt.) Hale}

This species occurs in tropical America and South Africa (Hale 1976a). In South America it was found in Brazil, Ecuador, Guyana and Venezuela (Hale 1976a; Fleig \& Grüninger 2000; Nöske \& Sipman 2004; Feuerer 2013).

CHEMISTRY. Not tested by TLC; medulla $\mathrm{C}+$ red; cortex $\mathrm{K}+$ yellow.

Specimens eXamined. BOLIVIA. Dept. La PaZ. Prov. Franz Tamayo, between Apolo and Mapiri, $14^{\circ} 41^{\prime} 50^{\prime \prime} \mathrm{S}, 68^{\circ} 25^{\prime} 07^{\prime \prime} \mathrm{W}, 1510 \mathrm{~m}$, savannah with scattered trees, on bark, 22 Nov. 2011, MK $10216 a$ (LPB, UGDA).

\section{*Bunodophoron macrocarpum (Ohlsson) Wedin}

Previously known from Chile, New Zealand and Tasmania (Wedin 1995), this lichen is 
reported here from its northernmost locality in South America.

Although one of the specimens (MK 11602) was sterile, it was very similar in overall morphology to the fertile collection.

\section{CHEMISTRY. Sphaerophorin.}

Specimens examined. BOLIVIA. Dept. CoCHABAMBA. Prov. Carrasco, CARRASCO, Koricaza, $17^{\circ} 33^{\prime} 21^{\prime \prime} \mathrm{S}, 65^{\circ} 16^{\prime} 29^{\prime \prime} \mathrm{W}, 2950 \mathrm{~m}$, Páramo Yungueño, on ground, 18 Aug. 2012, MK 11712 (LPB, UGDA); DePt. SANTA CRuz. Prov. Caballero, El Camino de Orquídeas, $17^{\circ} 49^{\prime} 50^{\prime \prime} \mathrm{S}, 64^{\circ} 42^{\prime} 11^{\prime \prime} \mathrm{W}, 2415 \mathrm{~m}$, Yungas cloud forest, on tree, 17 Aug. 2012, MK 11602 (LPB, UGDA; sterile specimen).

\section{Byssoloma chlorinum (Vain.) Zahlbr.}

This lichen is already known from several localities in Bolivia (Ferraro 2002; Flakus \& Lücking 2008; Lücking 2008).

Specimens eXAmined. BOLIVIA. DePt. COCHABAMBA. Prov. Carrasco, CARRASCO, near Sehuencas, $17^{\circ} 29^{\prime} 48^{\prime \prime} \mathrm{S}, 65^{\circ} 16^{\prime} 22^{\prime \prime} \mathrm{W}, 2250 \mathrm{~m}$, Yungas forest along the river, on bark, 18 Aug. 2012, MK 11758 a (LPB, UGDA); DePt. Cochabamba. Prov. Chapare, near Incachaca, $17^{\circ} 12^{\prime} 54^{\prime \prime} \mathrm{S}, 65^{\circ} 49^{\prime} 30^{\prime \prime} \mathrm{W}, 2028 \mathrm{~m}$, Yungas cloud forest near road, corticolous, 7 July 2009, $A F$ 13000 (KRAM, LPB).

\section{${ }^{*}$ Calicium hyperelloides Nyl.}

This widely distributed species is known from Africa, southern and eastern Asia, Australia, South America, Oceania, and the southernmost parts of North America and Europe (Tibell 1996). In South America it has been reported from Argentina, Brazil, Colombia, Ecuador, French Guiana, Paraguay, Peru, Uruguay and Venezuela (Tibell 1996; Aptroot 2002; Sipman et al. 2008).

Chemistry. Not tested by TLC; thallus C+ orange.

SPECIMENS EXAMINED. BOLIVIA. DEPT. COCHABAMBA. Prov. Chapare, CARRASCO, near Incachaca, $17^{\circ} 14^{\prime} 13^{\prime \prime} \mathrm{S}, 65^{\circ} 49^{\prime} 02^{\prime \prime} \mathrm{W}, 2294 \mathrm{~m}$, Yungas cloud forest, on wood, 10 June 2006, AF 8259 (KRAM, LPB, UGDA).

\section{${ }^{*}$ Calicium leucochlorum Tuck.}

This Neotropical lichen is already known from the Bahamas, Brazil, Cuba, the Dominican Republic, Paraguay, Peru and the U.S.A. (Florida) (Tibell 1991, 1996; Harris 1995).

CHEMISTRY. Thiophanic acid and an unidentified xanthone (similar in Rf classes to arthothelin); thallus $\mathrm{C}+$ orange.

SPECIMENS EXAMINED. BOLIVIA. DePt. BENI. Prov. Marbán, near San Lorenzo, $15^{\circ} 12^{\prime} 59^{\prime \prime} \mathrm{S}, 64^{\circ} 47^{\prime} 08^{\prime \prime} \mathrm{W}$, $160 \mathrm{~m}$, lowland Amazon forest surrounded by los Llanos de Moxos savanna, on palm, 28 Aug. 2012, MK 11896 (LPB, UGDA).

\section{Calicium salicinum Pers.}

Already known from a single locality in Bolivia (Flakus 2008).

SPECIMENS EXAMINED. BOLIVIA. DEPT. COCHABAMBA. Prov. Chapare, CARRASCO, near Incachaca, $17^{\circ} 14^{\prime} 13^{\prime \prime} \mathrm{S}, 65^{\circ} 49^{\prime} 02^{\prime \prime} \mathrm{W}, 2294 \mathrm{~m}$, Yungas cloud forest, on wood, 10 June 2006, $A F 8259.1$ (LPB); DEPT. LA PAZ. Prov. Iturralde, near Cosmos colony, by the way between Ixiamas and Tumupasa, $14^{\circ} 01^{\prime} 35^{\prime \prime} \mathrm{S}, 67^{\circ} 57^{\prime} 55^{\prime \prime} \mathrm{W}$, $300 \mathrm{~m}$, open area, on wood, 27 July 2008, MK 6756 (LPB, UGDA); Tahua, 13 ${ }^{\circ} 51^{\prime} 47^{\prime \prime} \mathrm{S}, 67^{\circ} 54^{\prime} 32^{\prime \prime} \mathrm{W}$, $230 \mathrm{~m}$, garden, on wood, 29 July 2008, MK 6919 (LPB, UGDA).

\section{Candelaria concolor (Dicks.) Arnold}

Reported from Bolivia by Feuerer et al. (1998) and Canseco et al. (2006), some or all of the previous records may belong to the recently distinguished C. pacifica M. Westb. \& Arup, a widespread taxon known in South America from Chile (Westberg \& Arup 2011).

Specimens eXamined. BOLIVIA. DePt. SAnta Cruz. Prov. Florida, AMBORÓ, below la Yunga, $18^{\circ} 05^{\prime} 59^{\prime \prime} \mathrm{S}, 63^{\circ} 56^{\prime} 55^{\prime \prime} \mathrm{W}, 1650 \mathrm{~m}$, dry forest on the slope, on rock, 8 June 2011, MK $9957 a$ (LPB, UGDA).

\section{Canoparmelia amazonica (Nyl.) Elix \& Hale}

This species has been reported in Bolivia by Hale (1976b) and Flakus et al. (2011a).

CHEMISTRY. Atranorin and protocetraric acid. 
In one specimen, additional unidentified pigments (traces) were detected by TLC.

Specimens examined. BOLIVIA. Dept. La PaZ. Prov. Iturralde, forest above Tumupasa, $14^{\circ} 08^{\prime} 51^{\prime \prime} \mathrm{S}$, $67^{\circ} 53^{\prime} 34^{\prime \prime} \mathrm{W}, 350 \mathrm{~m}$, Subandean Amazon forest, on bark, 31 Aug. 2008, $M K 7002 \& 7090$ (LPB, UGDA).

\section{Canoparmelia caroliniana (Nyl.) Elix \& Hale}

This is the third record from Bolivia (Flakus et al. 2012a).

\section{CHEMISTRY. Perlatolic acid.}

SPECIMENS EXAMINED. BOLIVIA. DEPT. TARIJA. Prov. Aniceto Arce, Filo de Sidras, $22^{\circ} 14^{\prime} 50^{\prime \prime} \mathrm{S}$,

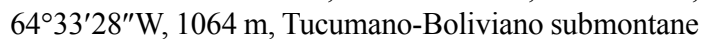
forest, on bark, 22 Nov. 2010, $A F 18675$ (KRAM, LPB, UGDA).

\section{${ }^{*}$ Catapyrenium daedaleum (Kremp.) Stein}

This rather widely distributed species (Prieto et al. 2010) has been recorded in South America from Argentina, Chile and Peru (Breuss 1993; Galloway \& Quilhot 1998).

Specimens eXamined. BOLIVIA. DePt. LA PAZ. Prov. Camacho, al oeste de la ciudad de La Paz cerca de la comunidad de Ulla Ulla, en el cerro Wilamuku, $15^{\circ} 02^{\prime} 04.1^{\prime \prime} \mathrm{S}, 69^{\circ} 11^{\prime} 52.7^{\prime \prime} \mathrm{W}, 4739 \mathrm{~m}$, vegetación Altoandina con rocas, terricolous, 4 Dec. 2007, PR 274 (B, LPB).

\section{${ }^{*}$ Chaenotheca chlorella (Ach.) Müll. Arg.}

This is a widely distributed lichen, especially in temperate latitudes of the Northern and Southern Hemispheres. In Central and South America it is known from Brazil, Chile, Costa Rica and Colombia (Tibell 1996, 1998; Aptroot 2002; Sipman et al. 2008). It is reported here for the first time from Bolivia and Ecuador.

Specimens examined. BOLIVIA. Dept. Santa CruZ. Prov. Caballero, El Rincón, $17^{\circ} 49^{\prime} 33^{\prime \prime} \mathrm{S}$, $64^{\circ} 38^{\prime} 50^{\prime \prime} \mathrm{W}, 2670 \mathrm{~m}$, Yungas cloud forest, on wood, 17 Aug. 2012, MK 11553 (LPB, UGDA). ECUADOR. Prov. Chimborazo, NP Sangay, El Altar, fragment of Gynoxys forest on the crest descending E of Laguna de Collantes ("Yellow Lagoon"), $01^{\circ} 40^{\prime} 10^{\prime \prime} \mathrm{S} 78^{\circ} 25^{\prime} 45^{\prime \prime} \mathrm{W}$,
4150 m, on bark of Gynoxys, 26 Aug. 1999, ZP 3947 (PRA, UPS).

\section{* Chaenotheca sphaerocephala Nádv.}

In South America this species has been reported from Argentina and Chile, but it is probably more common because several previous records of C. brunneola (Ach.) Müll. Arg. are thought to belong to this species (Tibell 1998). A new record for Ecuador.

CHEMISTRY. Barbatic acid (Bolivian material tested). Tibell (1998) also reported obtusatic acid in small concentrations, but this substance was not detected in Bolivian material as only small fragments were taken for TLC.

SPeCimens eXAmined. BOLIVIA. DePt. La PAZ. Prov. Iturralde, forest above Tumupasa, $14^{\circ} 08^{\prime} 51^{\prime \prime} \mathrm{S}$, $67^{\circ} 53^{\prime} 34^{\prime \prime} \mathrm{W}, 350 \mathrm{~m}$, Subandean Amazon forest, on wood, 31 Aug. 2008, MK 7085 (LPB, UGDA); Prov. Larecaja, near Pajonal vilaque, $15^{\circ} 28^{\prime} 02^{\prime \prime} \mathrm{S}, 68^{\circ} 02^{\prime} 12^{\prime \prime} \mathrm{W}, 890 \mathrm{~m}$, Preandean Amazon forest, on bark of tree, 24 Nov. 2011, MK 10396 (LPB, UGDA). ECUADOR. Prov. Loja, NP Podocarpus, Cajanuma ranger station, mountain foggy forest, $04^{\circ} 07^{\prime} \mathrm{S}, 79^{\circ} 10^{\prime} \mathrm{W}, 2800-2850 \mathrm{~m}$, on tree bark, 4 Aug. 1999, ZP 2982 (PRA, UPS); Prov. Carchi, volcán Chíles, along Tulcán-Maldonado road, $c a 0.5 \mathrm{~km}$ ESE from laguna Verde, $00^{\circ} 47.96^{\prime} \mathrm{N}, 77^{\circ} 54.89^{\prime} \mathrm{W}, 4040 \mathrm{~m}$, on roots, 12 July 1999, ZP 4599 (PRA, UPS).

\section{${ }^{*}$ Cladonia andesita Vain.}

This Neotropical species is known from Brazil, Colombia, Ecuador, Peru and Venezuela (Ahti 2000).

Specimens eXAmined. BOLIVIA. DePt. CoCHABAmBA. Prov. Ayopaya, $10 \mathrm{~km}$ de Cocapata hacia Cotacajes, 2800-2850 m, 16 $6^{\circ} 38^{\prime} \mathrm{S}, 66^{\circ} 41^{\prime} \mathrm{W}$, bosque húmedo, 10 May 1997, KB et al. 4 (B, LPB); $20 \mathrm{~km} \mathrm{de}$ Cocapata hacia Cotacajes, $16^{\circ} 46^{\prime} \mathrm{S}, 66^{\circ} 44^{\prime} \mathrm{W}, 2000 \mathrm{~m}$, bosque semideciduo intervenido, saxicolous, 15 May 1997, KB et al. 103 (B, LPB); comunidad de Saila Pata, $3500 \mathrm{~m}, 16^{\circ} 55^{\prime} \mathrm{S}, 66^{\circ} 55^{\prime} \mathrm{W}$, sendero con rocas en bosque siempreverde, saxicolous, 15 Nov. 1997, KB 909 \& I. Jiménez (B, LPB); DEPT. LA PAZ. Prov. Bautista Saavedra, cerro Asunta Pata, $1500 \mathrm{~m}, 15^{\circ} 05^{\prime} \mathrm{S}, 68^{\circ} 29^{\prime} \mathrm{W}$, bosque siempreverde, on decaying wood, 21 June 1997, KB et al. 186 (LPB); $15 \mathrm{~km}$ de Charazani hacia Apolo, $2400 \mathrm{~m}, 15^{\circ} 11^{\prime} \mathrm{S}, 68^{\circ} 52^{\prime} \mathrm{W}$, bosque húmedo secundario 
con rocas, terricolous, 2 July 1997, KB et al. 291 (LPB); ibidem, $3500 \mathrm{~m}, 15^{\circ} 11^{\prime} \mathrm{S}, 68^{\circ} 52^{\prime} \mathrm{W}$, bosque húmedo, terricolous, 4 July 1997, KB et al. 352 (LPB); Prov. Nor Yungas, Coscapa, $c a 4 \mathrm{~km}$ de Cotapata hacia La Paz, $3450-3500 \mathrm{~m}, 16^{\circ} 23^{\prime} \mathrm{S}, 67^{\circ} 53^{\prime} \mathrm{W}$, pastizal con rocas, on wood, saxicolous and terricolous, 9-10 Sept. 1997, $K B$ et al. 463, $464 \& 483$ (B, LPB); San Vincente, $c a$ $1 \mathrm{~km}$ al este de Chuspipata, $3000 \mathrm{~m}, 16^{\circ} 21^{\prime} \mathrm{S}, 67^{\circ} 49^{\prime} \mathrm{W}$, on saxicolous bryophytes, 21 Sept. 1997, KB et al. 581 (LPB); DEPT. SANTA CRUZ. Prov. Florida, Refugio Los Volcanes, $1000 \mathrm{~m}, 18^{\circ} 06^{\prime} \mathrm{S}, 63^{\circ} 36^{\prime} \mathrm{W}$, ladera arenosa de una pared rocosa, saxicolous, 4 Oct. 1997, KB et al. 643 (LPB).

\section{${ }^{*}$ Cladonia corymbites Nyl.}

In South America this species is known from Colombia, Ecuador, Peru and Venezuela; it is also known from Mexico, Costa Rica, Panama and the Caribbean, where it is apparently more common (Ahti 2000).

SPECIMENS EXAMINED. BOLIVIA. DEPT. COCHABAMBA. Prov. Chapare, CARRASCO, $130 \mathrm{~km}$ del camino antiguo de Cochabamba a Villa Tunari, $2200 \mathrm{~m}$, $17^{\circ} 07^{\prime} \mathrm{S}, 65^{\circ} 36^{\prime} \mathrm{W}$, bosque húmedo con paredes rocosas, terricolous, 10 Oct. 1997, KB et al. 702 (LPB); ibidem, $132 \mathrm{~km}$ del camino antiguo de Cochabamba a Villa Tunari, bosque siempreverde, epiphytic, 11 Oct. 1997, KB et al. 845 (LPB); DEPT. SANTA CRUZ. Prov. Florida, Refugio Los Volcanes, $1000 \mathrm{~m}, 18^{\circ} 06^{\prime} \mathrm{S}, 63^{\circ} 36^{\prime} \mathrm{W}$, bosque semi-deciduo, on decaying wood, 3 Oct. 1997, $K B$ et al. 626 (B, LPB, with a specimen of Cladonia subradiata).

\section{Cladonia crispata (Ach.) Flot.}

This is the second collection of $C$. crispata from Bolivia (Flakus et al. 2012a).

Specimen eXamined. BOLIVIA. Dept. La PaZ. Prov. Bautista Saavedra, $15 \mathrm{~km}$ de Charazani hacia Apolo, $2400 \mathrm{~m}, 15^{\circ} 11^{\prime} \mathrm{S}, 68^{\circ} 52^{\prime} \mathrm{W}$, pastos con arbustos al lado del río Camata, terricolous, 1 July 1997, KB et al. 280 (LPB).

\section{Cladonia farinophylla Ahti}

Only recently reported from two localities in Bolivia (Flakus et al. 2012a).

Chemistry. Fumarprotocetraric acid and related substances.
Specimens eXamined. BOLIVIA. Dept. LA PAZ. Prov. Nor Yungas, COTAPATA, Santa Catalina, $16^{\circ} 11^{\prime} 12^{\prime \prime} \mathrm{S}, 67^{\circ} 52^{\prime} 07^{\prime \prime} \mathrm{W}$, Yungas montane forest, on soil, 22 May 2011, US (LPB, herb. Schiefelbein 2945).

\section{Cladonia furcata (Huds.) Schrad.}

This is the third Bolivian record of this species (Ahti 2000; Flakus et al. 2012a).

SPeCimens eXamined. BOlivia. Dept. CoChABAmBa. Prov. Ayopaya, comunidad de Saila Pata, $3700 \mathrm{~m}, 16^{\circ} 55^{\prime} \mathrm{S}, 66^{\circ} 55^{\prime} \mathrm{W}$, quebrada en Puna con bosque siempreverde de Polylepis, terricolous, 15 Nov. 1997, KB 917 \& I. Jiménez (B, LPB).

\section{Cladonia macilenta Hoffm.}

Previously reported from Boliva by Flakus et al. (2012a).

CHEMISTRY. Barbatic acid (one specimen examined by TLC).

Specimens eXAmined. BOLIVIA. DePt. CoCHABAMBA. Prov. Ayopaya, $10 \mathrm{~km}$ de Cocapata hacia Cotacajes, $2800-2850 \mathrm{~m}, 16^{\circ} 38^{\prime} \mathrm{S}, 66^{\circ} 41^{\prime} \mathrm{W}$, bosque húmedo, epiphytic, 10 May 1997, KB et al. $11 \mathrm{c} \& 18 \mathrm{c}$ (B, LPB); DEPT. LA PAZ. Prov. Nor Yungas, near Pongo, $16^{\circ} 19^{\prime} 28^{\prime \prime} \mathrm{S}, 67^{\circ} 57^{\prime} 21^{\prime \prime} \mathrm{W}, 3822 \mathrm{~m}$, Páramo Yungeño, on soil, 13 May 2009, US (LPB, herb. Schiefelbein 3337).

\section{*Cladonia nana Vain.}

In South America this species has been reported from Brazil, Colombia, Ecuador, Paraguay, Peru and Venezuela (Ahti 2000). It is also known from the Azores, the Caribbean, Costa Rica, Guatemala, Mexico, Nicaragua, Panama and southeastern of the U.S.A. (Ahti 2000; Ahti unpubl.)

SPeCimens EXAMINed. BOliVia. Dept. CoCHABAMBA. Prov. Chapare, CARRASCO, $130 \mathrm{~km}$ del camino antiguo de Cochabamba a Villa Tunari, $2200 \mathrm{~m}, 17^{\circ} 07^{\prime} \mathrm{S}, 65^{\circ} 36^{\prime} \mathrm{W}$, bosque húmedo, epiphytic, 10 Oct. 1997, KB et al. 701 (B, LPB); DEPT. LA PAZ. Prov. Bautista Saavedra, $15 \mathrm{~km}$ de Charazani hacia Apolo, $2400 \mathrm{~m}, 15^{\circ} 11^{\prime} \mathrm{S}, 68^{\circ} 52^{\prime} \mathrm{W}$, pastos con arbustos al lado del río Camata, terricolous, 1 July 1997, KB et al. 279 (LPB). ECUADOR. Prov. Tungurahua, río Verde, $1400-1420 \mathrm{~m}, 01^{\circ} 24^{\prime} 03^{\prime \prime} \mathrm{S}, 78^{\circ} 17^{\prime} 43 \mathrm{~W}$, on 
rock, 28 July $1999 \& 24$ Sept. 2000, ZP $2859 \& 4256$ (PRA, QCA).

\section{Cladonia ochrochlora Flörke}

These are the first recently confirmed Bolivian records of this much misinterpreted species (Ahti 2000).

CHEMISTRY. Fumarprotocetraric acid and related substances.

Specimens examined. BOLIVIA. DePt. CochaBAMBA. Prov. Ayopaya, $10 \mathrm{~km}$ de Cocapata hacia Cotacajes, $2800-2850 \mathrm{~m}, 16^{\circ} 38^{\prime} \mathrm{S}, 66^{\circ} 41^{\prime} \mathrm{W}$, bosque húmedo, on decaying wood, 10 May 1997, KB et al. 12 (B, LPB); DEPT. LA PAZ. Prov. Bautista Saavedra, $6 \mathrm{~km}$ de Pauji-Yuyo hacia Charazani, $1350 \mathrm{~m}, 15^{\circ} 02^{\prime} \mathrm{S}$, $68^{\circ} 29^{\prime} \mathrm{W}$, bosque siempreverde, on decaying wood, 14 June 1997, KB et al. 163 (LPB); $15 \mathrm{~km}$ de Charazani hacia Apolo, $2450 \mathrm{~m}, 15^{\circ} 11^{\prime} \mathrm{S}, 68^{\circ} 52^{\prime} \mathrm{W}$, bosque húmedo secundario, at the base of tree, 2 July 1997 , $K B$ et al. 311 (B, LPB); Prov. Larecaja, near Consata, $15^{\circ} 20^{\prime} 46^{\prime \prime} \mathrm{S}, 6^{\circ} 29^{\prime} 16^{\prime \prime} \mathrm{W}, 1240 \mathrm{~m}$, Yungas montano inferior secondary forest, on wood, 15 May 2011, US (LPB, herb. Schiefelbein 3336); Prov. Nor Yungas, Coscapa, senda al oeste del camino principal $c a 4 \mathrm{~km}$ de Cotapata hacia La Paz, $3500 \mathrm{~m}, 16^{\circ} 23^{\prime} \mathrm{S}, 67^{\circ} 53^{\prime} \mathrm{W}$, S-exposicion, bosque siempreverde, epiphytic, 9 Sept. 1997, KB et al. 462 (LPB).

\section{${ }^{*}$ Cladonia ramulosa (With.) J. R. Laundon}

This is a widespread temperate to tropical species; in South America it is already known from Argentina, Brazil, Colombia, Paraguay, Uruguay and Venezuela (Ahti 2000).

CHEMISTRY. Fumarprotocetraric acid and related substances.

Specimens eXamined. BOLIVIA. Dept. La PaZ. Prov. Larecaja, cerca Consata, $15^{\circ} 20^{\prime} 46^{\prime \prime} \mathrm{S}, 68^{\circ} 29^{\prime} 16^{\prime \prime} \mathrm{W}$, $1240 \mathrm{~m}$, Yungas montano inferior secondary forest, on detritus, 15 May 2011, US (LPB, herb. Schiefelbein 2971).

${ }^{*}$ Coenogonium leprieurii (Mont.) Nyl.

This pantropical lichen is known in South America from Brazil, Colombia, French Guiana and Venezuela (Sipman 1992a; Rivas Plata et al. 2006, Cáceres 2007; Sipman et al. 2008).
Specimens examined. BOLIVIA. Dept. LA PAZ. Prov. Nor Yungas, Cotapata cerca a la comunidad del Chairo, $1370 \mathrm{~m}, 16^{\circ} 13^{\prime} 18^{\prime \prime} \mathrm{S}, 67^{\circ} 52^{\prime} 36^{\prime \prime} \mathrm{W}$, manchón de bosque cercano al río Huarinilla, corticolous, 11 May 2006, PR et al. 72 (B, LPB).

\section{Coenogonium linkii Ehrenb.}

In Bolivia this species was reported by Herzog (1922, 1923) and recently by Flakus (2008).

SPECIMENS EXAMINED. BOLIVIA. DEPT. COCHABAMBA. Prov. Chapare, CARRASCO, $130 \mathrm{~km}$ del camino antiguo de Cochabamba a Villa Tunari, $2200 \mathrm{~m}$, $17^{\circ} 07^{\prime} \mathrm{S}, 65^{\circ} 36^{\prime} \mathrm{W}$, bosque húmedo, epiphytic, 10 Oct. 1997, KB et al. 700 (LPB); $137 \mathrm{~km}$ del camino antiguo de Cochabamba a Villa Tunari, $1600 \mathrm{~m}, 17^{\circ} 06^{\prime} \mathrm{S}, 65^{\circ} 35^{\prime} \mathrm{W}$, bosque siempreverde, epiphytic, 11 Oct. 1997, KB et al. 760 (LPB); DEPT. LA PAZ. Prov. Nor Yungas, Hacienda Sacramento, ca $8 \mathrm{~km}$ del camino principal de Chuspipata hacia Coroico, $2500 \mathrm{~m}, 16^{\circ} 24^{\prime} \mathrm{S}, 67^{\circ} 47^{\prime} \mathrm{W}$, bosque siempreverde, epiphytic, 20 Sept. 1997, KB et al. 553 (B, LPB); DePT. SANTA CruZ. Prov. Florida, Refugio Los Volcanes, $1000 \mathrm{~m}, 18^{\circ} 06^{\prime} \mathrm{S}, 63^{\circ} 36^{\prime} \mathrm{W}$, bosque semideciduo, epiphytic, 2 Oct. 1997, KB et al. 596 (LPB).

*Coenogonium nepalense (G. Thor \& Vězda) Lücking, Aptroot \& Sipman

This species is known from Central and South America, and the eastern Paleotropics (Rivas Plata et al. 2006). In South America it has been reported from Argentina and Brazil (Cáceres 2007; Ferraro \& Michlig 2013).

Specimens eXamined. BOliVia. Dept. TariJa. Prov. Burnet O'Connor, road between Entre Ríos and Tarija, $21^{\circ} 28^{\prime} 29^{\prime \prime} \mathrm{S}, 64^{\circ} 12^{\prime} 32^{\prime \prime} \mathrm{W}, 1820 \mathrm{~m}$, TucumanoBoliviano montano forest, on bark, 11 Aug. 2012, MK $11412 a$ (LPB, UGDA).

\section{*Coenogonium pulchrum (Müll. Arg.) Kalb}

Previously this lichen was known only from Brazil and Venezuela (Kalb 1991; Rivas Plata et al. 2006).

SPECIMENS EXAMINED. BOLIVIA. DEPT. COCHABAMBA. Prov. Carrasco, CARRASCO, near Sehuencas, by río López Mendoza, 17³0'26"S, 65¹6'55"W, $2226 \mathrm{~m}$, Yungas cloud forest, corticolous, 22 July 2008, $A F$ 10696, $M K \& P R$ (KRAM, LPB). 


\section{Cora glabrata (Spreng.) Fr.}

Dictyonema glabratum (Spreng.) D. Hawksw.

This species is common in humid habitats in the Bolivian Andes, but few records have been published (Flakus et al. 2012a; Rodriguez Flakus et al. 2013).

Some specimens have granular soredia at the margins of the thalli, but otherwise they match typical material. Although they are tentatively identified as C. glabrata, they require further study.

Specimens examined. BOliVia. Dept. Beni. Prov. José Ballivián, $16 \mathrm{~km}$ por carretera maderera al SW del km 12 Yucumo - Rurrenabaque, $700 \mathrm{~m}$, $15^{\circ} 06^{\prime} \mathrm{S}, 67^{\circ} 07^{\prime} \mathrm{W}$, quebrada con bosque siempreverde, saxicolous, 1 Aug. 1997, KB et al. 370 (B, LPB); DEPT. CochaBAmBA. Prov. Ayopaya, $10 \mathrm{~km}$ de Cocapata hacia Cotacajes, $2750 \mathrm{~m}, 16^{\circ} 38^{\prime} \mathrm{S}, 66^{\circ} 41^{\prime} \mathrm{W}$, bosque húmedo, terricolous, 10 May 1997, KB et al. 1 (B, LPB); Prov. Carrasco, CARRASCO, near Phaqcha, $17^{\circ} 27^{\prime} 13^{\prime \prime} \mathrm{S}$, $65^{\circ} 16^{\prime} 44^{\prime \prime} \mathrm{W}, 2850 \mathrm{~m}$, montane cloud forest, among mosses, 20 July 2008, MK 6237 (LPB, UGDA); near Sehuencas by río López Mendoza, $17^{\circ} 30^{\prime} 26^{\prime \prime} \mathrm{S}$, $65^{\circ} 16^{\prime} 55^{\prime \prime} \mathrm{W}, 2226 \mathrm{~m}$, montane cloud forest, on bark of tree, 22 July 2008, MK 6634 (LPB, UGDA); near Incachaca, $17^{\circ} 15^{\prime} 10^{\prime \prime} \mathrm{S}, 65^{\circ} 48^{\prime} 51^{\prime \prime} \mathrm{W}, 2330 \mathrm{~m}$, disturbed montane cloud forest, on bark of tree, 23 July 2008, MK 6690 (LPB, UGDA); Prov. Chapare, CARRASCO, $132 \mathrm{~km}$ del camino antiguo de Cochabamba a Villa Tunari, $1950 \mathrm{~m}, 17^{\circ} 06^{\prime} \mathrm{S}, 65^{\circ} 35^{\prime} \mathrm{W}$, bosque húmedo, saxicolous, 10 Oct. 1997, KB et al. 673 (B, LPB); Dept. La PAZ. Prov. Camacho, cerca de la comunidad de Ulla Ulla, en el cerro Wilamuku, $4739 \mathrm{~m}$, $15^{\circ} 02.068^{\prime} \mathrm{S}, 69^{\circ} 11.878^{\prime} \mathrm{W}$, vegetación Altoandina, terricolous, 4 Dec. 2007, PR 265 (B, LPB); Prov. Nor Yungas, near Pacallo, $16^{\circ} 12^{\prime} 10^{\prime \prime} \mathrm{S}, 67^{\circ} 50^{\prime} 39^{\prime \prime} \mathrm{W}, 1360 \mathrm{~m}$, montane forest, on bark of tree, 3 Aug. 2008, MK 7140 \& 7166 (LPB, UGDA); between Coroico and La Paz, $16^{\circ} 13^{\prime} 09^{\prime \prime} \mathrm{S}, 67^{\circ} 49^{\prime} 32^{\prime \prime} \mathrm{W}, 2600 \mathrm{~m}$, open place by the road in mountaine cloud forest, on ground, 4 Aug. 2008, MK 7177a \& 7182 (LPB, UGDA); Coroico, Sol y Luna hotel, $1870 \mathrm{~m}, 16^{\circ} 11^{\prime} 56^{\prime \prime} \mathrm{S}, 67^{\circ} 43^{\prime} 26^{\prime \prime} \mathrm{W}$, gardens with trees, on ground, 13 Dec. 2011, MK 10830 (LPB, UGDA); COTAPATA, Santa Catalina, above Tunkini, $16^{\circ} 11^{\prime} 12^{\prime \prime} \mathrm{S}, 67^{\circ} 52^{\prime} 07^{\prime \prime} \mathrm{W}$, ca $2700 \mathrm{~m}$, Yungas montane forest, on ground, 22 May 2011, MK 9059 (LPB, UGDA); Prov. Sud Yungas, Mururata, bajo la mina Bolsa Negra, pasando por el pueblo de Tres Ríos, $3851 \mathrm{~m}, 16^{\circ} 35^{\prime} \mathrm{S}, 67^{\circ} 45^{\prime} \mathrm{W}$, terricolous, 6 Apr. 1995, J. Gonzáles 388 (B, LPB); DEPT. SANTA CRUZ. Prov. Ca- ballero, near Siberia, $17^{\circ} 49^{\prime} 38^{\prime \prime} \mathrm{S}, 64^{\circ} 44^{\prime} 45^{\prime \prime} \mathrm{W}, 3950 \mathrm{~m}$, open Yungas cloud forest, on bark and ground, 16 Aug. 2012, MK 11459 \& 11481 (LPB, UGDA).

SPECIMENS WITH GRANULAR SOREDIA. BOLIVIA. DEPT. COCHABAMBA. Prov. Carrasco, CARRASCO, between Sehuencas and Monte Punku, ca $2650 \mathrm{~m}$, montane cloud forest, on humus, 22 July 2008, $M K 6578$ (LPB); DEPT. LA PAZ. Prov. Nor Yungas, between Coroico and La Paz, $16^{\circ} 13^{\prime} 09^{\prime \prime} \mathrm{S}, 67^{\circ} 49^{\prime} 32^{\prime \prime} \mathrm{W}, 2600 \mathrm{~m}$, open place by the road in montaine cloud forest, on ground, 4 Aug. 2008, MK 7190 (LPB, UGDA); DEPT. SANTA CRUZ. Prov. Caballero, near Siberia, $17^{\circ} 49^{\prime} 38^{\prime \prime} \mathrm{S}, 64^{\circ} 44^{\prime} 45^{\prime \prime} \mathrm{W}$, $3950 \mathrm{~m}$, open Yungas cloud forest, on bark and ground, 16 Aug. 2012, $M K 11462$ (LPB, UGDA).

\section{${ }^{*}$ Crocynia gossypina (Sw.) A. Massal.}

This species is known from Australia, Asia, Oceania, Central and South America (Elix \& McCarthy 1998; Umaña-Tenorio et al. 2002; Feuerer 2013). In South America it has been reported from Brazil, Colombia, French Guiana, Guyana, Surinam and Venezuela (Hekking \& Sipman 1988; Sipman 1992a; Marcano et al. 1996; Cáceres 2007; Sipman et al. 2008; Feuerer 2013). Here is reported as new to Ecuador.

\section{CHEMISTRY. Atranorin and terpenoid.}

Specimens eXAmined. BOLIVIA. DePt. Beni. Prov. Vaca Díez, Cachuela Esperanza, $10^{\circ} 32^{\prime} 09^{\prime \prime} \mathrm{S}$, $65^{\circ} 34^{\prime} 55^{\prime \prime} \mathrm{W}, 128 \mathrm{~m}$, lowland Amazon forest, on granite rock, 4 June 2006, AF 7494, 7497 \& 7498 (KRAM, LPB); DEPT. SANTA CRUZ. Prov. José Miguel de Velasco, sendero de goma near Florida, $14^{\circ} 37^{\prime} 48^{\prime \prime} \mathrm{S}, 61^{\circ} 12^{\prime} 02^{\prime \prime} \mathrm{W}$, $170 \mathrm{~m}$, lowland Amazon secondary forest, corticolous, 16 Dec. 2009, $A F 16128$ \& PR (KRAM, LPB). ECUADOR. Prov. Napo, NP Yasuni, río Tiputini, rainforest, $00^{\circ} 37^{\prime} \mathrm{S}, 76^{\circ} 28^{\prime} \mathrm{W}, 300 \mathrm{~m}$, corticolous, $13 \& 14$. Aug. 1999, ZP $2545 \& 2809$ (PRA, QCA).

\section{Crocynia pyxinoides Nyl.}

Only recently reported from Bolivia (Flakus et al. 2012a).

CHEMISTRY. Atranorin and 1 or 2 terpenoids.

SPeCimens eXamined. BOLIVIA. Dept. La PAZ. Prov. Nor Yungas, Cotapata, a 15 minutos de la carretera Polo Polo, $1375 \mathrm{~m}, 16^{\circ} 10^{\prime} 57.4^{\prime \prime} \mathrm{S}, 67^{\circ} 45^{\prime} 8.4^{\prime \prime} \mathrm{W}$, bosque intervenido cerca encuentra la carretera secundaria hacia 
Charobamba, corticolous, 20 Dec. 2006, PR \& S. Gallegos 336 (B, LPB); DEPT. BENI. Prov. Iténez, Puerto Feliz colony by Río Blanco, $13^{\circ} 09^{\prime} 23^{\prime \prime} \mathrm{S}, 63^{\circ} 43^{\prime} 24^{\prime \prime} \mathrm{W}$, $137 \mathrm{~m}$, lowland Amazon forest, corticolous, 16 Aug. 2008, $A F 12121 \&$ \&R (KRAM); Prov. Vaca Díez, Cachuela Esperanza, $10^{\circ} 32^{\prime} 09^{\prime \prime} \mathrm{S}, 65^{\circ} 34^{\prime} 55^{\prime \prime} \mathrm{W}, 128 \mathrm{~m}$, lowland Amazon forest, on granite rock, 4 June 2006, AF 7493 \& 7495.1 (KRAM, LPB).

\section{* Cryptodiscus similis (Vězda) Baloch}

This rare lichen is known from Brazil and Jamaica (Baloch et al. 2009).

SPECIMENS EXAMINED. BOLIVIA. DEPT. SANTA CRUZ. Prov. Andrés Ibáñez, AMBORÓ, on the road to San Mateo de Toresilla, $17^{\circ} 48^{\prime} 11^{\prime \prime} \mathrm{S}, 64^{\circ} 39^{\prime} 28^{\prime \prime} \mathrm{W}$, $2250 \mathrm{~m}$, Yungas cloud forest, terricolous, 9 June 2011, $A F 22665$ \& $O P$ (KRAM, LPB).

\section{Culbersonia nubila (Moberg) Essl.}

This species has been reported in Bolivia only from two localities (Obermayer et al. 2009).

Specimens examined. Bolivia. Prov. LA PAZ. Prov. Murillo, La Paz, Parque Zenón Iturralde, $3526 \mathrm{~m}, 16^{\circ} 30^{\prime} 24^{\prime \prime} \mathrm{S}, 68^{\circ} 07^{\prime} 12^{\prime \prime} \mathrm{W}$, area verde, 3 July 2008, G. Yapu-Alcázar 221 (B, LPB); DEPT. Potosí. Prov. Nor Lípez, Viscachitas, near del Árbol de Piedra, $21^{\circ} 55^{\prime} 33^{\prime \prime} \mathrm{S}, 68^{\circ} 00^{\prime} 18^{\prime \prime} \mathrm{W}, 4612 \mathrm{~m}$, open semi-desert high Andean area, saxicolous, 5 Dec. 2009, AF 14736.4 $\& P R$ (LPB).

\section{*Dibaeis globulifera Kalb \& Gierl}

This Neotropical lichen is known only from Brazil, Colombia, Costa Rica and Venezuela (Gierl \& Kalb 1993; Umaña-Tenorio et al. 2002; Sipman et al. 2008).

SPECIMENS EXAmined. BOLIVIA. DePt. LA PAZ. Prov. Bautista Saavedra, Cerro Asunta Pata, $1500 \mathrm{~m}$, $15^{\circ} 05^{\prime} \mathrm{S}, 68^{\circ} 29^{\prime} \mathrm{W}$, pared lodosa, saxicolous, 21 June 1997, KB et al. 177 (B, LPB); Prov. Nor Yungas, Serranía Bellavista, $c a 36 \mathrm{~km}$ por camino de Caranavi hacia Sapecho, $1500 \mathrm{~m}, 15^{\circ} 41^{\prime} \mathrm{S}, 67^{\circ} 30^{\prime} \mathrm{W}$, pared rocosa, saxicolous, 31 Aug. 1997, KB et al. 435 (B, LPB).

Dictyonema melvinii Chaves, Lücking \& Umaña

Three localities were so far reported from Bolivia (Chaves et al. 2004; Flakus et al. 2012a).
Specimens examined. BOlivia. Dept. La PaZ. Prov. Bautista Saavedra, Cerro Asunta Pata. 1500 m, $15^{\circ} 05^{\prime} \mathrm{S}, 68^{\circ} 29^{\prime} \mathrm{W}$, bosque siempreverde, epiphytic, 21 June 1997, KB et al. 195 (B, LPB).

*Dictyonema phyllogenum (Müll. Arg.) Zahlbr. s.str.

In the Neotropics this lichen has been reported from Brazil, Costa Rica, Guyana, Ecuador, Panama, Peru and the U.S.A. (Florida) (Lücking 2008; Lücking et al. 2011a).

While this species might be best placed in Cyphellostereum D. A. Reid (Yánez et al. 2012), no formal combination has been made so far.

Specimens eXamined. BOLIVIA. DePt. CochaBAMBA. Prov. Carrasco, CARRASCO, near Phaqcha, $17^{\circ} 27^{\prime} 13^{\prime \prime} \mathrm{S}, 65^{\circ} 16^{\prime} 44^{\prime \prime} \mathrm{W}, 2850 \mathrm{~m}$, montane cloud forest, on bark of tree, 20 July 2008, MK 6206 (LPB, UGDA); Dept. LA Paz. Prov. Bautista Saavedra, Calzada, $15^{\circ} 09^{\prime} 06^{\prime \prime} \mathrm{S}, 68^{\circ} 30^{\prime} 24^{\prime \prime} \mathrm{W}, 835 \mathrm{~m}$, Preandean Amazon secondary forest, on rock, 17 May 2011, MK $8804 a$ (LPB).

Dictyonema schenkianum (Müll. Arg.) Zahlbr.

D. sericeum f. schenkianum (Müll. Arg.) Parm.

Flakus (2008) and Flakus et al. (2012a) recorded this taxon from only three localities in Bolivia.

Specimens examined. BOLIVIA. Dept. CoCHABAMBA. Prov. Carrasco, CARRASCO, Koricaza, $17^{\circ} 33^{\prime} 21^{\prime \prime} \mathrm{S}, 65^{\circ} 16^{\prime} 29^{\prime \prime} \mathrm{W}, 2950 \mathrm{~m}$, Páramo Yungueño, on bark, 18 Aug. 2012, MK 11738 (LPB, UGDA). DEPT. LA PAZ. Prov. Nor Yungas, near Pacallo, $16^{\circ} 12^{\prime} 10^{\prime \prime} \mathrm{S}$, $67^{\circ} 50^{\prime} 39^{\prime \prime} \mathrm{W}, 1360 \mathrm{~m}$, montane forest, on bark of tree, 3 Aug. 2008, $M K$ 7169a (LPB); DEPT. SANTA CRUZ. Prov. Caballero, near Siberia, $17^{\circ} 49^{\prime} 34^{\prime \prime} \mathrm{S}, 64^{\circ} 43^{\prime} 14^{\prime \prime} \mathrm{W}, 2800 \mathrm{~m}$, Yungas cloud forest, on bark, 16 Aug. 2012, MK 11480 (LPB, UGDA); near Siberia, $17^{\circ} 50^{\prime} 15^{\prime \prime} \mathrm{S}, 64^{\circ} 42^{\prime} 36^{\prime \prime} \mathrm{W}$, $2700 \mathrm{~m}$, Yungas cloud forest, on deacying pteridophytes, 16 Aug. 2012, MK 11495 (LPB, UGDA).

Dictyonema sericeum (Sw.) Berk. s.str.

This species has been reported only from a few Bolivian localities by Parmasto (1978) and Flakus (2008).

Specimens examined. BOLIVIA. Dept. CochaBAMBA. Prov. Carrasco, CARRASCO, near Phaqcha, 
$17^{\circ} 27^{\prime} 13^{\prime \prime} \mathrm{S}, 65^{\circ} 16^{\prime} 44^{\prime \prime} \mathrm{W}, 2850 \mathrm{~m}$, montane cloud forest, among mosses, 20 July 2008, MK 6160 (LPB, UGDA); Prov. Chapare, CARRASCO, $130 \mathrm{~km}$ del camino antiguo de Cochabamba a Villa Tunari, $2200 \mathrm{~m}, 17^{\circ} 07^{\prime} \mathrm{S}$, $65^{\circ} 36^{\prime} \mathrm{W}$, bosque húmedo, epiphytic, 10 Oct. 1997, $K B$ et al. 693 (LPB); $137 \mathrm{~km}$ del camino antiguo de Cochabamba a Villa Tunari, $1600 \mathrm{~m}, 17^{\circ} 06^{\prime} \mathrm{S}, 65^{\circ} 35^{\prime} \mathrm{W}$, bosque siempreverde, epiphytic, 11 Oct. 1997, KB et al. 767 (LPB); $139 \mathrm{~km}$ del camino antiguo de Cochabamba a Villa Tunari, $1450 \mathrm{~m}, 17^{\circ} 07^{\prime} \mathrm{S}, 65^{\circ} 34^{\prime} \mathrm{W}$, bosque siempreverde, epiphytic, 12 Oct. $1997, K B$ et al. 798 \& 799 (LPB); $156 \mathrm{~km}$ del camino antiguo de Cochabamba a Villa Tunari, $850 \mathrm{~m}, 17^{\circ} 05^{\prime} \mathrm{S}, 65^{\circ} 32^{\prime} \mathrm{W}$, bosque siempreverde, epiphytic, 12 Oct. 1997, KB et al. 842 (B, LPB); DEPT. LA PAZ. $15 \mathrm{~km}$ de Charazani hacia Apolo, $2450 \mathrm{~m}$, $15^{\circ} 11^{\prime} \mathrm{S}, 68^{\circ} 52^{\prime} \mathrm{W}$, bosque húmedo secundario, at the base of tree, 2 July 1997, KB et al. 318 (B, LPB); $6 \mathrm{~km}$ de Pauji-Yuyo hacia Charazani, $1350 \mathrm{~m}, 15^{\circ} 02^{\prime} \mathrm{S}, 68^{\circ} 29^{\prime} \mathrm{W}$, bosque siempreverde con Iritea y Geonoma, epiphytic, 14 June 1997, KB et al. 159 (LPB); Cerro Asunta Pata, $1500 \mathrm{~m}, 15^{\circ} 05^{\prime} \mathrm{S}, 68^{\circ} 29^{\prime} \mathrm{W}$, bosque siempreverde, epiphytic, 21 June 1997, KB et al. 166 \& 182 (B, LPB); Prov. Nor Yungas, near Pacallo, $16^{\circ} 12^{\prime} 10^{\prime \prime} \mathrm{S}, 6^{\circ} 50^{\prime} 39^{\prime \prime} \mathrm{W}$, $1360 \mathrm{~m}$, montane forest, on bark of tree, 3 Aug. 2008, $M K$ 7169 (LPB, UGDA); Prov. Nor Yungas, Cotapata, 1 hora caminando desde Sandillani hacia Bajo Hornuni, $1520 \mathrm{~m}$, $16^{\circ} 11^{\prime} 06^{\prime \prime} \mathrm{S}, 67^{\circ} 49^{\prime} 30^{\prime \prime} \mathrm{W}$, bosque muy húmedo, corticolous, 13 Oct. 2006, PR et al. 182 (B, LPB); Prov. Larecaja, near Achiquiri, $15^{\circ} 13^{\prime} 31^{\prime \prime} \mathrm{S}, 68^{\circ} 13^{\prime} 49^{\prime \prime} \mathrm{W}, 1070 \mathrm{~m}$, Yungas inferior secondary forest, on bark, 16 May 2011, MK 8654 (LPB, UGDA); Prov. Nor Yungas, COTAPATA, Santa Catalina, above Tunkini, $16^{\circ} 11^{\prime} 12^{\prime \prime} \mathrm{S}, 67^{\circ} 52^{\prime} 07^{\prime \prime} \mathrm{W}$, ca $2700 \mathrm{~m}$, Yungas montane forest, on bark, 22 May 2011, MK 9012 (LPB, UGDA).

\section{Dictyonema sericeum f. phyllophilum Parm.}

This taxon has been reported from only three localities in Bolivia (Flakus et al. 2012a).

It is a member of the $D$. sericeum group and most probably it deserves the rank of species (Chaves et al. 2004; Yánez et al. 2012).

Specimens eXamined. BOLIVIA. DePt. LA PaZ. Prov. Nor Yungas, COTAPATA, by Sillu Tincara preColumbian route, $16^{\circ} 17^{\prime} 22^{\prime \prime} \mathrm{S}, 67^{\circ} 53^{\prime} 29^{\prime \prime} \mathrm{W}, 3520 \mathrm{~m}$, transition Páramo Yungueño-Yungas montane cloud forest, on bark, 25 May 2011, MK 9455 (LPB, UGDA); DEPT. SANTA CRUZ. Prov. Florida, AMBORÓ, above la Yunga, senda Los Helechos, $18^{\circ} 03^{\prime} 30^{\prime \prime} \mathrm{S}, 63^{\circ} 54^{\prime} 36^{\prime \prime} \mathrm{W}$, $2330 \mathrm{~m}$, Yungas cloud forest, on bark, 7 June 2011, MK 9761 (LPB).
*Diploschistella trapperi (Kalb \& Vězda) Lücking, Sérus. \& Vězda

This very rare species is known only from the type locality in Ecuador (Kalb \& Vězda 1988; Lücking et al. 2005).

Specimens eXamined. BOLIVIA. Dept. La PaZ. Prov. Murillo, Valle del Zongo, $16^{\circ} 07^{\prime} 41^{\prime \prime} \mathrm{S}, 68^{\circ} 05^{\prime} 55^{\prime \prime} \mathrm{W}$, $2446 \mathrm{~m}$, Yungas cloud forest, saxicolous, 29 May 2011, $A F 22018$ \& OP (KRAM, LPB).

AdDitional SPECIMEN EXAMINED. Kalb, Lich. Neotrop. 423 (B, isotype of Gyalideopsis trapperi Kalb \& Vězda).

\section{Dirinaria applanata (Fée) D. D. Awasthi}

This pantropical-subtropical species was known from a single locality in Bolivia (Awasthi 1975).

SPECIMENS EXAMINED. BOLIVIA. DEPT. LA PAZ. Prov. Sud Yungas, Alto Beni, Nueva Israel, $15^{\circ} 34^{\prime} 46.2^{\prime \prime} \mathrm{S}$, $67^{\circ} 15^{\prime} 54.6^{\prime \prime} \mathrm{W}, 540 \mathrm{~m}$, plantation, on Theobroma cacao, 25 June 1999, N. Derakshani 14 (B, GOET).

\section{${ }^{*}$ Eschatogonia dissecta Timdal \& R. Sant.}

This lichen is known only from South America (Brazil, French Guiana, Peru, Venezuela) (Timdal 2008a; Kalb et al. 2012).

Specimens eXamined. BOLIVIA. DePt. Beni. Prov. Iténez, San Martínsito colony by río San Martín, $13^{\circ} 18^{\prime} 07^{\prime \prime} \mathrm{S}, 63^{\circ} 25^{\prime} 31^{\prime \prime} \mathrm{W}, 150 \mathrm{~m}$, lowland Amazon forest, corticolous, 20 Aug. 2008, $A F 12753 \& P R$ (KRAM, LPB).

\section{Glyphis cicatricosa Ach.}

This pantropical species was previously reported from Bolivia by Flakus and Wilk (2006).

CHEMISTRY. No substances detected by TLC.

SPecimens eXAmined. BOLIVIA. DePt. Beni. Prov.

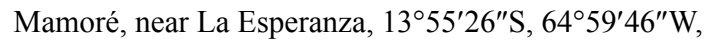
$140 \mathrm{~m}$, los Llanos de Moxos savanna by lake, on bark, 1 Sept. 2012, MK 12013 (LPB, UGDA); Prov. Marbán, near San Lorenzo, $15^{\circ} 12^{\prime} 59^{\prime \prime} \mathrm{S}, 64^{\circ} 47^{\prime} 08^{\prime \prime} \mathrm{W}, 160 \mathrm{~m}$, lowland Amazon forest surrounded by los Llanos de Moxos savanna, on twigs, 28 Aug. 2012, MK 11897 a (UGDA, with a specimen of Glyphis scyphullifera); DEPT. LA PAZ. Prov. Iturralde, near San Pedro, $13^{\circ} 43^{\prime} 59^{\prime \prime}$ S, $68^{\circ} 00^{\prime} 38^{\prime \prime} \mathrm{W}, 250 \mathrm{~m}$, savanna, on bark of tree, 28 July 
2008, MK 6898 (LPB, UGDA); Prov. Bautista Saavedra, on the road between Charazani and Apolo, $15^{\circ} 12^{\prime} 43^{\prime \prime} \mathrm{S}$, $68^{\circ} 47^{\prime} 25^{\prime \prime} \mathrm{W}, 1650 \mathrm{~m}$, Yungas montane forest, open area with boulders, on bark, 18 May 2011, $M K 8868$ (LPB, UGDA); DEPT. SANTA CRUZ. Prov. Cordillera, betweenTucavaca and Roboré, $18^{\circ} 37^{\prime} 56^{\prime \prime} \mathrm{S}, 59^{\circ} 36^{\prime} 50^{\prime \prime} \mathrm{W}, 370 \mathrm{~m}$, transition Chiquitano-Chaqueño forest, on bark, 4 Dec. 2011, MK 10515a (LPB); Prov. Ichilo, AMBORÓ, Macuñucu settlement, $17^{\circ} 43^{\prime} 38^{\prime \prime} \mathrm{S}, 63^{\circ} 35^{\prime} 37^{\prime \prime} \mathrm{W}, 460 \mathrm{~m}$, Amazon forest, on twigs, 6 June 2011, MK 9697 (LPB, UGDA).

\section{Glyphis scyphulifera (Ach.) Staiger}

This lichen is already known from two Bolivian collections (Kukwa et al. 2013).

SPECIMENS EXAMINED. BOLIVIA. DEPT. BENI. Prov.

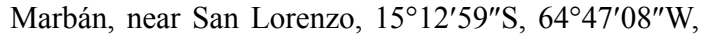
$160 \mathrm{~m}$, lowland Amazon forest surrounded by los Llanos de Moxos savanna, on twigs, 28 Aug. 2012, MK $11897 a$ (LPB, UGDA).

\section{Glyphis substriatula (Nyl.) Staiger}

Kukwa et al. (2013) reported this species from two localities in Bolivia.

SPECIMENS EXAMINED. BOLIVIA. DePt. BENI. Prov. Cercado, Trinidad - Missiones Guarayos, ca $250 \mathrm{~m}$, on bark, Nov. 1926, E. Werdermann 2906 (B); DEPT. SANTA CRUZ. Prov. Cordillera, KAA-IYA, near Peto Blanco, $18^{\circ} 56^{\prime} 26^{\prime \prime} \mathrm{S}, 60^{\circ} 22^{\prime} 39^{\prime \prime} \mathrm{W}, 340 \mathrm{~m}$, Chiquitano forest, on twigs, 5 Dec. 2011, MK 10643 (LPB, UGDA).

Gomphillus hyalinus (Pat.), Lücking, Kalb \& Vězda

This species was recently reported from one locality in Bolivia (Flakus et al. 2012a, as G. ophiosporus Kalb \& Vězda).

Specimens examined. BOLIVIA. Dept. La PaZ. Prov. Bautista Saavedra, $15 \mathrm{~km}$ de Charazani hacia Apolo, $15^{\circ} 11^{\prime} \mathrm{S}, 68^{\circ} 52^{\prime} \mathrm{W}, 3500 \mathrm{~m}$, bosque húmedo, epiphytic, 4 July 1997, KB et al. $342 b$ \& 345 (B, LPB).

\section{Graphis argentata Lücking \& Umaña}

Recently reported from Bolivia by Kukwa et al. (2013).

Chemistry. No lichen compounds detected by TLC.
Specimen eXamined. BOLIVIA. DePt. La PAZ. Prov. Larecaja, near Incachaca, $15^{\circ} 17^{\prime} 48^{\prime \prime} \mathrm{S}, 68^{\circ} 26^{\prime} 32^{\prime \prime} \mathrm{W}$, $1100 \mathrm{~m}$, Yungas montano inferior forest, epiphytic, 15 May 2011, US (LPB).

\section{Graphis duplicata Ach.}

This is the third Bolivian locality for this lichen (Kukwa et al. 2013).

CHEMISTRY. No lichen compounds detected by TLC.

Specimen examined. BOliVia. Dept. La Paz. Prov. Nor Yungas, COTAPATA, between Tunkini and Chairo, $16^{\circ} 11^{\prime} 00^{\prime \prime} \mathrm{S}, 67^{\circ} 52^{\prime} 00^{\prime \prime} \mathrm{W}$, Yungas montane forest, epiphytic, 22 May 2011, US (LPB).

\section{Graphis lumbricina Vain.}

Kukwa et al. (2013) reported one specimen from Bolivia.

\section{CHEMISTRY. Norstictic acid.}

Specimens eXamined. BOLIVIA. Dept. LA PAZ. Prov. Nor Yungas, between Coroico and La Paz, $16^{\circ} 14^{\prime} 30^{\prime \prime} \mathrm{S}, 67^{\circ} 49^{\prime} 58^{\prime \prime} \mathrm{W}, 2848 \mathrm{~m}$, Yungas secondary cloud forest, epiphytic, 23 May 2011, US (LPB, herb. Schiefelbein 3097, 3100, 3101); ibidem, 16 ${ }^{\circ} 13^{\prime} 33^{\prime \prime} \mathrm{S}$, $67^{\circ} 49^{\prime} 20^{\prime \prime} \mathrm{W}, 2600 \mathrm{~m}$, Yungas secondary cloud forest, epiphytic, 23 May 2011, US (LBP, herb. Schiefelbein 3081); Chuspipata, $16^{\circ} 17^{\prime} 09^{\prime \prime} \mathrm{S}, 67^{\circ} 51^{\prime} 00^{\prime \prime} \mathrm{W}, 3228 \mathrm{~m}$, Yungas cloud forest, epiphytic, 24 May 2011, US (LPB).

\section{Graphis nudiformis Lücking}

This species was recently reported from Bolivia, that being the first record from the Southern Hemisphere (Kukwa et al. 2013).

Chemistry. No lichen compounds detected by TLC.

Specimen eXamined. BOLIVIA. Dept. La PaZ. Prov. Nor Yungas, near Nogalani, on the road from Coroico to La Paz, 16 ${ }^{\circ} 12^{\prime} 57^{\prime \prime} \mathrm{S}, 67^{\circ} 49^{\prime} 15^{\prime \prime} \mathrm{W}, 2168 \mathrm{~m}$, Yungas secondary cloud forest, epiphytic, 23 May 2011, US (LPB).

\section{Graphis pinicola Zahlbr.}

The first South American record was recently reported from Bolivia by Kukwa et al. (2013). 
Chemistry. No lichen compounds detected by TLC.

SPECIMENS EXAMINED. BOLIVIA. DEPT. LA PAZ. Prov. Franz Tamayo, near Apolo, $14^{\circ} 50^{\prime} 15^{\prime \prime}$ S, $68^{\circ} 26^{\prime} 58^{\prime \prime} \mathrm{W}, 1430 \mathrm{~m}$, Preandean Amazon forest, epiphytic, 17 May 2011, US (LPB, herb. Schiefelbein 2993); Prov. Larecaja, near Charapampa, $15^{\circ} 15^{\prime} 22^{\prime \prime} \mathrm{S}$, $68^{\circ} 13^{\prime} 02^{\prime \prime} \mathrm{W}, 760 \mathrm{~m}$, Amazon secondary forest, epiphytic, 16 May 2011, US (LPB, herb. Schiefelbein 2984); Prov. Nor Yungas, COTAPATA, Santa Catalina, above Tunkini, $16^{\circ} 11^{\prime} 12^{\prime \prime} \mathrm{S}, 67^{\circ} 52^{\prime} 07^{\prime \prime} \mathrm{W}$, Yungas montane forest, epiphytic, 22 May 2011, US (LPB, herb. Schiefelbein 3028).

Graphis ruiziana (Fée) A. Massal.

Two localities were recently reported from Bolivia (Kukwa et al. 2013).

CHEMISTRY. No lichen compounds detected by TLC.

SPeCiMENS EXAMINED. BOLIVIA. DePt. LA PAZ. Prov. Nor Yungas, COTAPATA, by Sillu Tincara pre-Columbian route, $16^{\circ} 17^{\prime} 22^{\prime \prime} \mathrm{S}, 67^{\circ} 53^{\prime} 29^{\prime \prime} \mathrm{W}, 3518 \mathrm{~m}$, transition Páramo Yungueño-Yungas montane cloud forest, epiphytic, 25 May 2011, US (LPB, herb. Schiefelbein 3454).

\section{Graphis subflexibilis Lücking \& Chaves}

This species was recorded for the first time from Bolivia and the Southern Hemisphere by Kukwa et al. (2013).

Chemistry. No lichen compounds detected by TLC.

SPECIMENS EXAMINED. BOLIVIA. DePt. LA PAZ. Prov. Larecaja, near Achiquiri, $18 \mathrm{~km}$ from Mapiri on the road to Apolo, $15^{\circ} 13^{\prime} 31^{\prime \prime} \mathrm{S}, 68^{\circ} 13^{\prime} 49^{\prime \prime} \mathrm{W}, 1070 \mathrm{~m}$, Yungas inferior secondary forest, epiphytic, 16 May 2011, US (LPB); Prov. Nor Yungas, COTAPATA, Chairo, $16^{\circ} 12^{\prime} 07^{\prime \prime} \mathrm{S}, 67^{\circ} 50^{\prime} 05^{\prime \prime} \mathrm{W}, 1300 \mathrm{~m}$, Yungas montano inferior forest, epiphytic, 21 May 2011, US (LPB); Prov. Nor Yungas, between Coroico and La Paz, $16^{\circ} 13^{\prime} 33^{\prime \prime} \mathrm{S}$, $67^{\circ} 49^{\prime} 20^{\prime \prime} \mathrm{W}, 2600 \mathrm{~m}$, Yungas secondary cloud forest, epiphytic, 23 May 2011, US (LPB).

\section{Graphis tumidula (Fée) Spreng.}

This rare Neotropical lichen has been reported from two localities in Bolivia by Kukwa et al. (2013).
Chemistry. No lichen compounds detected by TLC.

Specimen examined. BOLIVIA. Dept. La PaZ. Prov. Nor Yungas, COTAPATA, Santa Catalina, above Tunkini, $16^{\circ} 11^{\prime} 12^{\prime \prime} \mathrm{S}, 67^{\circ} 52^{\prime} 07^{\prime \prime} \mathrm{W}$, Yungas montane forest, epiphytic, 22 May 2011, US (LPB).

\section{*Gyalidea hyalinescens (Nyl.) Vězda}

In the Neotropics this species has been reported from Costa Rica and Ecuador (Umaña-Tenorio et al. 2002; Nöske \& Sipman 2004); it is also known from Europe, Australia and New Zealand (e.g., Aptroot et al. 1999; Coppins 2002; Feuerer 2013; McCarthy 2013).

Specimen examined. BOLIVIA. DePt. CoChabAMBA. Prov. Chapare, CARRASCO , $123 \mathrm{~km}$ del camino antiguo de Cochabamba a Villa Tunari, $2100 \mathrm{~m}, 17^{\circ} 06^{\prime} \mathrm{S}, 65^{\circ} 37^{\prime} \mathrm{W}$, borde de camino con árbustos y paredes rocosas, saxicolous, 10 Oct. 1997, KB et al. 745 (LPB).

\section{*Gyalideopsis kalbii Vězda}

So far known only from the type locality in Brazil (Vězda 1983).

Specimen examined. BOliVia. Dept. Santa CRUZ. Prov. Andrés Ibáñez, AMBORÓ, on the road to San Mateo de Toresilla, $17^{\circ} 48^{\prime} 11^{\prime \prime} \mathrm{S}, 64^{\circ} 39^{\prime} 28^{\prime \prime} \mathrm{W}$, $2250 \mathrm{~m}$, Yungas cloud forest, terricolous, 9 June 2011, $A F 22665.1 \& O P$ (KRAM, LPB).

Additional SPECIMEN EXAMINED. Vězda, Lich. Sel. Exs. 1965 (B, isotype).

\section{*Heterocyphelium leucampyx (Tuck.) Vain.}

This tropical lichen is known from Africa, Australia and the Neotropics (from Florida to Venezuela and Brazil) (Tibell 1996).

The species produces 2-septate ascospores (Tibell 1996), but one specimen, cited below as $H$. aff. leucampyx, has 3-septate ascospores and requires further study.

SPECIMENS EXAMINED. BOLIVIA. DEPT. TARIJA. Prov. Aniceto Arce, Filo de Sidras, $22^{\circ} 14^{\prime} 50^{\prime \prime} \mathrm{S}$, $64^{\circ} 33^{\prime} 28^{\prime \prime} \mathrm{W}, 1064 \mathrm{~m}$, Tucumano-Boliviano submontane forest, on bark, 22 Nov. 2010, $A F 18390$ (KRAM, LPB); TARIQUÍA, La Hierba, $22^{\circ} 08^{\prime} 46^{\prime \prime} \mathrm{S}, 64^{\circ} 31^{\prime} 36^{\prime \prime} \mathrm{W}$, 
$1070 \mathrm{~m}$, Tucumano-Boliviano submontane forest, near river, on bark, 25 Nov. 2010, $A F 19700 \& J Q$ (KRAM, LPB).

SPECIMEN OF HETEROCYPHELIUM AFF. LEUCAMPYX. BOliviA. Dept. Santa Cruz. Prov. José Miguel de Velasco, sendero de goma, near Florida, $14^{\circ} 37^{\prime} 48^{\prime \prime} \mathrm{S}$, $61^{\circ} 12^{\prime} 02^{\prime \prime} \mathrm{W}, 170 \mathrm{~m}$, lowland Amazon secondary forest, 16 Dec. 2009, on bark, $A F 16082.1 \& P R$ (LPB)

\section{${ }^{*}$ Heterodermia arvidssonii Moberg}

This species was recently described from Ecuador (Moberg 2011).

Specimens eXamined. BOLIVIA. Dept. LA PAZ. Prov. Bautista Saavedra, $15 \mathrm{~km}$ from Charazani, $15^{\circ} 11^{\prime} 02^{\prime \prime} \mathrm{S}, 68^{\circ} 53^{\prime} 43^{\prime \prime} \mathrm{W}, 2560 \mathrm{~m}$, transition Yungas montane forest-cloud forest, on twigs, 18 May 2011, MK $8953 a$ (LPB); DEPT. TARIJA. Prov. O'Connor, near San Diego, $21^{\circ} 26^{\prime} 28^{\prime \prime} \mathrm{S}, 64^{\circ} 14^{\prime} 37^{\prime \prime} \mathrm{W}, 1620 \mathrm{~m}$, TucumanoBoliviano altimontano forest, on bark, 9 Aug. 2012, $M K$ 11187 (LPB, UGDA).

\section{${ }^{*}$ Heterodermia diademata (Tayl.) D. D. Awasthi}

This lichen has been reported from Argentina, Brazil, Ecuador, Paraguay, Peru and Uruguay, and elsewhere from Africa, Asia, Australia and Central America (Moberg 2011).

SPECIMENS EXAMINED. BOLIVIA. DEPT. BENI. Prov. José Ballivián, $20 \mathrm{~km}$ por carretera maderera al SW del km 12 Yucumo - Rurrenabaque, 900-950 m, 1507'S, $67^{\circ} 08^{\prime} \mathrm{W}$, bosque siempreverde, epiphytic, 6 Aug. 1997, $K B$ et al. 386 (B, LPB); DePT. COCHABAmBA. Prov. Chapare, CARRASCO, $139 \mathrm{~km}$ del camino antiguo de Cochabamba a Villa Tunari, $1450 \mathrm{~m}, 17^{\circ} 07^{\prime} \mathrm{S}, 65^{\circ} 34^{\prime} \mathrm{W}$, bosque siempreverde, epiphytic, 12 Oct. 1997, KB et al. 800 (LPB).

\section{Heterodermia flabellata (Fée) D. D. Awasthi}

Prior to this only two ollections of this species were known from Bolivia (Flakus et al. 2012a).

CHEMISTRY. Atranorin, zeorin and leucotylin (selected specimens examined by TLC); pigment on the undersurface of apothecial lobules $\mathrm{K}+$ violet.

Specimens examined. BOlivia. Dept. CoChabAMBA. Prov. Ayopaya, $20 \mathrm{~km}$ de Cocapata hacia Cotacajes, $1850-2000 \mathrm{~m}, 16^{\circ} 46^{\prime} \mathrm{S}, 66^{\circ} 44^{\prime} \mathrm{W}$, bosque semideciduo intervenido, epiphytic, 15 May 1997, KB et al. 86 (LPB); Prov. Carrasco, CARRASCO, near Sehuencas, $17^{\circ} 30^{\prime} 12^{\prime \prime} \mathrm{S}, 65^{\circ} 16^{\prime} 30^{\prime \prime} \mathrm{W}, 2220 \mathrm{~m}$, montane cloud forest, along the road and close to the settlement, on bark of tree, 21 July 2008, MK 6320 (LPB, UGDA); DEPT. LA PAZ. Prov. Bautista Saavedra, $15 \mathrm{~km}$ de Charazani hacia Apolo, $2400 \mathrm{~m}, 16^{\circ} 11^{\prime} 06^{\prime \prime} \mathrm{S}, 67^{\circ} 49^{\prime} 30^{\prime \prime} \mathrm{W}$, bosque humedo secundario con rocas, epiphytic, 2 July 1997, KB et al. 301a (LPB); ibidem, astos con arbustos al lado del Río Camata, terricolous, 1 July 1997, KB et al. $261 c$ \& $273 a$ (B, LPB); Prov. Iturralde, $46 \mathrm{~km}$ por camino de Ixiamas hacia Alto Madidi, $300 \mathrm{~m}$, $13^{\circ} 38^{\prime} \mathrm{S}, 68^{\circ} 26^{\prime} \mathrm{W}$, bosque siempreverde, epiphytic, 13 Aug. 1997, KB et al. 395 (LPB); Prov. Nor Yungas, Cotapata, $1520 \mathrm{~m}, 16^{\circ} 11^{\prime} 06^{\prime \prime} \mathrm{S}, 67^{\circ} 49^{\prime} 30^{\prime \prime} \mathrm{W}$, bosque muy húmedo, corticolous, 13 Oct. 2006, PR et al. 135 (B, LPB).

Heterodermia isidiophora (Vain.) D. D. Awasthi

This species has been reported from Bolivia by Moberg (2011).

CHEMISTRY. Atranorin, zeorin and additional terpenoids (one specimen tested by TLC).

SPECIMENS EXAMINED. BOLIVIA. DEPT. COCHABAMBA. Prov. Ayopaya, $10 \mathrm{~km}$ de Cocapata hacia Cotacajes, $2750 \mathrm{~m}, 16^{\circ} 38^{\prime} \mathrm{S}, 66^{\circ} 41^{\prime} \mathrm{W}$, bosque húmedo, on decaying wood, 12 May 1997, KB et al. 51 (B, LPB, with a specimen of Heterodermia corallophora); Prov. Carrasco, CARRASCO, near Sehuencas by río López Mendoza, $17^{\circ} 30^{\prime} 26^{\prime \prime} \mathrm{S}, 65^{\circ} 16^{\prime} 55^{\prime \prime} \mathrm{W}, 2226 \mathrm{~m}$, montane cloud forest, on bark, 22 July 2008, MK 6642 (LPB, UGDA).

Heterodermia japonica (M. Satô) Swinscow \& Krog

Only a few collections have been reported from Bolivia (Flakus et al. 2012a).

Chemistry. Not tested by TLC; the thallus did not react with $\mathrm{K}$.

SPeCimens eXamined. BOLIVIA. Dept. La PAZ. Prov. Franz Tamayo, between Apolo and Mapiri, $14^{\circ} 41^{\prime} 50^{\prime \prime} \mathrm{S}, 68^{\circ} 25^{\prime} 07^{\prime \prime} \mathrm{W}, 1510 \mathrm{~m}$, savannah with scattered trees, on soil, 22 Nov. 2011, MK 10166 (LPB, UGDA).

\section{Heterodermia palpebrata (Tayl.) Vain.}

Flakus et al. (2012a) recorded this species from three localities in Bolivia. 
Specimens eXamined. BOLIVIA. DePt. LA PAZ. Prov. Bautista Saavedra, near Charazani, $15^{\circ} 10^{\prime} 39^{\prime \prime} \mathrm{S}$, $68^{\circ} 56^{\prime} 36^{\prime \prime} \mathrm{W}, 2730 \mathrm{~m}$, Yungas montane forest, on twigs, 18 May 2011, $M K 8824$ (LPB, UGDA). Prov. Franz Tamayo, between Apolo and Mapiri, $14^{\circ} 41^{\prime} 50^{\prime \prime} \mathrm{S}$, $68^{\circ} 25^{\prime} 07^{\prime \prime} \mathrm{W}, 1510 \mathrm{~m}$, savannah with scattered trees, on bark, 22 Nov. 2011, MK 10237 (LPB).

\section{Heterodermia reagens (Kurok.) Elix}

H. japonica var. reagens (Kurok.) J. N. Wu \& Z. G. Qian.

Reported here for the second time from Bolivia (Flakus et al. 2012a).

CHEMISTRY. Atranorin, zeorin, norstictic acid and fatty acid.

SPeCimens eXAmined. BOLIVIA. DePt. CochaBAMBA. Prov. Carrasco, CARRASCO, near Sehuencas, $17^{\circ} 30^{\prime} 12^{\prime \prime} \mathrm{S}, 65^{\circ} 16^{\prime} 30^{\prime \prime} \mathrm{W}, 2220 \mathrm{~m}$, montane cloud forest, on bark of tree, 21 July $2008, M K 6458 \& 6351 b$ (LPB, UGDA).

\section{${ }^{*}$ Heterodermia spinigera (Kurok.) Moberg}

Previously this species was known only from Ecuador and Peru (Moberg 2011).

Specimens examined. BOlivia. Dept. CoCHABAMBA. Prov. Ayopaya, $20 \mathrm{~km}$ de Cocapata hacia Cotacajes, $1850-2000 \mathrm{~m}, 16^{\circ} 46^{\prime} \mathrm{S}, 66^{\circ} 44^{\prime} \mathrm{W}$, bosque semideciduo intervenido, epiphytic, 15 May 1997, KB et al. 85 \& 94 (B, LPB); DEPT. LA PAZ. Prov. Nor Yungas, COTAPATA, between Tunkini and Chairo, above Tunkini, $16^{\circ} 11^{\prime} \mathrm{S}, 67^{\circ} 52^{\prime} \mathrm{W}, 2400 \mathrm{~m}$, on bark, 22 May 2011, MK 9118 a (LPB, UGDA); DEPT. SANTA Cruz. Prov. Florida, Refugio Los Volcanes, $1000 \mathrm{~m}$, $18^{\circ} 06^{\prime} \mathrm{S}, 63^{\circ} 36^{\prime} \mathrm{W}$, bosque semi-deciduo, epiphytic, 2 Oct. 1997, KB et al. 605 (LPB).

\section{${ }^{*}$ Heterodermia squamulosa Degel.}

This species occurs in Argentina, Brazil, Ecuador, Peru and Venezuela (Moberg 2011); it is also known from Asia, North America and Macaronesia (Madeira) (Sipman 1993; Hafellner 1995; Moberg 2011).

CHEMISTRY. Atranorin, zeorin and two additional terpenoids.

SPECIMENS EXAMINED. BOLIVIA. DEPT. COCHA-
BAMBA. Prov. Carrasco, CARRASCO, near Phaqcha, $17^{\circ} 27^{\prime} 13^{\prime \prime} \mathrm{S}, 65^{\circ} 16^{\prime} 44^{\prime \prime} \mathrm{W}, 2850 \mathrm{~m}$, montane cloud forest, close to the river, on bark of tree, 20 July 2008 , MK 6247a (LPB, UGDA); near Sehuencas, $17^{\circ} 30^{\prime} 12^{\prime \prime} \mathrm{S}$, $65^{\circ} 16^{\prime} 30^{\prime \prime} \mathrm{W}, 2220 \mathrm{~m}$, montane cloud forest, with some rocks in open place, on bark of tree, 21 July 2008, MK 6547 (LPB, UGDA); Prov. Chapare, CARRASCO, $137 \mathrm{~km}$ del camino antiguo de Cochabamba a Villa Tunari, $1600 \mathrm{~m}, 17^{\circ} 06^{\prime} \mathrm{S}, 65^{\circ} 35^{\prime} \mathrm{W}$, bosque siempreverde, epiphytic, 11 Oct. 1997, KB et al. 772 (LPB); DEPT. LA PAZ. Prov. Franz Tamayo, río Bilipisa, $c a 10 \mathrm{~km}$ NW de Apolo, $1100 \mathrm{~m}, 14^{\circ} 36^{\prime} \mathrm{S}, 68^{\circ} 27^{\prime} \mathrm{W}$, bosque semideciduo intervenido, epífita, 4 July 1997, KB 241 (LPB); Prov. Nor Yungas, Cotapata cerca a la comunidad del Chairo, $1370 \mathrm{~m}, 16^{\circ} 13^{\prime} 18^{\prime \prime} \mathrm{S}, 67^{\circ} 52^{\prime} 36^{\prime \prime} \mathrm{W}$, manchón de bosque cercano, corticolous, 11 May, 2006, PR et al. 79 (B, LPB); Cotapata, 1 hora caminando desde Sandillani hacia Bajo Hornuni, $1520 \mathrm{~m}, 16^{\circ} 11^{\prime} 06^{\prime \prime} \mathrm{S}, 67^{\circ} 49^{\prime} 30^{\prime \prime} \mathrm{W}$, bosque muy húmedo, corticolous, 13 Oct. 2006, $P R$ et al. 136 (B, LPB).

\section{${ }^{*}$ Hyperphyscia syncolla (Tuck.) Kalb}

This species has been reported from Argentina, Brazil, Columbia, Peru and Uruguay (Osorio 1992; Rambold et al. 1994; Calvelo \& Liberatore 2002; Spielmann 2006; Sipman et al. 2008); it is also known from Africa, Asia and North America (Feuerer 2013).

Specimens examined. BOlivia. DePt. CoCHABAMBA. Prov. Cercado, Cochabamba, $2500 \mathrm{~m}$, $17^{\circ} 22^{\prime} 00^{\prime \prime} \mathrm{S}, 6^{\circ} 08^{\prime} 30^{\prime \prime} \mathrm{W}$, vegetación de los Valles Secos Interandinos, on Jacaranda mimosifolia, 27 Sept. 2006, M. Canazas MC-73 (B, BOLV); ibidem, $17^{\circ} 23^{\prime} \mathrm{S}$, $66^{\circ} 08.6^{\prime} \mathrm{W}$, on Grevillea robusta, M. Canazas MC-80 (B, BOLV); ibidem, $17^{\circ} 22^{\prime} 00^{\prime \prime} \mathrm{S}, 66^{\circ} 08^{\prime} 30^{\prime \prime} \mathrm{W}$, on Jacaranda mimosifolia, M. Canazas MC-81 (B, BOLV); ibidem, on J. mimosifolia, M. Canazas MC-83 (B, BOLV); ibidem, $17^{\circ} 28^{\prime} 12^{\prime \prime} \mathrm{S}, 66^{\circ} 08^{\prime} 36^{\prime \prime} \mathrm{W}$, on Grevillea robusta, $\mathrm{M}$. Canazas $M C-84 \& M C-85$ (B, BOLV).

*Ingvariella bispora (Bagl.) Guderley \& Lumbsch

This widely distributed species is known from every continent except Antarctica (e.g., Lumbsch 1989; Hafellner 1995; Hafellner \& Türk 2001; Breuss \& John 2004). In South America it has been reported from Argentina and Uruguay (Lumbsch 1989; Osorio 2000).

CHEMISTRY. No substances detected by TLC. 
Specimens examined. BOLIVIA. Dept. LA PAZ. Prov. Omasuyos, El Dragon hill by Chahualla, $15^{\circ} 51^{\prime} 17^{\prime \prime} \mathrm{S}, 69^{\circ} 00^{\prime} 40^{\prime \prime} \mathrm{W}, 3850 \mathrm{~m}$, Puna Húmeda, saxicolous, 6 July 2010, AF 17832 \& PR (LPB); DEPT. SANTA CRUZ. Prov. Manuel María Caballero, Siberia, $17^{\circ} 49^{\prime} 38^{\prime \prime} \mathrm{S}, 64^{\circ} 45^{\prime} 14^{\prime \prime} \mathrm{W}, 3000 \mathrm{~m}$, open area near Yungas cloud forest, saxicolous, 15 Dec. 2004, AF 4827 (KRAM).

\section{Lecanora farinacea Fée}

This species has been reported from Bolivia (as L. blanda Nyl.) only by Nylander (1861).

Specimens examined. BOLIVIA. Dept. LA PaZ. Prov. Murillo, camino a la Cumbre, $4850 \mathrm{~m}, \mathrm{ca} 16^{\circ} 0^{\prime} \mathrm{S}$, $68^{\circ} 0^{\prime} \mathrm{W}$, piso subnival, vegetación Altoandina, on rock, 8 Feb. 2002, A. D. Canseco-Tarifa \& J. L. Vila 148 (B, LPB).

\section{Lecanora stenotropa Nyl.}

Recently reported from Bolivia by Śliwa et al. (2012).

Specimens examined. BOLIVIA. Dept. LA PAZ. Prov. Camacho, cerca de la comunidad de Ulla Ulla, en el cerro Wilamuku, $4770 \mathrm{~m}, 15^{\circ} 01^{\prime} 36.90^{\prime \prime} \mathrm{S}$, $69^{\circ} 11^{\prime} 52.9^{\prime \prime} \mathrm{W}$, vegetación Altoandina, on rock, 4 Dec. 2007, PR 245 (B, LPB).

\section{${ }^{* * *}$ Lepraria elobata Tønsberg}

This species was previously known mainly from numerous European records, less commonly from Asia and North America (e.g., Tønsberg 1992, 1998; Czyżewska \& Kukwa 2005; Saag et al. 2009; Urbanavichus 2010).

CHEMISTRY. Atranorin, zeorin and the stictic acid complex.

Specimens eXamined. BOLIVIA. Dept. La PaZ. Prov. Nor Yungas, COTAPATA, by Sillu Tincara preColumbian route, $16^{\circ} 17^{\prime} 38^{\prime \prime} \mathrm{S}, 6^{\circ} 53^{\prime} 33^{\prime \prime} \mathrm{W}, 3437 \mathrm{~m}$, Yungas cloud forest, on bryophytes, 23 Dec. 2009, $A F$ $16350 \& P R$ (LPB).

\section{${ }^{* *}$ Lepraria jackii Tønsberg}

This widespread species was previously known from Europe, North America, Asia and Australia (Tønsberg 1992; Elix 2009a; Saag et al. 2009; Urbanavichus 2010).
ChEMISTRY. Atranorin, jackinic and roccellic acids.

Specimens examined. BOLIVIA. Dept. La Paz. Prov. Nor Yungas, COTAPATA, by Sillu Tincara preColumbian route, $16^{\circ} 17^{\prime} 38^{\prime \prime} \mathrm{S}, 67^{\circ} 53^{\prime} 33^{\prime \prime} \mathrm{W}, 3437 \mathrm{~m}$, Yungas cloud forest, on soil and bryophytes, 23 Dec. 2009, AF 16323 \& PR (KRAM, LPB).

\section{Lepraria lecanorica Tønsberg}

This is the second collection of this lichen from Bolivia (Flakus \& Kukwa 2007)

CHEMISTRY. Atranorin and lecanoric acid.

SPECIMENS EXAMINED. BOLIVIA. DEPT. LA PAZ. Prov. Franz Tamayo, APOLOBAMBA, near Puyo Puyo, $14^{\circ} 56^{\prime} 55^{\prime \prime} \mathrm{S}, 6^{\circ} 07^{\prime} 58^{\prime \prime} \mathrm{W}, 4795 \mathrm{~m}$, high Andean open vegetation, on schist rock, 5 July 2010, $A F 17680 \& P R$ (KRAM, LPB).

Leprocaulon aff. textum (K. Knudsen, Elix \& Lendemer) Lendemer \& Hodkinson

This species was mentioned briefly by Flakus et al. (2011b). The Bolivian material differs from North American specimens by the absence of atranorin and fatty acids (Knudsen \& Elix 2007); at present it is not clear whether this should be considered infraspecific variation.

CHEMISTRY. Usnic acid and zeorin $( \pm)$.

SPECIMENS EXAMINED. BOLIVIA. DEPT. ORURo. Prov. Sajama, SAJAMA, Jecha K'ala, $18^{\circ} 09^{\prime} 52^{\prime \prime}$, $68^{\circ} 49^{\prime} 08^{\prime \prime} \mathrm{W}, 4184 \mathrm{~m}$, Puna Sureña, grassland vegetation, on soil and bryophytes, 20 June 2010, AF 16703 \& PR (LPB); DEPT. POTOSí. Prov. Nor Lípez, Viscachitas, near del Árbol de Piedra, 21 ${ }^{\circ} 55^{\prime} 33^{\prime \prime} \mathrm{S}, 68^{\circ} 00^{\prime} 18^{\prime \prime} \mathrm{W}$, $4612 \mathrm{~m}$, open semi-desert high Andean area, on soil and bryophytes, 5 Dec. 2009, AF 14736.3, 14736.17, $14736.18 \& P R$ (KRAM, LPB).

\section{${ }^{*}$ Letrouitia flavidula (Tuck.) Hafellner}

This species is already known from Argentina, Brazil, Colombia, Costa Rica, Cuba, Mexico and Puerto Rico (Nylander 1863; Hafellner 1983; Breuss 2001; Calvelo \& Liberatore 2002, Sipman et al. 2008).

Specimens eXamined. BOLIVIA. DePt. La PaZ. Prov. Nor Yungas, Cotapata, $1520 \mathrm{~m}, 16^{\circ} 11^{\prime} 06^{\prime \prime} \mathrm{S}$, 
$67^{\circ} 49^{\prime} 30^{\prime \prime} \mathrm{W}$, bosque muy húmedo, corticolous, 13 Oct. 2006, PR et al. 150 (B, LPB).

*Malmidea amazonica (Redinger) Kalb, Rivas Plata \& Lumbsch

This Neotropical lichen has been reported from Brazil, Costa Rica, French Guiana and Guyana (Redinger 1933; Breuss \& Neuwirth 2007; Lücking 2008).

Specimens eXAmined. BOLIVIA. DePt. Beni. Prov. Iténez, Chaco lejos area, near Bella Vista, $13^{\circ} 16^{\prime} 24^{\prime \prime} \mathrm{S}, 63^{\circ} 42^{\prime} 26^{\prime \prime} \mathrm{W}, 140 \mathrm{~m}$, lowland Amazon forest, corticolous, 17 Aug. 2008, $A F 12388 \& P R$ (KRAM, LPB); DEPT. SANTA CRUZ. Prov. José Miguel de Velasco, PN Noel Kempff Mercado, camino a la Meseta, near Florida, $14^{\circ} 33^{\prime} 31^{\prime \prime} \mathrm{S}, 61^{\circ} 48^{\prime} 08^{\prime \prime} \mathrm{W}, 220 \mathrm{~m}$, lowland Amazon forest, corticolous, 15 Dec. 2009, $A F$ $16035 \& P R$ (LPB).

*Malmidea granifera (Ach.) Kalb, Rivas Plata \& Lumbsch

A pantropical species, its range includes Brazil, Costa Rica, Colombia, Ecuador and Panama (Breuss \& Neuwirth 2007; Cáceres 2007; Breuss 2008; Lücking 2008; Sipman et al. 2008).

Specimens examined. BOlivia. Dept. Beni. Prov. Iténez, El Prado colony, near Bella Vista, by río San Martín, $13^{\circ} 17^{\prime} 46^{\prime \prime} \mathrm{S}, 63^{\circ} 35^{\prime} 21^{\prime \prime} \mathrm{W}, 155 \mathrm{~m}$, lowland Amazon forest, corticolous, 15 Aug. 2008, $A F$ $12011.1 \& P R$ (KRAM, LPB); Chaco lejos area, near Bella Vista, $13^{\circ} 16^{\prime} 24^{\prime \prime} \mathrm{S}, 63^{\circ} 42^{\prime} 26^{\prime \prime} \mathrm{W}, 140 \mathrm{~m}$, lowland Amazon forest, corticolous, 17 Aug. 2008, AF 12402 \& $P R$ (KRAM, LPB).

\section{Malmidea hypomelaena (Nyl.) Kalb \& Lücking}

This is the first modern Bolivian record of this species. It was previously reported from the country by Nylander (1859).

SPECIMEN EXAMINED. BOLIVIA. DePt. TARIJA. Prov. Aniceto Arce, Filo de Sidras, near campamento de guardaparques, $22^{\circ} 14^{\prime} 50^{\prime \prime} \mathrm{S}, 64^{\circ} 33^{\prime} 28^{\prime \prime} \mathrm{W}, 1064 \mathrm{~m}$, Tucumano-Boliviano submontane forest, corticolous, 22 Nov. 2010, $A F 18507$ (LPB).

Malmidea leptoloma (Müll. Arg.) Kalb \& Lücking

This species was reported from Bolivia only by Müller (1895).
Specimens examined. BOLIVIA. Dept. TARIJA. Prov. Aniceto Arce, Filo de Sidras, $22^{\circ} 14^{\prime} 50^{\prime \prime}$ S, $64^{\circ} 33^{\prime} 28^{\prime \prime} \mathrm{W}, 1064 \mathrm{~m}$, Tucumano-Boliviano submontane forest, corticolous, 22 Nov. 2010, AF 18680 (KRAM, LPB).

Malmidea piperis (Spreng.) Kalb, Rivas Plata \& Lumbsch

Reported from Bolivia only by Nylander (1859) and Flakus (2008).

Specimens examined. BOLIVIA. Dept. Santa Cruz. Prov. José Miguel de Velasco, PN Noel Kempff

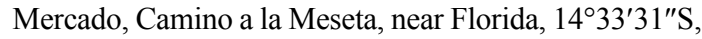
$61^{\circ} 48^{\prime} 08^{\prime \prime} \mathrm{W}, 220 \mathrm{~m}$, lowland Amazon forest, corticolous, 15 Dec. 2009, $A F$ 16035.2 \& PR (KRAM, LPB).

Malmidea rhodopsis (Tuck.) Kalb, Rivas Plata \& Lumbsch

This species has been reported from two Bolivian localities by Flakus (2008).

Specimens EXAmined. BOLIVIA. Dept. LA PAZ. Prov. Abel Iturralde, near San Pedro, $13^{\circ} 44^{\prime} 35^{\prime \prime} \mathrm{S}$, $68^{\circ} 00^{\prime} 04^{\prime \prime} \mathrm{W}, 240 \mathrm{~m}$, Subandean Amazon forest, corticolous, 29 July 2008, $A F 11242 \& M K$ (KRAM, LPB).

*Malmidea vinosa (Eschw.) Kalb, Rivas Plata \& Lumbsch

In the Neotropics this lichen is known only from Brazil, Guyana and Florida (U.S.A.) (Cáceres 2007; Lücking 2008; Lücking et al. 2011a).

Specimens examined. BOLIVIA. Dept. La PaZ. Prov. Nor Yungas, entre Cotapata y Yucupi, $16^{\circ} 11^{\prime} 52.5^{\prime \prime} \mathrm{S}$, $67^{\circ} 49^{\prime} 25.0^{\prime \prime} \mathrm{W}, 1475 \mathrm{~m}$, bosque intervenido cercano a la comunidad de Yucupi y a la carretera secundaria de acceso, corticolous, 4 Dec. 2006, $P R \& S$. Gallegos 298 (B, LPB).

\section{*Megalospora admixta (Nyl.) Sipman}

This Neotropical species is known from Brazil, Costa Rica, Colombia, Ecuador, Jamaica and Venezuela (Sipman 1986; Umaña-Tenorio et al. 2002; Nöske \& Sipman 2004).

Specimens eXamined. BOLIVIA. DePt. CochaBAMBA. Prov. Chapare, near Incachaca, $17^{\circ} 12^{\prime} 54^{\prime \prime} \mathrm{S}$, $65^{\circ} 49^{\prime} 30^{\prime \prime} \mathrm{W}, 2028 \mathrm{~m}$, Yungas cloud forest near road, corticolous, 7 July 2009, AF 12982 \& 13006 (KRAM); 
Dept. Santa Cruz. Prov. Manuel María Caballero, El Rincón, $17^{\circ} 49^{\prime} 33^{\prime \prime} \mathrm{S}, 6^{\circ} 38^{\prime} 50^{\prime \prime} \mathrm{W}, 2670 \mathrm{~m}$, Yungas cloud forest, corticolous, 17 Aug. 2012, AF 24435.1 (LPB).

\section{${ }^{*}$ Megaspora verrucosa (Ach.) L. Arcadia}

\& A. Nordin

This widely distributed species (Feuerer 2013) is already known in South America from Argentina, Chile, Colombia, Ecuador and Venezuela (Arvidsson 1991; Marcano et al. 1996; Galloway \& Quilhot 1998; Calvelo \& Liberatore 2002; Sipman et al. 2008).

Specimens eXamined. BOLIVIA. Dept. La Paz. Prov. Franz Tamayo, APOLOBAMBA, near Puyo Puyo, $14^{\circ} 56^{\prime} 55^{\prime \prime} \mathrm{S}, 69^{\circ} 07^{\prime} 58^{\prime \prime} \mathrm{W}, 4795 \mathrm{~m}$, high Andean open vegetation, on terricolous bryophytes, 5 July $2010, A F$ $17687.2 \& P R$ (LPB).

\section{Parmotrema neotropicum Kurok.}

This lichen was recently reported from two localities in Bolivia (Kukwa et al. 2012).

CHEMISTRY. Atranorin, usnic and salazinic acids.

Specimens eXamined. BOLIVIA. DePt. La PAZ. Prov. Larecaja, SW of Itubaya, $15^{\circ} 32^{\prime} 37^{\prime \prime} \mathrm{S}, 68^{\circ} 39^{\prime} 49^{\prime \prime} \mathrm{W}$, $2813 \mathrm{~m}$, Yungas forest, on rocks, 14 May 2011, MK 8543 (LPB, UGDA).

\section{Parmotrema simulans (Hale) Hale}

This species was only recently recorded from one locality in Bolivia (Kukwa et al. 2012).

CHEMISTRY. Atranorin and caperatic acid.

SPECIMENS EXAMINED. BOLIVIA. Dept. TARIJA. Prov. Aniceto Arce, Filo de Sidras, camp of guards, near Tarija, $22^{\circ} 14^{\prime} 50^{\prime \prime} \mathrm{S}, 64^{\circ} 33^{\prime} 28^{\prime \prime} \mathrm{W}, 1064 \mathrm{~m}$, TucumanoBoliviano submontane forest, on bark, 20 Oct. 2010, $A F$ 18435, PR (KRAM, LPB).

\section{*Parmotrema subochraceum Hale}

Previously known only from Brazil, where it is apparently common (Hale 1990a; Benatti \& Marcelli 2009, 2011).

CHEMISTRY. Atranorin and protocetraric acid; medulla partly pigmented orange.
Specimens eXamined. BOLIVIA. Dept. SAnta CRUZ. Prov. José Miguel de Velasco, PN Noel Kempff Mercado, near Florida, $14^{\circ} 35^{\prime} 50^{\prime \prime} \mathrm{S}, 61^{\circ} 50^{\prime} 46^{\prime \prime} \mathrm{W}, 220 \mathrm{~m}$, Cerrado forest, on bark, 15 Dec. 2009, AF 15744 (KRAM, LPB).

Phaeographis dendritica (Ach.) Müll. Arg.

This species has been reported from three Bolivian localities by Kukwa et al. (2013).

CHEMISTRY. Norstictic and connorstictic acids.

Specimens examined. BOLIVIA. Dept. LA PAZ. Prov. Nor Yungas, near Nogalani, $16^{\circ} 12^{\prime} 57^{\prime \prime} \mathrm{S}$, $67^{\circ} 49^{\prime} 15^{\prime \prime} \mathrm{W}, 2168 \mathrm{~m}$, Yungas secondary cloud forest, epiphytic, 2 May 2011, US (LBP, herb. Schiefelbein 3072, 3078); Prov. Nor Yungas, COTAPATA, Chairo, $16^{\circ} 12^{\prime} 07^{\prime \prime} \mathrm{S}, 67^{\circ} 50^{\prime} 05^{\prime \prime} \mathrm{W}, 1300 \mathrm{~m}$, Yungas montano inferior forest, epiphytic, 21 May 2011, US (LPB).

Phaeographis dendroides (Leight.) Müll. Arg.

Recently reported from two localities in Bolivia (Kukwa et al. 2013).

Chemistry. Stictic and hypostictic acids.

Specimens examined. BOLIVIA. Dept. La Paz. Prov. Nor Yungas, COTAPATA, between Tunkini and Chairo, $16^{\circ} 11^{\prime} 00^{\prime \prime} \mathrm{S}, 67^{\circ} 52^{\prime} 00^{\prime \prime} \mathrm{W}$, Yungas montane forest, epiphytic, 22 May 2011, US (LPB).

\section{Phaeographis scalpturata (Ach.) Staiger}

This species has been reported in Bolivia only by Nylander (1859).

Chemistry. No lichen compounds detected by TLC.

Specimens examined. BOLIVIA. DePt. La PaZ. Prov. Nor Yungas, COTAPATA, between Tunkini and Chairo, $16^{\circ} 11^{\prime} 00^{\prime} \mathrm{S}, 67^{\circ} 52^{\prime} 00^{\prime \prime} \mathrm{W}$, Yungas montane forest, epiphytic, 22 May 2011, US (LPB, herb. Schiefelbein 3063).

Phaeophyscia adiastola (Essl.) Essl.

This predominantly Northern Hemisphere lichen is known from East Africa, Europe (France), Canada and the U.S.A. (Esslinger 1977, 2004; Goward et al. 1994; Feuerer 2013). It was recorded 
from Bolivia by Canseco et al. (2006), apparently the first report from the Southern Hemisphere.

Specimens eXamined. BOLIVIA. Dept. Beni. Prov. Cercado, isla de los Caimánes, near San Pedro Nuevo, $14^{\circ} 30^{\prime} 11^{\prime \prime} \mathrm{S}, 64^{\circ} 52^{\prime} 21^{\prime \prime} \mathrm{W}, 155 \mathrm{~m}$, lowland Amazon forest surrounded by los Llanos de Moxos savanna, by lake, on bark, 1 Sept. 2012, MK 12005c (LPB); DEPT. LA PAZ. Prov. Murillo, La Florida, plaza Humboldt, camino a la avenida Costanera, $3251 \mathrm{~m}, 16^{\circ} 32.2^{\prime} \mathrm{S}, 68^{\circ} 05.5^{\prime} \mathrm{W}$, Puna y vegetación Altoandina, on Populus balsamifera, 8 Feb. 2002, A. D. Canseco-Tarifa 129 (B, LPB).

\section{Phaeophyscia endococcinoides (Poelt) Essl.}

This species has been recorded from Bolivia on only three occasions (Feuerer et al. 1998; Flakus et al. 2012a).

Specimens eXamined. BOLIVIA. DePt. LA PAZ. Prov. Bautista Saavedra, $28 \mathrm{~km}$ from Charazani, on the road between Charazani and Apolo, $15^{\circ} 12^{\prime} 43^{\prime \prime} \mathrm{S}$, $68^{\circ} 47^{\prime} 25^{\prime \prime} \mathrm{W}, 1650 \mathrm{~m}$, Yungas montane forest, open area with boulders, on rock, 18 May 2011, MK 8910 (LPB, UGDA).

\section{${ }^{*}$ Phaeophyscia hispidula (Ach.) Essl.}

This species occurs in all continents except Antarctica (Moberg 1993; Esslinger 2004; Feuerer 2013). In South America it is known only from Argentina, Brazil, Ecuador, Peru and Venezuela (Moberg 1993).

Specimens eXAmined. BOLIVIA. DePt. COCHABAMBA. Prov. Ayopaya, $6 \mathrm{~km}$ al sur de Saila Pata, $3050 \mathrm{~m}, 16^{\circ} 55^{\prime} \mathrm{S}, 66^{\circ} 56^{\prime} \mathrm{W}$, cresta en bosque siempreverde, epiphytic, 11 Nov. 1997, KB 878 \& I. Jiménez (B, LPB); DEPT. LA PAZ. Prov. Bautista Saavedra, $10 \mathrm{~km}$ de Charazani hacia Apolo, $2550 \mathrm{~m}, 15^{\circ} 11^{\prime} \mathrm{S}, 68^{\circ} 52^{\prime} \mathrm{W}$, pared rocosa, saxicolous, 31 May 1997, KB et al. 132 (B, LPB); km 202 on the road from Apolo to Charazani, $20 \mathrm{~km}$ from Charazani, $15^{\circ} 12^{\prime} 35^{\prime \prime} \mathrm{S}, 68^{\circ} 51^{\prime} 30^{\prime \prime} \mathrm{W}$, $2260 \mathrm{~m}$, transition Yungas montane forest-cloud forest, on rock, 18 May 2011, MK 8919 (LPB, UGDA).

\section{Phaeophyscia sciastra (Ach.) Moberg}

This species has been reported in Bolivia [as Physcia obscura var. sciastra (Ach.) Nyl.] only by Nylander (1861).

Specimens examined. BOLIVIA. Dept. La PaZ.
Prov. Murillo, La Paz, plaza Los Leones, 3508 m, $16^{\circ} 30.6^{\prime} \mathrm{S}, 68^{\circ} 07.1^{\prime} \mathrm{W}$, espacio amplio con bastante flora ornamental, en Ulmus pumila, 11 July 2008, G.YapuAlcázar 358 (B, LPB).

\section{${ }^{*}$ Phlyctella andensis (Nyl.) Nyl.}

Phlyctis andensis Nyl.

This species was reported in the Neotropics from Brazil, Colombia, Costa Rica, Ecuador and Uruguay (Osorio 1972; Etayo 2002; Umaña-Tenorio et al. 2002; Nöske \& Sipman 2004; Sipman et al. 2008; Feuerer 2013).

SPECIMENS EXAmined. BOLIVIA. DePt. LA PAZ. Prov. Nor Yungas, Cotapata, 1 hora caminando desde Sandillani hacia Bajo Hornuni. $1520 \mathrm{~m}, 16^{\circ} 11^{\prime} 06^{\prime \prime} \mathrm{S}$, $67^{\circ} 49^{\prime} 30^{\prime \prime} \mathrm{W}$, bosque muy húmedo, corticolous, 13 Oct. 2006, PR et al. 163 (B, LPB, with a specimen of Megalospora tuberculosa).

\section{*Phyllopsora corallina (Eschw.) Müll. Arg. s.str.}

This is a widespread tropical lichen (Brako 1991). In South America it has been recorded from Argentina, Brazil, Ecuador, French Guiana, Guyana, Peru, Surinam and Venezuela (Brako 1991; Calvelo \& Liberatore 2002).

CHEMISTRY. No lichen substances detected by TLC.

SPECIMENS EXAMINEd. BOLIVIA. DePt. Beni. Prov. Iténez, Maconere by río San Martín, $13^{\circ} 16^{\prime} 45^{\prime \prime} \mathrm{S}$, $63^{\circ} 21^{\prime} 49^{\prime \prime} \mathrm{W}, 145 \mathrm{~m}$, lowland Amazon forest, corticolous, 20 Aug. 2008, $A F 12792 \&$ PR (KRAM, LPB); DEPT. TARIJA. Prov. Aniceto Arce, Papachacra, $21^{\circ} 41^{\prime} 54^{\prime \prime} \mathrm{S}$, $64^{\circ} 29^{\prime} 28^{\prime \prime} \mathrm{W}, 2056 \mathrm{~m}$, Tucumano-Boliviano montane forest, corticolous, 27 Nov. 2010, $A F 20015$ \& JQ (LPB).

\section{* Phyllopsora furfuracea Zahlbr.}

This is a widely distributed species in the tropics and subtropics (Brako 1991; Timdal 2008b); in South America it has been reported from Brazil, Colombia, Ecuador, French Guiana, Guyana, Peru, and Venezuela (Brako 1991; Timdal 2008b; Sipman et al. 2008).

CHEMISTRY. No lichen substances detected by TLC. 
Specimens examined. BOLIVIA. Dept. La PaZ. Prov. Nor Yungas, COTAPATA, near Urpuma colony, $16^{\circ} 13^{\prime} 20^{\prime \prime} \mathrm{S}, 67^{\circ} 52^{\prime} 34^{\prime \prime} \mathrm{W}, 1989 \mathrm{~m}$, Yungas montane forest, corticolous, 30 June 2010, $A F$ 17214, $17317 \& P R$ (KRAM, LPB).

\section{${ }^{*}$ Phyllopsora isidiotyla (Vain.) Riddle}

Reported here for the first time from Bolivia, this lichen is already known from Brazil, Costa Rica, the southern U.S.A., Venezuela and northern Australia (Brako 1991; Umaña-Tenorio et al. 2002; Elix 2009b).

CHEMISTRY. No lichen substances detected by TLC.

SPECIMENS EXAMINED. BOLIVIA. DEPT. TA-

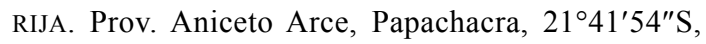
$64^{\circ} 29^{\prime} 28^{\prime \prime} \mathrm{W}, 2056 \mathrm{~m}$, Tucumano-Boliviano montane forest, corticolous, 27 Nov. 2010, AF 19958 \& JQ (KRAM, LPB).

\section{*Phyllopsora kalbii Brako}

This species has been reported from Brazil, the Dominan Republic, the southern U.S.A., Kenya and Tanzania (Brako 1991).

CHEMISTRY. No lichen substances detected by TLC.

SPECIMENS EXAmined. BOLIVIA. DePt. SANTA CRUZ. Prov. Cordillera, KAA-IYA, by Tucavaca colony, $18^{\circ} 31^{\prime} 40^{\prime \prime} \mathrm{S}, 60^{\circ} 41^{\prime} 26^{\prime \prime} \mathrm{W}, 314 \mathrm{~m}$, Chiquitano forest, corticolous, 4 Dec. 2010, $A F 19221 \& J Q$ (KRAM, LPB).

\section{*'Phyllopsora parvifolia (Pers.) Müll. Arg.}

So far reported from Africa, Australia, the Neotropics, and the southern U.S.A. (Brako 1991). In South America it was collected in Argentina, Brazil, Colombia, Ecuador, Paraguay, Uruguay and Venezuela (Brako 1991; Sipman et al. 2008).

CHEMISTRY. No lichen substances detected by TLC.

SPECIMENS EXAMINED. BOLIVIA. DEPT. COCHABAMBA. Prov. Carrasco, CARRASCO, near Sehuencas,

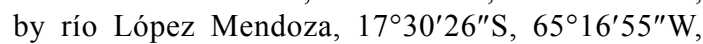
$2226 \mathrm{~m}$, Yungas cloud forest, corticolous, 22 July 2008, $A F$ 10697, MK \& PR (KRAM, LPB); DEPT. LA PAZ. Prov. Nor Yungas, Cotapata cerca a la comunidad del
Chairo, $1370 \mathrm{~m}, 16^{\circ} 13^{\prime} 18^{\prime \prime} \mathrm{S}, 67^{\circ} 52^{\prime} 36^{\prime \prime} \mathrm{W}$, manchón de bosque cercano al río Huarinilla, corticolous, 11 May 2006, PR et al. 84 (B, LPB); DEPT. TARIJA. Prov. Aniceto Arce, Filo de Sidras, $22^{\circ} 14^{\prime} 50^{\prime \prime} \mathrm{S}, 64^{\circ} 33^{\prime} 28^{\prime \prime} \mathrm{W}$, $1064 \mathrm{~m}$, Tucumano-Boliviano submontane forest, corticolous, 22 Nov. 2010, AF 18496 (KRAM, LPB).

\section{*Physcia atrostriata Moberg}

This is a widely distributed tropical, subtropical and Mediterranean lichen (Feuerer 2013). In South America it has been reported from Argentina, Brazil, Colombia, Ecuador, French Guiana, Guyana, Peru, Surinam and Venezuela (Moberg 1990).

Specimens eXamined. BOLIVIA. DePt. La PaZ. Prov. Sud Yungas, Alto Beni, Mapurichuqui, towards Villazón, $450 \mathrm{~m}, 15^{\circ} 31.749^{\prime} \mathrm{S}, 67^{\circ} 23.058^{\prime} \mathrm{W}$, plantation, on Theobroma cacao, 22 April 1999, N. Derakshani 41 (B, GOET).

\section{*Physcia pachyphylla Müll. Arg.}

Previously known only from Argentina, Brazil, Costa Rica, Ecuador, Guatemala, Peru, Uruguay and Venezuela (Moberg 1990). The Bolivian localities represent the altitudinal maximum for the species.

Specimens eXamined. BOLIVIA. DePt. La PaZ. Prov. Murillo. La Paz, plaza Montenegro, Achumani, $3340 \mathrm{~m}, 16^{\circ} 31.9^{\prime} \mathrm{S}, 68^{\circ} 04.6^{\prime} \mathrm{W}$, área verde recreacional, on Pinus radiata, 1 July 2008, G. Yapu-Alcázar 178 (B, LPB); plaza del Minero, $3609 \mathrm{~m}, 16^{\circ} 30.3^{\prime} \mathrm{S}, 68^{\circ} 06.6^{\prime} \mathrm{W}$, área verde, on Populus balsamifera, 7 July 2008, G. Yapu-Alcázar 277 (B, LPB); plaza Los Leones, $3508 \mathrm{~m}$, $16^{\circ} 30.5^{\prime} \mathrm{S}, 68^{\circ} 06.9^{\prime} \mathrm{W}$, área verde deficiente, on Ulmus pumila, 8 July 2008, G. Yapu-Alcázar 315 (B, LPB).

\section{*Physcia poncinsii Hue}

This species has been reported from Central and South America (Argentina, Brazil, Chile, Cuba, Ecuador, Colombia, Peru, Uruguay and Venezuela), Australia, East Africa, New Zealand and Papua New Guinea (Moberg 1990, Aptroot \& Sipman 1991; Osorio 1992; Calvelo \& Liberatore 2002; Galloway 2007; Elix 2009c; Feuerer 2013).

Specimens examined. BOLIVIA. DePt. La PaZ. Prov. Sud Yungas, Alto Beni, Belén, $680 \mathrm{~m}, 15^{\circ} 33.80^{\prime} \mathrm{S}$, $67^{\circ} 26.68^{\prime} \mathrm{W}$, plantation, on Citrus bark, 15 June 1999, N. Derakshani 8 (B, GOET). 


\section{${ }^{*}$ Physcia sorediosa (Vain.) Lynge}

This species was mostly reported from South America (Argentina, Brazil, Colombia, Ecuador, French Guiana, Guyana, Paraguay, Surinam, Uruguay and Venezuela). It is also known from Africa, Asia, Australia, North America, Papua New Guinea and the Azores (Moberg 1990, Aptroot \& Sipman 1991; Elix 2009c; Feuerer 2013).

Specimens examined. BOLIVIA. Dept. La PaZ. Prov. Sud Yungas, Alto Beni, Mapurichuqui, towards Villazón, $450 \mathrm{~m}, 15^{\circ} 31.749^{\prime} \mathrm{S}, 67^{\circ} 23.058^{\prime} \mathrm{W}$, plantation, on Theobroma cacao, 22 April 1999, N. Derakshani 57 (B, GOET).

\section{${ }^{*}$ Physcidia matogrossensis Kalb \& Elix}

This species was previously known only from Brazil (Kalb \& Elix 1995).

SPECimens eXamined. BOLIVIA. DePt. SAnta Cruz. Prov. José Miguel de Velasco, sendero de goma, near Florida, $14^{\circ} 37^{\prime} 48^{\prime \prime} \mathrm{S}, 61^{\circ} 12^{\prime} 02^{\prime \prime} \mathrm{W}, 170 \mathrm{~m}$, lowland Amazon secondary forest, corticolous, 16 Dec. 2009, $A F 16098 \&$ \& (KRAM, LPB).

\section{*Placidium lacinulatum (Ach.) Breuss}

This rather widely distributed species is already known in South America from Argentina, Brazil and Peru (Breuss 1993; Prieto et al. 2010).

SPECIMENS EXAMINED. BOLIVIA. DEPT. COCHABAMBA. Prov. Ayopaya, $20 \mathrm{~km}$ de Cocapata hacia Cotacajes, $2000 \mathrm{~m}, 16^{\circ} 46^{\prime} \mathrm{S}, 66^{\circ} 44^{\prime} \mathrm{W}$, bosque semideciduo intervenido, epiphytic, 15 May 1997, KB et al. $114 a$ (B, LPB).

\section{Platygramme caesiopruinosa (Fée) Fée}

This species was only recently reported from Bolivia (Kukwa et al. 2013).

CHEMISTRY. No lichen compounds detected by TLC.

Specimens examined. BOLIVIA. Dept. LA PAz. Prov. Nor Yungas, between Coroico and La Paz, $16^{\circ} 13^{\prime} 33^{\prime \prime} \mathrm{S}, 67^{\circ} 49^{\prime} 20^{\prime \prime} \mathrm{W}, 2600 \mathrm{~m}$, Yungas secondary cloud forest, on tree, 23 May 2011, US (LPB); COTAPATA, Santa Catalina, $16^{\circ} 11^{\prime} 12^{\prime \prime} \mathrm{S}, 67^{\circ} 52^{\prime} 07^{\prime \prime} \mathrm{W}$, Yungas montane forest, on tree, 22 May 2011, US (LPB, herb. Schiefelbein 3046, 3048).
*Polymeridium albidum (Müll. Arg.) R. C. Harris

This rare tropical lichen was previously known in South America only from Brazil (Harris 1986). It was also reported from Costa Rica, Papua New Guinea, Australia and Thailand (Aptroot et al. 1997; Wolseley et al. 2002; Breuss \& Neuwirth 2007).

SPecimens eXamined. BOLIVIA. DePt. La PAZ. Prov. Sud Yungas, Alto Beni Sapecho, Cooperativa Sapecho, $500 \mathrm{~m}, 15^{\circ} 32^{\prime} \mathrm{S}, 67^{\circ} 20^{\prime} \mathrm{W}$, barbecho de 12 años, epiphytic, 2 Aug. 1999, A. Acebey \& T. Krömer $995 b$ (B, LPB).

*Polymeridium subcinereum (Nyl.) R. C. Harris

This species was reported in South America from Brazil and Guyana (Feuerer 2013). It is also known from France, the U.S.A. and Australia (Aptroot 1998; Tucker \& Harris 1980; McCarthy 2013).

Specimens eXamined. BOLIVIA. DePt. LA PAZ. Prov. Bautista Saavedra, cerro Asunta Pata, $1500 \mathrm{~m}$, $15^{\circ} 05^{\prime} \mathrm{S}, 68^{\circ} 29^{\prime} \mathrm{W}$, bosque siempreverde, epiphytic, 21 June 1997, KB et al. 200 (B, LPB).

${ }^{*}$ Porina tetracerae (Afzel.) Müll. Arg.

The lichen is widely distributed, mainly in the tropics (see the distribution map in McCarthy 2003). In South America it is known from Argentina, Brazil, Colombia, Ecuador, French Guiana, Guyana, Peru, Surinam and Venezuela (Aptroot \& Sipman 1993; Calvelo \& Liberatore 2002; Cáceres 2007; Neuwirth 2007; Lücking 2008; Sipman et al. 2008).

Specimens eXAmined. BOLIVIA. Dept. LA PAZ. Prov. Nor Yungas, Cotapata cerca a la comunidad del Chairo, $1370 \mathrm{~m}, 16^{\circ} 13^{\prime} 18^{\prime \prime} \mathrm{S}, 67^{\circ} 52^{\prime} 36^{\prime \prime} \mathrm{W}$, manchón de bosque cercano al río Huarinilla, epiphytic, 11 May 2006, PR et al. 193 (B, LPB).

\section{* Pseudopyrenula subnudata Müll. Arg.}

This pantropical lichen was known previously in South America only from Brazil (Müller 1883; Aptroot 2002).

Specimens examined. BOLIVIA. Dept. Santa Cruz. Prov. Cordillera, $350 \mathrm{~km}$ on the road from 
Tucavaca to Roboré, $18^{\circ} 37^{\prime} 56^{\prime \prime} \mathrm{S}, 59^{\circ} 36^{\prime} 50^{\prime \prime} \mathrm{W}, 370 \mathrm{~m}$, transition Chiquitano-Chaqueño forest, on twig, 4 Dec. 2011, MK 10552a \& $10531 b$ (LPB, UGDA).

\section{${ }^{* *}$ Psiloparmelia arhizinosa Hale}

Previously known only from southern Africa (Lesotho) (Elix \& Nash 1992).

ChEMISTRy. Atranorin, usnic and constipatic acids.

SPeCimens eXamined. BOLIVIA. DePt. LA PAZ. Prov. Bautista Saavedra, APOLOBAMBA, near Taypi Cañuma, $15^{\circ} 03^{\prime} 20^{\prime \prime} \mathrm{S}, 69^{\circ} 09^{\prime} 07^{\prime \prime} \mathrm{W}, 4506 \mathrm{~m}$, high Andean open vegetation, saxicolous, 5 July $2010, A F 17526 \& P R$ (KRAM, LPB); DEPT. ORURO. Prov. Sajama, SAJAMA, near Sajama, $18^{\circ} 07^{\prime} 49^{\prime \prime} \mathrm{S}, 68^{\circ} 56^{\prime} 54^{\prime \prime} \mathrm{W}, 4437 \mathrm{~m}$, Puna Sureña, shrubland dominated by Baccharis, saxicolous, 18 June 2010, $A F 16461 \& P R$ (LPB).

\section{${ }^{*}$ Psiloparmelia dichotoma Elix \& T. H. Nash}

Previously known only from the type locality in Peru (Elix \& Nash 1992).

\section{CHEMISTRY: Atranorin and usnic acid.}

SPECIMENS EXAMINED. BOLIVIA. DEPT. ORURO. Prov. Sajama, SAJAMA, Huincurata, near Sajama, $18^{\circ} 07^{\prime} 01^{\prime \prime} \mathrm{S}, 68^{\circ} 58^{\prime} 00^{\prime \prime} \mathrm{W}, 4301 \mathrm{~m}$, Puna Sureña, Polylepis forest (Queñuales), saxicolous, 19 June 2010, $A F 16546 \& P R$ (KRAM, LPB).

\section{* Psiloparmelia diffractaica Elix \& T. H. Nash}

Known so far only from type locality in Argentina (Elix \& Nash 1992).

CHEMISTRY: Diffractaic and usnic acids.

SPECIMENS EXAMINED. BOLIVIA. DEPT. ORURO. Prov. Sajama, SAJAMA, Aguas Calientes, near Sajama, $18^{\circ} 05^{\prime} 29^{\prime \prime} \mathrm{S}, 69^{\circ} 02^{\prime} 28^{\prime \prime} \mathrm{W}, 4448 \mathrm{~m}$, Puna Sureña, shrubland dominated by Baccharis, saxicolous, 18 June 2010, AF 16521 \& PR (KRAM, LPB); DEPT. Potosí. Prov. Nor Lípez, Pinturas Rupestres, near Mallku Villamar, $21^{\circ} 46^{\prime} 20^{\prime \prime} \mathrm{S}, 67^{\circ} 29^{\prime} 05^{\prime \prime} \mathrm{W}, 4038 \mathrm{~m}$, open semidesert high Andean area, on volcanic rock, 6 Dec. 2009, $A F 14759 \& P R$ (LPB, herb. Flakus).

Psiloparmelia flavobrunnea (Müll. Arg.) Elix \& T. H. Nash

This species was previously recorded from two localities in Bolivia (Elix \& Nash 1992).
CHEMISTRY. Atranorin, usnic, protoconstipatic, constipatic $( \pm)$, dehydroxyconstipatic $( \pm)$ and unknown fatty acid $( \pm)$.

Specimens examined. BOLIVIA. Dept. LA PAZ. Prov. Franz Tamayo, APOLOBAMBA, near Puyo Puyo, $14^{\circ} 56^{\prime} 55^{\prime \prime} \mathrm{S}, 6^{\circ} 07^{\prime} 58^{\prime \prime} \mathrm{W}, 4888 \mathrm{~m}$, high Andean open vegetation, on schist rock, 5 July 2010, AF 17566, 17581 \& PR (KRAM, LPB); Prov. Manco Kapac, near Copacabana Mt Horca del Inca, $16^{\circ} 10^{\prime} 15^{\prime \prime} \mathrm{S}, 69^{\circ} 05^{\prime} 05^{\prime \prime} \mathrm{W}$, 3974 m, high Andean Puna, saxicolous, 18 June 2006, AF 8608 \& 8666 (LPB); Prov. Murillo, near Cumbre pass, $16^{\circ} 20^{\prime} 14^{\prime \prime} \mathrm{S}, 68^{\circ} 02^{\prime} 20^{\prime \prime} \mathrm{W}, 4672 \mathrm{~m}$, high Andean Puna, on schist rock, 13 May 2006, AF 5714.1 (LPB); Prov. Omasuyos, Tajani cerca Achacachi, $15^{\circ} 36^{\prime} 49^{\prime \prime} \mathrm{S}$, $69^{\circ} 04^{\prime} 02^{\prime \prime} \mathrm{W}, 3869 \mathrm{~m}$, Puna Húmeda, on quartzite rock, 6 July 2010, AF 17796, 17806 \& PR (KRAM, LPB); DEPT. ORURO. Prov. Sajama, SAJAMA, Ladera de la Quebrada Kohiri, 4309 m, 1807'07"S, 68 $56^{\prime} 06^{\prime \prime} \mathrm{W}$, bosque de Polylepis tarapacana en ladera con piedras grandes, sobre roca, 21 Nov. 2007, RIM et al. 4387 (B, LPB); ibidem, $4406 \mathrm{~m}, 18^{\circ} 07^{\prime} 08^{\prime \prime} \mathrm{S}, 68^{\circ} 56^{\prime} 05^{\prime \prime} \mathrm{W}$, sobre roca. 21 Nov. 2007, RIM et al. 4393 (B, LPB);

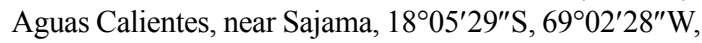
$4448 \mathrm{~m}$, Puna Sureña, shrubland dominated by Baccharis, saxicolous, 18 June 2010, $A F \& P R$ 16525, 16527, 16528 (KRAM, LPB); Huincurata, near Sajama, $18^{\circ} 07^{\prime} 01^{\prime \prime} \mathrm{S}, 68^{\circ} 58^{\prime} 00^{\prime \prime} \mathrm{W}, 4301 \mathrm{~m}$, Puna Sureña, $P o-$ lylepis forest (Queñuales), saxicolous, 19 June 2010, $A F 16545,16550,16555,16597 \&$ \&R (KRAM, LPB); Jecha K'ala, $18^{\circ} 09^{\prime} 52^{\prime \prime} \mathrm{S}, 68^{\circ} 49^{\prime} 08^{\prime \prime} \mathrm{W}, 4184 \mathrm{~m}$, Puna Sureña, grassland vegetation, saxicolous, 20 June 2010, AF 16607, 16610, 1664,7 16666 \& PR (KRAM, LPB); near Sajama, $18^{\circ} 07^{\prime} 49^{\prime \prime} \mathrm{S}, 68^{\circ} 56^{\prime} 54^{\prime \prime} \mathrm{W}, 4437 \mathrm{~m}$, Puna Sureña, shrubland dominated by Baccharis, saxicolous, 18 June 2010, AF 16460, 16462, 16508 \& PR (KRAM); DEPT. PoTOSí. Prov. Nor Lípez, Pinturas Rupestres, near Mallku Villamar, $21^{\circ} 46^{\prime} 20^{\prime \prime} \mathrm{S}, 67^{\circ} 29^{\prime} 05^{\prime \prime} \mathrm{W}, 4038 \mathrm{~m}$, open semi-desert high Andean area, saxicolous, 6 Dec. 2009, $A F 14775$ \& $P R$ (KRAM, LPB).

\section{${ }^{*}$ Psiloparmelia norstictica Elix \& T. H. Nash}

This species was reported previously from Argentina, Ecuador and Peru (Elix \& Nash 1992).

Chemistry. Atranorin (trace), usnic and norstictic acids.

Specimens eXAmined. BOLIVIA. DePt. OruRo. Prov. Sajama, SAJAMA, Huincurata, near Sajama, $18^{\circ} 07^{\prime} 01^{\prime \prime} \mathrm{S}, 68^{\circ} 58^{\prime} 00^{\prime \prime} \mathrm{W}, 4301 \mathrm{~m}$, Puna Sureña, Po- 
lylepis forest (Queñuales), saxicolous, 19 June 2010, $A F 16544 \&$ \&R (KRAM, LPB, herb. Flakus).

\section{*Psiloparmelia sorediosa Elix \& T. H. Nash}

Previously this species was known only from the type locality in Argentina (Elix \& Nash 1992).

Chemistry. Atranorin (trace, \pm ), usnic and norstictic acids.

SPeCimens eXAmined. BOLIVIA. DePt. ORURo. Prov. Sajama, SAJAMA, Huincurata, near Sajama, $18^{\circ} 07^{\prime} 01^{\prime \prime} \mathrm{S}, 68^{\circ} 58^{\prime} 00^{\prime \prime} \mathrm{W}, 4301 \mathrm{~m}$, Puna Sureña, Polylepis forest (Queñuales), saxicolous, 19 June 2010, $A F 16546.1,16582 \&$ \& (KRAM, LPB); Jecha K'ala, $18^{\circ} 09^{\prime} 52^{\prime \prime} \mathrm{S}, 68^{\circ} 49^{\prime} 08^{\prime \prime} \mathrm{W}, 4184 \mathrm{~m}$, Puna Sureña, grassland vegetation, saxicolous, 20 June 2010, $A F$ 16605, $16656,16695,16706.2 \&$ PR (KRAM, LPB); near Sajama, $18^{\circ} 07^{\prime} 49^{\prime \prime} \mathrm{S}, 68^{\circ} 56^{\prime} 54^{\prime \prime} \mathrm{W}, 4437 \mathrm{~m}$, Puna Sureña, shrubland dominated by Baccharis, saxicolous, 18 June 2010, $A F 16474 \& P R$ (LPB, herb. Flakus).

\section{${ }^{*}$ Psiloparmelia subcrustosa Elix \& T. H. Nash}

This lichen was previously known only from the type locality in Peru (Elix \& Nash 1992).

CHEMISTRY. Atranorin and isousnic acid.

Specimens examined. BOLIVIA. Dept. La Paz. Prov. Franz Tamayo, APOLOBAMBA, near Puyo Puyo, $14^{\circ} 56^{\prime} 55^{\prime \prime} \mathrm{S}, 6^{\circ} 07^{\circ} 58^{\prime \prime} \mathrm{W}, 4888 \mathrm{~m}$, high Andean open vegetation, on schist rock, 5 July 2010, $A F$ \& PR 1757617623 (KRAM, LPB); DEPT. ORURO. Prov. Sajama, SAJAMA, Aguas Calientes, near Sajama, $18^{\circ} 05^{\prime} 29^{\prime \prime} \mathrm{S}, 6^{\circ} 02^{\prime} 28^{\prime \prime} \mathrm{W}, 4448 \mathrm{~m}$, Puna Sureña, shrubland dominated by Baccharis, on rock, 18 June 2010, $A F$ 16517, 16532, 16520, 16524 \& PR (KRAM, LPB, UGDA); Huincurata, near Sajama, $18^{\circ} 07^{\prime} 01^{\prime \prime} \mathrm{S}$, $68^{\circ} 58^{\prime} 00^{\prime \prime} \mathrm{W}, 4301 \mathrm{~m}$, Puna Sureña, Polylepis forest (Queñuales), saxicolous, 19 June 2010, $A F$ 16570, $16585,16587,16600 \& P R$ (KRAM, LPB); Jecha $\mathrm{K}^{\prime}$ ala, $18^{\circ} 09^{\prime} 52^{\prime \prime} \mathrm{S}, 68^{\circ} 49^{\prime} 08^{\prime \prime} \mathrm{W}, 4184 \mathrm{~m}$, Puna Sureña, grassland vegetation, saxicolous, 20 June 2010, $A F 16619,16633 \& P R$ (KRAM, LPB); near Sajama, $18^{\circ} 07^{\prime} 49^{\prime \prime} \mathrm{S}, 68^{\circ} 56^{\prime} 54^{\prime \prime} \mathrm{W}, 4437 \mathrm{~m}$, Puna Sureña, shrubland dominated by Baccharis, saxicolous, 18 June 2010, AF 16459, 16500, 16501, 16512 \& PR (KRAM); DePt. TARIJA. Prov. Méndez, Cuesta de Sama, near Las Antenas, $21^{\circ} 29^{\prime} 59^{\prime \prime} \mathrm{S}, 64^{\circ} 54^{\prime} 46^{\prime \prime} \mathrm{W}, 3850 \mathrm{~m}$, high Andean vegetation, saxicolous, 6 Aug. 2012, AF 23885 (KRAM, LPB).

\section{*Pyrenula aggregata (Fée) Fée}

This widespread Pantropical species has been reported in the Neotropics from Costa Rica and Paraguay (Müller 1888; Aptroot et al. 2008; Aptroot 2012).

Specimens examined. BOLIVIA. Dept. La PaZ. Prov. Sud Yungas, Alto Beni, Nueva Israel, $540 \mathrm{~m}$, $15^{\circ} 34.77^{\prime} \mathrm{S}, 67^{\circ} 15.91^{\prime} \mathrm{W}$, plantation, on Theobroma cacao, 25 June 1999, N. Derakshani 32 (B, GOET).

\section{*Pyrenula anomala (Ach.) Vain.}

This pantropical species has been reported in South America from Brazil, Colombia, Ecuador (the Galapagos Islands), French Guinana, Guyana and Venezuela (Sipman 1992a; Cáceres 2007; Sipman et al. 2008; Bungartz et al. 2013; Aptroot 2012; Feuerer 2013).

CHEMISTRY. Not tested by TLC; thallus UV-.

Specimens examined. BOliVia. Dept. Beni. Prov. Marbán, near road from Camiaco to San Lorenzo, $15^{\circ} 14^{\prime} 49^{\prime \prime} \mathrm{S}, 64^{\circ} 49^{\prime} 11^{\prime \prime} \mathrm{W}, 160 \mathrm{~m}$, lowland Amazon forest surrounded by los Llanos de Moxos savanna, on bark, 30 Aug. 2012, MK $11956 b$ (LPB, UGDA).

\section{*** Pyrenula laetior Müll. Arg.}

Previously this Neotropical taxon was known only from Costa Rica and Cuba (Müller 1885; Aptroot et al. 2008; Aptroot 2012). This new Bolivian record is also the first from the Southern Hemisphere.

Specimens eXamined. BOLIVIA. Dept. La PaZ. Prov. Sud Yungas, Alto Beni, road crossing TarapacaTupiza, $440 \mathrm{~m}, 15^{\circ} 33.81^{\prime} \mathrm{S}, 67^{\circ} 20.79^{\prime} \mathrm{W}$, plantation, on Theobroma cacao, 11 June 1999, N. Derakshani 31 (B, GOET).

\section{*Pyrenula mamillana (Ach.) Trevis.}

This pantropical species has been reported in South America from Brazil, Colombia and Guyanas (Zahlbruckner 1922; Hekking \& Sipman 1988; Aptroot et al. 2008; Sipman et al. 2008; Aptroot 2012).

Specimens eXamined. BOliVia. Dept. Beni. Prov. Yacuma, La Trapiche, E.B. BENI, E of San Borja, 
100 m, on bark, 20 Oct. 1988, S. Stab LB-207 (B); DePT. La Paz. Prov. Sud Yungas, Alto Beni, Sapecho, $405 \mathrm{~m}, 15^{\circ} 30.79^{\prime} \mathrm{S}, 67^{\circ} 30.79^{\prime} \mathrm{W}$, plantation, on Theobroma cacao, 22 June 1999, N. Derakshani 30 (B, GOET).

\section{*Pyrenula mastophoroides (Nyl.) Zahlbr.}

This Neotropical species was reported previously from Colombia and Costa Rica (Aptroot et al. 2008; Sipman et al. 2008).

Specimens examined. BOLIVIA. Dept. La PaZ. Prov. Nor Yungas, Cotapata, a la comunidad de Yucupi y a la carretera secundaria de acceso, $16^{\circ} 11^{\prime} 52^{\prime \prime} \mathrm{S}$, $67^{\circ} 49^{\prime} 25^{\prime \prime} \mathrm{W}, 1475 \mathrm{~m}$, bosque intervenido cercano, corticolous, 4 Dec. 2006, PR \& S. Gallegos 307 (B, LPB).

\section{*Pyrenula septicollaris (Eschw.) R. C. Harris}

This pantropical species has been reported in South America from Brazil, Colombia, French Guiana and Paraguay (Malme 1924; Hekking \& Sipman 1988; Sipman et al. 2008; Aptroot 2012).

SPECIMENS EXAMINED. BOLIVIA. DePt. LA PAZ. Prov. Sud Yungas, Alto Beni, Nueva Israel, $540 \mathrm{~m}$, $15^{\circ} 34.77^{\prime} \mathrm{S}, 67^{\circ} 15.91^{\prime} \mathrm{W}$, plantation, on Theobroma cacao, 25 June 1999, N. Derakshani 43 (B, GOET).

\section{*Pyxine physciiformis (Malme) Imshaug}

P. caesiopruinosa (Tuck.) Imshaug

Pyxine caesiopruinosa and P. physciiformis were treated as distinct species by Kalb (1987) but in more recent times they are considered conspecific (e.g., Elix 2009c). Aptroot (1987) regarded $P$. caesiopruinosa as a synonym of $P$. albovirens (G. Meyer) Aptroot, but Amtoft (2002) proved that they are different species.

This lichen was reported in South America from Brazil, Colombia and Venezuela, and further north from Costa Rica, the Dominican Republic, Mexico and the southeastern U.S.A. (Culberson \& Hale 1965; Kalb 1987; Sipman et al. 2008).

CHEMISTRY. Not tested by TLC; cortex UV+ orange-yellow; medulla with pale salmon-pink pigment reacting $\mathrm{K}+$ violet.
Specimens eXamined. BOLIVIA. DePt. LA PAZ. Prov. Franz Tamayo, near Yuyo, $15^{\circ} 07^{\prime} 05^{\prime \prime} \mathrm{S}$, $68^{\circ} 20^{\prime} 22^{\prime \prime} \mathrm{W}, 1150 \mathrm{~m}$, Preandean Amazon forest, on bark, 16 May 2011, MK 8700 (LPB, UGDA).

\section{${ }^{*}$ Ramalina calcarata Krog \& Swinscow}

Known only from Brazil in South America (Kashiwadani \& Kalb 1993); this species is also known from East Africa (Krog \& Swinscow 1974).

SPeCimens eXamined. BOliVia. DePt. CoCHABAMBA. Prov. Ayopaya, $20 \mathrm{~km}$ de Cocapata hacia Cotacajes, 2000-2100 m, 16 $6^{\circ} 46^{\prime} \mathrm{S}, 66^{\circ} 44^{\prime} \mathrm{W}$, bosque semideciduo intervenido, epiphytic, 15 \& 17 May 1997 , $K B$ et al. $76,77 \& 121$ (B, LPB).

\section{${ }^{*}$ Ramalina cochlearis Zahlbr.}

This Neotropical lichen has been reported from Brazil, Peru and Venezuela (Kashiwadani \& Kalb 1993; Marcano \& Morales Méndez 1994).

SPECIMENS EXAMINED. BOLIVIA. DEPT. COCHABAMBA. Prov. Ayopaya, $10 \mathrm{~km}$ de Cocapata hacia Cotacajes, $2750 \mathrm{~m}, 16^{\circ} 38^{\prime} \mathrm{S}, 66^{\circ} 41^{\prime} \mathrm{W}$, bosque húmedo, epiphytic, 11 May 1997, KB et al. 34 (B, LPB).

\section{Ramalina peruviana Ach.}

This species has been reported in Bolivia only by Feuerer (2010).

Specimens examined. BOLIVIA. DePt. CochaBAMBA. Prov. Ayopaya, $10 \mathrm{~km}$ de Cocapata hacia Cotacajes, 2600-2700 m, $16^{\circ} 38^{\prime} \mathrm{S}, 66^{\circ} 41^{\prime} \mathrm{W}$, bosque húmedo, on decaying wood, 11 \& 14 May 1997, KB et al. 23 \& 69 (LPB); $20 \mathrm{~km}$ de Cocapata hacia Cotacajes, $2000 \mathrm{~m}$, $16^{\circ} 46^{\prime} \mathrm{S}, 66^{\circ} 44^{\prime} \mathrm{W}$, bosque semideciduo intervenido, epiphytic, 15 May 1997, KB et al. 111 (B, LPB); DEPT. LA PAZ. Prov. Bautista Saavedra, $15 \mathrm{~km}$ de Camata hacia Apolo, $1300 \mathrm{~m}, 15^{\circ} 13^{\prime} \mathrm{S}, 68^{\circ} 41^{\prime} \mathrm{W}$, bosque semideciduo, epiphytic, 24 June 1997, KB et al. 221 (LPB); $15 \mathrm{~km}$ de Charazani hacia Apolo, $2400 \mathrm{~m}, 15^{\circ} 11^{\prime} \mathrm{S}$, $68^{\circ} 52^{\prime} \mathrm{W}$, bosque húmedo secundario, epiphytic, 3 July 1997, KB et al. $332 b$ (LPB).

\section{${ }^{*}$ Ramalina reducta Krog \& Swinscow}

This species has been reported from Africa and South America (Colombia and Venezuela) 
(Marcano \& Morales Méndez 1993; Sipman et al. 2008).

Specimens eXamined. BOLIVIA. DePt. La PaZ. Prov. Bautista Saavedra, near Charazani, $15^{\circ} 10^{\prime} 39^{\prime \prime}$ S, $68^{\circ} 56^{\prime} 36^{\prime \prime} \mathrm{W}, 2730 \mathrm{~m}$, Yungas montane forest, on twigs, 18 May 2011, MK 8822 (LPB, UGDA); Prov. Murillo, La Paz, Cota-Cota, Campus Universitario, $16^{\circ} 32.2^{\prime} \mathrm{S}$, $68^{\circ} 04.0^{\prime} \mathrm{W}, 3582 \mathrm{~m}$, Puna y vegetación Altoandina, on Schinus molle, 8 Feb. 2002, A. D. Canseco-Tarifa 141 (B, LPB).

\section{${ }^{*}$ Sipmaniella sulphureofusca (Fée) Kalb}

This species is known from the Neotropics (Costa Rica, Colombia and Jamaica) and Réunion (Sipman 1983; Umaña-Tenorio et al. 2002; Sipman et al. 2008; Kalb et al. 2009).

Specimens examined. BOLIVIA. DePt. LA PaZ. Prov. Nor Yungas, Cotapata, $16^{\circ} 11^{\prime} 06^{\prime \prime} \mathrm{S}, 67^{\circ} 49^{\prime} 30^{\prime \prime} \mathrm{W}$, $1520 \mathrm{~m}$, bosque muy húmedo, corticolous, 13 Oct. 2006, PR et al. 159 (B, LPB); Prov. Nor Yungas, COTAPATA, near Urpuma colony, $16^{\circ} 13^{\prime} 20^{\prime \prime} \mathrm{S}, 67^{\circ} 52^{\prime} 34^{\prime \prime} \mathrm{W}, 1989 \mathrm{~m}$, Yungas montane forest, corticolous, 30 June 2010, $A F$ $17223 \& P R$ (LPB).

\section{"Stereocaulon didymicum I. M. Lamb}

Previously reported from Colombia, Costa Rica, Ecuador and Venezuela (Lamb 1977; LópezFigueiras 1986; Sipman 1992b; Sipman et al. 2008).

Specimens eXamined. BOLIVIA. Dept. La PaZ. Prov. Nor Yungas, Serranía Bellavista, ca $36 \mathrm{~km}$ por camino de Caranavi hacia Sapecho, $15^{\circ} 41^{\prime} \mathrm{S}, 67^{\circ} 30^{\prime} \mathrm{W}$, $1500 \mathrm{~m}$, pared rocosa, saxicolous, 31 Aug. 1997, KB et al. 433 (B, LPB).

\section{${ }^{* * *}$ Szczawinskia tsugae A. Funk}

This species is known from North America, with only one record in the Neotropics (Costa Rica) (Funk 1983; Brodo \& Tønsberg 1994; Lücking 2008).

CHEMISTRY. Not tested with TLC due to insufficient material; the thallus did not react with $\mathrm{C}$ and $\mathrm{K}$ (checked in squash preparations).

Specimen examined. BOLIVIA. Dept. La PaZ. Prov. Nor Yungas, $\mathrm{km} 69$ on the road between Coroico and La Paz, 16 ${ }^{\circ} 13^{\prime} 33^{\prime \prime} \mathrm{S}, 67^{\circ} 49^{\prime} 20^{\prime \prime} \mathrm{W}, 2600 \mathrm{~m}$, Yungas secondary cloud forest, on bark, 23 May 2011, $M K$ $9177 b$ (LPB).

\section{${ }^{*}$ Tetramelas thiopolizus (Nyl.) Giralt \& P. Clerc}

This lichen is known from Chile and Venezuela in South America, and elsewhere from Asia (Siberia) and Europe (Austria, France, Germany, the Iberian Peninsula, Italy) (Giralt \& Clerc 2011).

SPECIMENS EXAMINED. BOLIVIA. DEPT. ORURO. Prov. Sajama, SAJAMA, ladera de la quebrada Kohiri, $18^{\circ} 07^{\prime} 07^{\prime \prime} \mathrm{S}, 68^{\circ} 56^{\prime} 06^{\prime \prime} \mathrm{W}, 4309 \mathrm{~m}$, bosque de Polylepis tarapacana en ladera con piedras grandes, saxicolous, 21 Nov. 2007, RIM et al. 4376 (B, LPB).

\section{${ }^{*}$ Toninia bullata (Meyen \& Flot.) Zahlbr.}

This species seems to be widespread (Lesotho, Australia, New Zealand, Oceania, the U.S.A., Mexico, South America), but rare (Timdal 1992; Elix \& McCarthy 1998; Galloway 2007). Its South American distribution includes Argentina, Chile, Ecuador, Peru and Venezuela (Timdal 1992; Calvelo \& Liberatore 2002; Galloway 2007).

SPECIMENS EXAMINED. BOLIVIA. DEPT. ORURo. Prov. Sajama, SAJAMA, ladera de la quebrada Kohiri, $18^{\circ} 07^{\prime} 07^{\prime \prime} \mathrm{S}, 68^{\circ} 56^{\prime} 06^{\prime \prime} \mathrm{W}, 4309 \mathrm{~m}$, bosque de Polylepis tarapacana en ladera con piedras grandes, saxicolous, 21 Nov. 2007, RIM et al. 4374 (B, LPB).

\section{${ }^{*}$ Toninia cinereovirens (Schaer.) A. Massal.}

Known mainly from Europe and Asia, this lichen has also been reported from scattered localities in Africa, North and South America including Argentina, Peru and Venezuela (Timdal 1992, 2002).

Specimens examined. BOLIVIA. Dept. La Paz. Prov. Camacho, cerca de la comunidad de Ulla Ulla, en el cerro Wilamuku, $15^{\circ} 02^{\prime} 04.1^{\prime \prime} \mathrm{S}, 69^{\circ} 11^{\prime} 52.7^{\prime \prime} \mathrm{W}$, $4739 \mathrm{~m}$, vegetación Altoandina, terricolous, 4 Dec. 2007, $P R 274 a$ (B, LPB).

\section{*Trichothelium caudatum Lücking}

Previously reported from the type locality in Peru (Lücking \& Cáceres 2004). Here it is reported for the first time from Bolivia and Ecuador. 
Specimens eXAmined. BOLIVIA. DePt. LA PAZ. Prov. Larecaja, between Tipuani and Guanay, $15^{\circ} 31^{\prime} 24^{\prime \prime} \mathrm{S}, 67^{\circ} 58^{\prime} 06^{\prime \prime} \mathrm{W}, 1200 \mathrm{~m}$, Preandean Amazon forest, on corticolous bryophytes, 24 Nov. 2011, $M K$ 10419 (LPB, UGDA); DEPT. SANTA CRUZ. Prov. Ca-

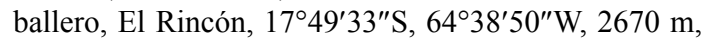
Yungas cloud forest, on bark, 17 Aug. 2012, MK 11545 (LPB). ECUADOR. Prov. Tungurahua, NP Llanganates, ca $5 \mathrm{~km}$ WSW of cerro Hermoso, $01^{\circ} 14^{\prime} 26^{\prime \prime} \mathrm{S}$, 78 19'44"W, 3685-3690 m, on Polylepis, 19 Mar. 2003, ZP 8425 (PRA).

${ }^{*}$ Trichothelium horridulum (Müll. Arg.) R. Sant.

This species is widely distributed in Central and South America (Brazil, Costa Rica, Guyana, Paraguay and Uruguay; Lücking \& Cáceres 2004).

SPECIMENS EXAMINED. BOLIVIA. DEPT. COCHABAMBA. Prov. Chapare, PN Carrasco, near Incachaca, $17^{\circ} 14^{\prime} 11^{\prime \prime} \mathrm{S}, 65^{\circ} 49^{\prime} 02^{\prime \prime} \mathrm{W}, 2317 \mathrm{~m}$, Pinus plantation near Yungas cloud forest, corticolous, 12 June 2006, AF 8349 (LPB).

\section{${ }^{* *}$ Trinathotrema lumbricoides (Sipman) Sipman \& Aptroot}

Previously reported from Japan, Papua New Guinea, Thailand and Vietnam (Aptroot et al. 1997; Wolseley et al. 2002; Lücking et al. 2011b).

Specimens examined. BOLIVIA. Dept. La PaZ. Prov. Iturralde, between Ixiamas and Santa Rosa de Maravillas, $13^{\circ} 49^{\prime} 16^{\prime \prime} \mathrm{S}, 68^{\circ} 07^{\prime} 18^{\prime \prime} \mathrm{W}, 305 \mathrm{~m}$, Subandean Amazon forest, on twig, 28 July 2008, MK 6834 (LPB, UGDA).

\section{Trypethelium aeneum (Eschw.) Zahlbr.}

This species was only recently reported from three localities in Bolivia (Flakus et al. 2012a).

CHEMISTRY. Unidentified $\mathrm{K}+$ purple and UV+ orange-red anthraquinone on pseudostroma.

Specimens eXamined. BOLIVIA. Dept. Beni. Prov. General José Ballivián, Palmar, 14 ${ }^{\circ} 58^{\prime} 56^{\prime \prime} \mathrm{S}$, $67^{\circ} 05^{\prime} 16^{\prime \prime} \mathrm{W}, 250 \mathrm{~m}$, by the road, on bark of tree, 2 Aug. 2008, MK 7113 (LPB, UGDA); north of San Borja, $14^{\circ} 46^{\prime} 07^{\prime \prime} \mathrm{S}, 66^{\circ} 46^{\prime} 51^{\prime \prime} \mathrm{W}, 190 \mathrm{~m}$, lowland Amazon forest surrounded by los Llanos de Moxos savanna, on bark, 3 Sept. 2012, MK 12047 (LPB, UGDA); Prov. Marbán, near road from Camiaco to San Lorenzo, $15^{\circ} 14^{\prime} 49^{\prime \prime} \mathrm{S}, 64^{\circ} 49^{\prime} 11^{\prime \prime} \mathrm{W}, 160 \mathrm{~m}$, lowland Amazon forest surrounded by Los Llanos de Moxos savanna, on bark, 30 Aug. 2012, MK $11956 a$ (LPB, UGDA); DEPT. LA PAZ. Prov. Franz Tamayo, between Apolo and Mapiri, $14^{\circ} 41^{\prime} 50^{\prime \prime} \mathrm{S}, 68^{\circ} 25^{\prime} 07^{\prime \prime} \mathrm{W}, 1510 \mathrm{~m}$, savannah with scattered trees, on bark, 22 Nov. 2011, MK 10156 (LPB, UGDA); Prov. Iturralde, near San Pedro, 1343'59"S, $68^{\circ} 00^{\prime} 38^{\prime \prime} \mathrm{W}, 250 \mathrm{~m}$, savanna, on bark of tree, 28 July 2008, MK 6899 (LPB, UGDA); DEPT. SANTA CRUZ. Prov. Andrés Ibáñez, AMBORÓ, on the road to San Mateo de Toresilla, $17^{\circ} 48^{\prime} 11^{\prime \prime} \mathrm{S}, 64^{\circ} 39^{\prime} 28^{\prime \prime} \mathrm{W}, 2250 \mathrm{~m}$, Yungas cloud forest, on bark, 9 June 2011, MK 9997 (LPB, UGDA).

\section{${ }^{*}$ Trypethelium eluteriae Spreng.}

Pantropical species. Its South American distribution includes Brazil, Ecuador (the Galapagos Islands), French Guiana, Paraguay and Venezuela (Malme 1924; López-Figueiras 1986; Hekking \& Sipman 1988; Marcano et al. 1996; Cáceres 2007; Aptroot et al. 2008; Bungartz et al. 2013).

CHEMISTRY. Thallus and stroma $\mathrm{K}-$ and UV-, but an orange-yellow pigment reacting $\mathrm{K}+$ purple is present in the pseudostroma.

Specimens examined. BOliVia, Dept. Beni. Prov. General José Ballivián Segurola, north of San

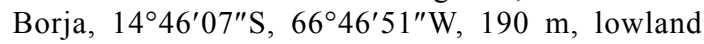
Amazon forest surrounded by los llanos de Moxos savanna, on bark, 3 Sept. 2012, MK 12057 (LPB, UGDA); Prov. Marbán, near road from Camiaco to San Lorenzo, $15^{\circ} 14^{\prime} 49^{\prime \prime} \mathrm{S}, 64^{\circ} 49^{\prime} 11^{\prime \prime} \mathrm{W}, 160 \mathrm{~m}$, lowland Amazon forest surrounded by los Llanos de Moxos savanna, on tree bark, 30 Aug. 2012, MK 11955 (LPB,

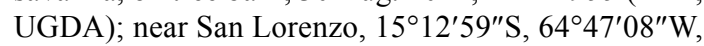
$160 \mathrm{~m}$, lowland Amazon forest surrounded by los Llanos de Moxos savanna, on bark, 28 Aug. 2012, MK 11894 (LPB, UGDA); DePT. SANTA CRUZ. Prov. Cordillera, $350 \mathrm{~km}$ on the road from Tucavaca to Roboré, $18^{\circ} 37^{\prime} 56^{\prime \prime} \mathrm{S}, 59^{\circ} 36^{\prime} 50^{\prime \prime} \mathrm{W}, 370 \mathrm{~m}$, transition ChiquitanoChaqueño forest, on twigs, 4 Dec. 2011, MK 10531 \& 10515 (LPB, UGDA); SSW of Roboré, 18 $36^{\prime} 11^{\prime \prime} \mathrm{S}$, $59^{\circ} 53^{\prime} 06^{\prime \prime} \mathrm{W}, 320 \mathrm{~m}$, transition Chiquitano-Chaqueño forest, 4 Dec. 2011, MK 10607 (LPB, UGDA); near Peto Blanco, $18^{\circ} 48^{\prime} 25^{\prime \prime} \mathrm{S}, 60^{\circ} 14^{\prime} 54^{\prime \prime} \mathrm{W}, 330 \mathrm{~m}$, transition Chiquitano-Chaqueño forest, on bark, 6 Dec. 2011, MK 10678 (LPB, UGDA); KAA-IYA, by Peto Blanco, $18^{\circ} 51^{\prime} 36^{\prime \prime} \mathrm{S}, 60^{\circ} 18^{\prime} 00^{\prime \prime} \mathrm{W}, 310 \mathrm{~m}$, transition Chiquitano-Chaqueño forest, on tree bark, 5 Dec. 2011, $M K 10666$ (LPB, UGDA). 


\section{${ }^{*}$ Trypethelium nitidiusculum (Nyl.) R. C. Harris}

This pantropical species is most common in lowland forest (Aptroot et al. 1997, 2008; Elix \& McCarthy 1998; Umaña-Tenorio et al. 2002; Cáceres 2007). In South America it has been found in Brazil, Ecuador (the Galapagos Islands) and Venezuela (Sipman 1992a; Cáceres 2007; Bungartz et al. 2013).

\section{CHEMISTRY. Thallus $\mathrm{K}$ - and UV-.}

Specimens eXamined. BOLIVIA. Dept. Beni. Prov. Marbán, near road from Camiaco to San Lorenzo, $15^{\circ} 14^{\prime} 49^{\prime \prime} \mathrm{S}, 64^{\circ} 49^{\prime} 11^{\prime \prime} \mathrm{W}, 160 \mathrm{~m}$, lowland Amazon forest surrounded by los Llanos de Moxos savanna, on bark, 30 Aug. 2012, MK 11948 (LPB, UGDA); DEPT. LA PAZ. Prov. Nor Yungas, near Pacallo, $16^{\circ} 12^{\prime} 10^{\prime \prime} \mathrm{S}$, $67^{\circ} 50^{\prime} 39^{\prime \prime} \mathrm{W}, 1360 \mathrm{~m}$, montane forest, on tree bark, 3 Aug. 2008, MK 7123 (LPB, UGDA); Coroico, Sol y Luna hotel, $1870 \mathrm{~m}, 16^{\circ} 11^{\prime} 56^{\prime \prime} \mathrm{S}, 67^{\circ} 43^{\prime} 26^{\prime \prime} \mathrm{W}$, gardens with trees, on bark, 13 Dec. 2011, MK 10739 (LPB, UGDA).

\section{Trypethelium ochroleucum (Eschw.) Nyl.}

Previously reported from two localities in Bolivia (Flakus \& Kukwa 2012b; Flakus et al. 2012a).

\section{CHEMISTRY. Thallus $\mathrm{K}-$, UV+ orange.}

SPECIMENS EXAMINED. BOLIVIA. DEPT. BENI. Prov. Cercado, Isla de los Caimánes, near San Pedro Nuevo, $14^{\circ} 30^{\prime} 11^{\prime \prime} \mathrm{S}, 64^{\circ} 52^{\prime} 21^{\prime \prime} \mathrm{W}, 155 \mathrm{~m}$, lowland Amazon forest surrounded by los Llanos de Moxos savanna, by lake, on bark, 1 Sept. 2012, MK $12005 a$ (LPB, UGDA); Casa del Tigre near Chuchini, $14^{\circ} 43^{\prime} 17^{\prime \prime} \mathrm{S}, 64^{\circ} 56^{\prime} 53^{\prime \prime} \mathrm{W}, 160 \mathrm{~m}$, lowland Amazon forest, on bark, 31 Aug. 2012, $M K$ 11968 (LPB, UGDA); Prov. General José Ballivián Se-

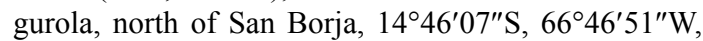
$190 \mathrm{~m}$, lowland Amazon forest surrounded by los Llanos de Moxos savanna, on bark, 3 Sept. 2012 MK 12042 (LPB, UGDA); Prov. Mamoré, near La Esperanza, $13^{\circ} 55^{\prime} 26^{\prime \prime} \mathrm{S}, 64^{\circ} 59^{\prime} 46^{\prime \prime} \mathrm{W}, 140 \mathrm{~m}$, los Llanos de Moxos savanna by lake, on bark, 1 Sept. 2012. MK 12009 \& 12007; Prov. Marbán, near road from Camiaco to

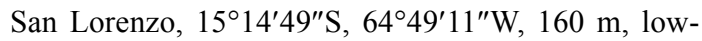
land Amazon forest surrounded by los Llanos de Moxos savanna, on bark, 30 Aug. 2012, MK 11956 (LPB, UGDA); near San Lorenzo, $15^{\circ} 12^{\prime} 59^{\prime \prime} \mathrm{S}, 64^{\circ} 47^{\prime} 08^{\prime \prime} \mathrm{W}$, $160 \mathrm{~m}$, lowland Amazon forest surrounded by los Llanos de Moxos savanna, on bark, 28 Aug. 2012, MK 11893 (LPB, UGDA).
${ }^{*}$ Trypethelium tropicum (Ach.) Müll. Arg.

This is a widely distributed lichen, though not recorded so far from Africa (Aptroot \& Sipman 1991, 2001; Harris 1995; Elix \& McCarthy 1998; Cáceres 2007). In South America it has been reported from Brazil, Ecuador (the Galapagos Islands), French Guiana, Guyana, Paraguay and Venezuela (Müller 1888; Malme 1924; López-Figueiras 1986; Hekking \& Sipman 1988; Marcano et al. 1996; Cáceres 2007; Bungartz et al. 2013).

CHEMISTRY. Thallus and pseudostroma $\mathrm{K}-$ and $\mathrm{UV}-$.

Specimens examined. BOLIVIA. Dept. La PaZ. Prov. Nor Yungas, near Pongo, near the road from Coroico to $\mathrm{La} \mathrm{Paz}, 16^{\circ} 19^{\prime} 28^{\prime \prime} \mathrm{S}, 67^{\circ} 57^{\prime} 21^{\prime \prime} \mathrm{W}, 3822 \mathrm{~m}$, Páramo Yungueño, on bark, 26 Nov. 2011, MK 10495 (LPB, UGDA).

Varicellaria velata (Turner) Schmitt \& Lumbsch

Pertusaria velata (Turner) Nyl.

This is the second record of this species from Bolivia (Flakus \& Kukwa 2012a).

ChEMISTRY. Not tested by TLC; thallus UV-, $\mathrm{C}+$ carmine-red.

Specimen eXamined. BOLIVIA. Dept. LA PaZ. Prov. Franz Tamayo, between Apolo and Mapiri, $15^{\circ} 01^{\prime} 50^{\prime \prime} \mathrm{S}, 68^{\circ} 18^{\prime} 33^{\prime \prime} \mathrm{W}, 1120 \mathrm{~m}$, Preandean Amazon forest, on bark, 23 Nov. 2011, MK $10326 a$ (LPB).

Xanthoparmelia callifolioides Adler, Elix \& J. Johnst.

Previously reported from two localities in Bolivia (Nash et al. 1995; Feuerer \& Sipman 2005).

CHEMISTRY. Usnic, salazinic and consalazinic $( \pm)$ acids.

Specimens eXAmined. BOLIVIA. Dept. LA PAZ. Prov. Murillo, Valle de la Luna, near Mallasa district, $16^{\circ} 34^{\prime} 03^{\prime \prime} \mathrm{S}, 6^{\circ} 05^{\prime} 38^{\prime \prime} \mathrm{W}, 3350 \mathrm{~m}$, open semi-desert high Andean area, on soil, 17 Nov. 2009, AF 14652 \& PR (KRAM, LPB); Prov. Omasuyos, Tajani, cerca Achacachi, $15^{\circ} 36^{\prime} 49^{\prime \prime} \mathrm{S}, 69^{\circ} 04^{\prime} 02^{\prime \prime} \mathrm{W}, 3869$ m, Puna Húmeda, saxicolous, 6 July 2010, AF 17798, 17804 \& PR (LPB, herb. Flakus). 
Xanthoparmelia conspersa (Ehrh. ex Ach.) Hale

This species has been reported in Bolivia only by Nylander (1861). acids.

Chemistry. Usnic, norstictic and stictic

SPECIMENS EXAMINED. BOLIVIA. DePT. ORURO. Prov. Sajama, SAJAMA, Jecha K'ala, $18^{\circ} 09^{\prime} 52^{\prime \prime}$, $68^{\circ} 49^{\prime} 08^{\prime \prime} \mathrm{W}, 4184 \mathrm{~m}$, Puna Sureña, grassland vegetation, on siliceous rock, 20 June 2010, AF 16622, 16708 \& PR (KRAM, LPB, herb. Flakus).

\section{*Xanthoparmelia cordillerana (Gyeln.) Hale}

This lichen is known from Australia and South America (Argentina, Chile, Ecuador, Peru and Uruguay; Nash et al. 1995).

CHEMISTRY. Usnic, salazinic, consalazinic $( \pm)$ and fatty $( \pm)$ acids.

SPECIMENS EXAMINED. BOLIVIA. DEPT. ORURO. Prov. Sajama, SAJAMA, Jecha K'ala, $18^{\circ} 09^{\prime} 52^{\prime \prime} \mathrm{S}$, $68^{\circ} 49^{\prime} 08^{\prime \prime} \mathrm{W}, 4184 \mathrm{~m}$, Puna Sureña, grassland vegetation, on volcanic rock, 20 June 2010, $A F 16632 \& P R$ (KRAM); DEPT. Potosí. Prov. Nor Lípez, Pinturas Rupestres, near Mallku Villamar, $21^{\circ} 46^{\prime} 20^{\prime \prime} \mathrm{S}, 67^{\circ} 29^{\prime} 05^{\prime \prime} \mathrm{W}$, $4038 \mathrm{~m}$, open semi-desert high Andean area, on volcanic rock, 6 Dec. 2009, $A F 14758 \& P R$ (LPB, herb. Flakus).

Xanthoparmelia ferraroiana T. H. Nash, Elix \& J. Johnst.

This South American species is already known from one Bolivian locality (Nash et al. 1995).

CHEMISTRY. Usnic, psoromic (major), 2-Odemethylpsoromic (trace) and fumarprotocetraric (minor) acids.

Specimen eXAmined. BOLIVIA. Dept. Potosí. Prov. Daniel Campos, Puerto Chuvica, near Salar Uyuni, $20^{\circ} 36^{\prime} 06^{\prime \prime} \mathrm{S}, 67^{\circ} 35^{\prime} 50^{\prime \prime} \mathrm{W}, 3670 \mathrm{~m}$, open semi-desert high Andean area, on volcanic rock, 4 Dec. 2009, $A F$ $14692 \& P R$ (LPB).

\section{*Xanthoparmelia hypopsila (Müll. Arg.) Hale}

This widespread lichen was reported in South America from Argentina, Brazil, Chile, Paraguay,
Peru, Uruguay and Venezuela (Hale 1990b; Nash et al. 1995; Feuerer 2013).

CHEMISTRY. Usnic, stictic and norstictic (trace) acids.

Specimens examined. BOLIVIA. DePt. La PaZ.

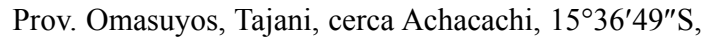
$69^{\circ} 04^{\prime} 02^{\prime \prime} \mathrm{W}, 3869 \mathrm{~m}$, Puna Húmeda, terricolous, 6 July 2010, $A F 17793 \&$ \& $R$ (KRAM, LPB).

\section{${ }^{*}$ Xanthoparmelia lineola (E. C. Berry) Hale}

This lichen is known from Australia, China, South Africa and North and South America (Argentina and Peru) (Hale 1990b; Wei 1991; Nash et al. 1995).

CHEMISTRY. Atranorin, usnic, salazinic, consalazinic, norstictic (trace) and protocetraric (trace).

SpeCimens EXAMined. BOLIVIA. DePt. SANTA Cruz. Prov. Caballero, East Cordillera, Siberia, $17^{\circ} 49^{\prime} 38^{\prime \prime} \mathrm{S}, 64^{\circ} 45^{\prime} 14^{\prime \prime} \mathrm{W}, 3480 \mathrm{~m}$, open area near Yungas montane cloud forest, on siliceous sandstone, 15 Dec. 2004, AF 4837 (LPB, KRAM).

Xanthoparmelia microspora (Müll. Arg.) Hale

This species has been reported from Bolivia by Hale (1990b) and Nash et al. (1995).

CHEMISTRY. Usnic, salazinic and consalazinic $( \pm)$ acids.

SPECIMENS EXAMINED. BOLIVIA. DEPT. COCHABAMBA. Prov. Chapare, cerca de la represa Corani, al lado del camino, on rock, $3250 \mathrm{~m}, 27$ Sept. 1988, S. Arrazola 54 (LPB); DEPT. LA PAZ. Prov. Camacho, Pacoamba, near Wila Kala, $15^{\circ} 24^{\prime} 40^{\prime \prime} \mathrm{S}, 69^{\circ} 04^{\prime} 24^{\prime \prime} \mathrm{W}$, $4283 \mathrm{~m}$, Puna Húmeda vegetation, on saxicolous bryophytes, 6 July 2010, AF 17704.2 \& PR (LPB); Prov. Los Andes, $10 \mathrm{~km}$ del camino Tiahuanacu-La Paz, $3970 \mathrm{~m}$, on siliceous sandstone, 14 June 1981, M. Liberman 392 (LPB); DEPT. ORURO. Prov. Sajama, Parque Nacional Sajama, Jecha K'ala, $18^{\circ} 09^{\prime} 52^{\prime \prime} \mathrm{S}, 68^{\circ} 49^{\prime} 08^{\prime \prime} \mathrm{W}, 4184 \mathrm{~m}$, Puna Sureña, grassland vegetation, on volcanic rock, 20 June 2010, $A F 16710 \& P R$ (KRAM).

\section{*Xanthoparmelia neotaractica Hale}

This is the second locality of this species in South America (Nash et al. 1995); it is also known 
from southeastern U.S.A. (Hale 1984, 1990b; Nash et al. 1995).

CHEMISTRY. Usnic, stictic, constictic and norstictic (minor) acids.

Specimen examined. BOliVia. Dept. CochaBAMBA. Prov. Ayopaya, $20 \mathrm{~km}$ de Cocapata hacia Cotacajes, $16^{\circ} 46^{\prime} \mathrm{S}, 66^{\circ} 44^{\prime} \mathrm{W}, 2000 \mathrm{~m}$, bosque semideciduo intervenido, exposición W, on soil, 15 May 1997, KB et al. 114 (LPB).

\section{${ }^{*}$ Xanthoparmelia plittii (Gyeln.) Hale}

A widely distributed species (Hale 1990b; Nash et al. 1995; Hawksworth et al. 2008), its South American range includes Argentina, Brazil, Colombia, Par aguay, Uruguay and Venezuela (Hale 1990b; Nash et al. 1995).

CHEMISTRY. Usnic acid and the stictic acid complex with norstictic acid.

Specimens examined. BOLIVIA. Dept. La PaZ. Prov. Murillo, TUNI-CONDORIRI, Comunidad Tuni, vegetación Altoandina, $16^{\circ} 13^{\prime} 28^{\prime \prime} \mathrm{S}, 68^{\circ} 16^{\prime} 03^{\prime \prime} \mathrm{W}$, on siliceous schist, 17 March 2009, PR 994 (LPB); DEPT. LA PAZ. Prov. Bautista Saavedra, APOLOBAMBA, near Taypi Cañuma, $15^{\circ} 03^{\prime} 20^{\prime \prime} \mathrm{S}, 6^{\circ} 09^{\prime} 07^{\prime \prime} \mathrm{W}, 4506 \mathrm{~m}$, high Andean open vegetation, saxicolous, 5 July 2010, $A F$ 17492, 17556 \& PR (KRAM, LPB, herb. Flakus); DEPT. ORURo. Prov. Sajama, SAJAMA, Jecha K'ala, $18^{\circ} 09^{\prime} 52^{\prime \prime} \mathrm{S}, 68^{\circ} 49^{\prime} 08^{\prime \prime} \mathrm{W}, 4184 \mathrm{~m}$, Puna Sureña, grassland vegetation, saxicolous, 20 June 2010, $A F$ $16686 \&$ PR (KRAM).

\section{${ }^{*}$ Xanthoparmelia sipmanii T. H. Nash \& Elix}

This endemic South American species has been reported from Brazil, Paraguay and Venezuela (Nash et al. 1995).

Chemistry. Usnic and protocetraric acids.

SPECIMENS EXAMINED. BOLIVIA. DEPT. ORURo. Prov. Sajama, SAJAMA, Huincurata, near Sajama, $18^{\circ} 07^{\prime} 01^{\prime \prime} \mathrm{S}, 68^{\circ} 58^{\prime} 00^{\prime \prime} \mathrm{W}, 4301 \mathrm{~m}$, Puna Sureña, Polylepis forest, saxicolous, 19 June 2010, AF 16594 \& PR (KRAM, LPB); DEPT. Potosí. Prov. Nor Lípez, Pinturas Rupestres, near Mallku Villamar, $21^{\circ} 46^{\prime} 20^{\prime \prime} \mathrm{S}$, $67^{\circ} 29^{\prime} 05^{\prime \prime} \mathrm{W}, 4038 \mathrm{~m}$, open semi-desert high Andean area, on volcanic rock, 6 Dec. 2009, AF $14776 \& P R$ (KRAM, LPB).

\section{*Xanthoparmelia subsorediata Hale}

This lichen is known only from Brazil, Chile, Ecuador and Venezuela (Nash et al. 1995).

CHEMISTRY. Usnic, norstictic, stictic and connorstictic acids.

Specimens eXamined. BOLIVIA. DePt. LA PaZ. Prov. Bautista Saavedra, APOLOBAMBA, near Taypi Cañuma, $15^{\circ} 03^{\prime} 20^{\prime \prime} \mathrm{S}, 6^{\circ} 09^{\prime} 07^{\prime \prime} \mathrm{W}, 4506 \mathrm{~m}$, high Andean open vegetation, saxicolous, 5 July 2010, AF 17502 \& PR (KRAM, LPB).

${ }^{*}$ Xanthoparmelia subulcerosa T. H. Nash \& Elix

A South American species, X. subulcerosa is already known from Argentina, Colombia, Ecuador and Venezuela (Nash et al. 1995; Calvelo \& Liberatore 2002).

CHEMISTRY. Usnic and salazinic acids with a trace of norstictic acid.

Specimens examined. BOLIVIA. Dept. La Paz. Prov. Murillo, La Paz, Cota Cota Calle 27, University Campus, Herbario Nacional de Bolivia, $16^{\circ} 32^{\prime} 16^{\prime \prime} \mathrm{S}$, $68^{\circ} 04^{\prime} 08^{\prime \prime} \mathrm{W}, 3650 \mathrm{~m}$, Botanical Garden, on rock, 28 June 2008, MK 5984 (LPB, UGDA).

*Xanthoparmelia xanthomelaena (Müll. Arg.) Hale

This lichen has been reported from Argentina, Brazil and Venezuela, as well as Australia and South Africa (Hale 1976b; Nash et al. 1995; Triebel et al. 1995).

CHEMISTRY. Usnic, norstictic (trace) and stictic acids.

Specimens examined. BOLIVIA. Dept. La PaZ. Prov. Franz Tamayo, APOLOBAMBA, near Puyo Puyo, $14^{\circ} 56^{\prime} 55^{\prime \prime} \mathrm{S}, 69^{\circ} 07^{\prime} 58^{\prime \prime} \mathrm{W}, 4888 \mathrm{~m}$, high Andean open vegetation, on schist rock, 5 July 2010, AF 17596.1 $\& P R$ (KRAM, LPB).

ACKNOWLEDGEMENTS. We are greatly indebted to the curators of the herbaria B and LPB for the loan of specimens, and to our colleagues for their kind help during the field work. Special thanks go to the Herbario Nacional de Bolivia, Universidad Mayor de San Andrés and Museo Nacional de Historia Natural La Paz for generous cooperation. We are also grateful to the anonymous reviewer, 
and Mr. Robert Anglin and Dr. Thomas Nash (University of Wisconsin, Madison, WI), for commenting on the manuscript and improving the English. This research received support from the National Centre for Research and Development (NCBiR) in Poland under the LIDER Programme for 2010-2013 (No. 92/L-1/09), and also from the University of Gdańsk and the W. Szafer Institute of Botany of the Polish Academy of Sciences through its statutory funds. ZP acknowledges support from the Academy of Science (AV0Z60050516) and the Ministry of Culture (DF12P01OVV025) of the Czech Republic.

\section{REFERENCES}

Aнti T. 2000. Cladoniaceae. Fl. Neotrop. Monogr. 78: $1-362$.

Amtoft A. 2002. Pyxine subcinerea in the eastern United States. Bryologist 105(2): 270-272.

Aptroot A. 1987. Pyxinaceae (Lichens). Fl. Guianas, Ser. E, Fungi \& Lichens 1: 1-59.

Aptroot A. 1998. A world revision of Massarina (Ascomycota). Nova Hedwigia 66(1-2): 89-162.

Aptroot A. 2002. New and interesting lichens and lichenicolous fungi in Brazil. Fung. Diversity 9: 15-45.

APTroot A. 2009. Trypetheliaceae. In: P. M. MCCARTHY (ed.), Flora of Australia. Vol. 57. Lichens 5: 534-552. ABRS and CSIRO Publishing, Canberra and Melbourne.

Aptroot A. 2012. A world key to the species of Anthracothecium and Pyrenula. Lichenologist 44(1): 5-53.

Aptroot A. \& Sipman H. J. M. 1991. New lichens and lichen records from New Guinea. Willdenowia 20: 221-256.

Aptroot A. \& Sipman H. J. M. 1993. Trichotheliaceae. Fl. Guianas, Ser. E, Fungi \& Lichens 2: 3-56.

Aptroot A. \& Sipman H. J. M. 2001. New Hong Kong lichens, ascomycetes and lichenicolous fungi. J. Hattori Bot. Lab. 91: 317-343.

Aptroot A., Diederich P., SÉRUSiaux E. \& SipMAn H. J. M. 1997. Lichens and lichenicolous fungi from New Guinea. Biblioth. Lichenol. 64: 1-220.

Aptroot A., Herk van K., Sparrius L. \& BoOM VAN DEN P. 1999. Checklist van de Nederlandse lichenen en lichenicole fungi. Buxbaumiella 50: 4-64.

Aptroot A., SAipunkaew W., Sipman H. J. M., Sparrius L. B. \& Wolseley P. A. 2007. New lichens from Thailand, mainly microlichens from Chiang Mai. Fung. Diversity 24: 75-134.

Aptroot A., LÜCKING R., SipMAn H. J. M., UMAÑA L. \& CHAVES J. L. 2008. Pyrenocarpous Lichens with bitunicate asci: a first assessment of the lichen biodiversity inventory in Costa Rica. Biblioth. Lichenol. 97: 1-162.

ARVIDSSON L. 1991. Lichenological studies in Ecuador. In: D. J. Galloway (ed.), Tropical Lichens: Their Systematics, Conservation, and Ecology. Systematics Association Special Volume 43: 123-134. Clarendon Press, Oxford.

Awasthi D. D. 1975. A monograph of the lichen genus Dirinaria. Biblioth. Lichenol. 2: 1-108.

Baloch E., Gilenstam G. \& Wedin M. 2009. Phylogeny and classification of Cryptodiscus, with a taxonomic synopsis of the Swedish species. Fung. Diversity 38: 51-68.

Benatti M. N. \& Marcelli M. P. 2009. Espécies de Parmotrema (Parmeliaceae, Ascomycetes liquenizados) com medula pigmentada do litoral centro-sul do Estado de São Paulo. Hoehnea 36: 597-612.

Benatti M. N. \& Marcelli M. P. 2011. Espécies de Parmotrema (Parmeliaceae, Ascomycota) do litoral Centro-Sul do Estado de São Paulo Iv. Grupo químico protocetrárico. Revista Brasil. Bot. 34: 103-123.

Brako L. 1991. Phyllopsora (Bacidiaceae). Fl. Neotrop. Monogr. 55: 1-66.

BREuss O. 1993. Catapyrenium (Verrucariaceae) species from South America. Pl. Syst. Evol. 185: 17-33.

BREUSS O. 2001. Flechten aus Costa Rica II. Linzer Biol. Beitr. 33(2): 1025-1034.

BReuss O. 2008. Flechten aus Panama. Linzer Biol. Beitr. 40(1): 321-327.

BREUSS O. \& JOHN V. 2004. New and interesting records of lichens from Turkey. Österr. Z. Pilzk. 13: 281-294.

Breuss O. \& NeuwirTh G. 2007. Flechtenfunde im Bosque Esquinas, Costa Rica. Linzer Biol. Beitr. 39(1): 557-569.

Brodo I. M. \& Tønsberg T. 1994. A new species of Micarea with stalked pycnidia from the west coast of North America. Acta Bot. Fenn. 150: 1-4.

Bungartz F., Ziemmeck F., YÁnez AyabacA A., Nugra F. \& APTROOT A. 2013. CDF Checklist of Galapagos Lichenized Fungi. FCD Lista de especies de Hongos liquenizados Galápagos. (Last updated: 6 June 2013). http://checklists. datazone.darwinfoundation.org/true-fungi/lichens.

CÁCERES M. E. S. 2007. Corticolous crustose and microfoliose lichens of northeastern Brazil. Libri Botanici 22: 1-168.

Calvelo S. \& Liberatore S. 2002. Catálogo de los líquenes de la Argentina. Kurtziana 29(2): 7-170.

Canseco A., Anze R. \& Franken M. 2006. Comunidades de líquenes: indicadores de la calidad del aire en la ciudad de La Paz, Bolivia. Acta Nova 3(2): 286-307.

Chaves J. L., LÜCKING R. SiPMAN H. J. M, UMAÑA L. \& NAVARRO E. 2004. A first assessment of the Ticolichen biodiversity inventory in Costa Rica: the genus Dictyonema (Polyporales: Atheliaceae). Bryologist 107(2): 242-249. 
Culberson C. F. \& Kristinsson H. 1970. A standardized method for the identification of lichen products. J. Chromatogr. 46: 85-93.

COPPINS B. J. 2002. Checklist of lichens of Great Britain and Ireland. British Lichen Society, London.

Culberson W. L. \& Hale M. E. 1965. Pyxine caesiopruinosa in the United States. Bryologist 68(1): 113-116.

CZyŻewsKa K. \& KuKWA M. 2005. Notes on two species of Lepraria from Belarus. Graphis Scripta 17(1): 20-21.

Dodge C. W. 1953. Some lichens from tropical Africa. Ann. Missouri Bot. Gard. 40(4): 271- 401.

Elix J. A. 2009a. Stereocaulaceae. In: P. M. MCCARTHY (ed.), Flora of Australia. Vol. 57. Lichens 5: 60-73. ABRS and CSIRO Publishing, Canberra and Melbourne.

EliX J. A. 2009b. Phyllopsoraceae. In: P. M. MCCARTHY (ed.), Flora of Australia. Vol. 57. Lichens 5: 41-59. ABRS and CSIRO Publishing, Canberra and Melbourne.

EliX J. A. 2009c. Physciaceae. In: P. M. MCCARTHY (ed.), Flora of Australia. Vol. 57. Lichens 5: 494-533. ABRS and CSIRO Publishing, Canberra and Melbourne.

EliX J. A. \& MCCARThy P. M. 1998. Catalogue of the lichens of the smaller Pacific islands. Biblioth. Lichenol. 70: $1-361$.

EliX J. A. \& NASH III T. H. 1992. A synopsis of the lichen genus Psiloparmelia (Ascomycotina, Parmeliaceae). Bryologist 95(4): 377-391.

EsSLinger T. L. 1977. Studies in the lichen family Physiaceae. I. A new North American species. Mycotaxon 5: 299-306.

ESSLINGER T. L. 2004. Phaeophyscia. In: T. H. NASH III, B. D. RYAN, P. DiEDERICH, C. GRIES \& F. Bungartz (eds), Lichen flora of the Greater Sonoran Desert Region 2: 403-414. Lichens Unlimited, Arizona State University, Tempe.

ESSLINGER T. L. 2012. A cumulative checklist for the lichenforming, lichenicolous and allied fungi of the continental United States and Canada. North Dakota State University, Fargo, North Dakota. [Version (\#18) 13 December 2012]. http://www.ndsu.edu/pubweb/ esslinge/chcklst/chcklst7. htm.

ETAYO J. 2002. Aportación al conocimiento de los hongos liquenícolas de Colombia. Biblioth. Lichenol. 84: 1-154.

Ferraro L. I. 2002. Contribution to the knowledge of foliicolous lichens of Bolivia. Mitt. Inst. Allg. Bot. Hamburg 30-32: 47-48.

Ferraro L. I. \& Michlig A. 2013. New species and additional records of Coenogonium (Ostropales: Coenogoniaceae) from southern South America. Lichenologist 45(4): 497-504.

FEUERER T. (ed.) 2010. Checklists of lichens and lichenicolous fungi. Version 1 June 2010. http://www.checklists.de.
FEUERER T. (ed.) 2013. Checklists of lichens and lichenicolous fungi. Version 1 August 2013. http://www.checklists.de.

Feuerer T. \& Sipman H. J. M. 2005. Additions to the lichenized and lichenicolous fungi of Bolivia. Herzogia 18: $139-144$

Feuerer T., Ahti T. \& Vitikainen O. 1998. Lichenological investigations in Bolivia. In: M. P. MARCELLI \& M. R. D. SEAWARD (eds), Lichenology in Latin America: history, current knowledge and applications, pp. 71-86. CETESB, São Paulo.

FlaKUS A. 2008. Contributions to the knowledge of the lichen biota of Bolivia. 2. Polish Bot. J. 53(2): 145-153.

FlakUs A. \& KUKWA M. 2007. New species and records of Lepraria (Stereocaulaceae, lichenized Ascomycota) from South America. Lichenologist 39(5): 463-474.

FlaKUS A. \& KUKWA M. 2012a. New records of lichenicolous fungi from Bolivia. Opuscula Philolichenum 11: 36-48.

FlaKUS A. \& KUKWA M. 2012b. New species of lichenicolous fungi from Bolivia. Lichenologist 44(4): 469-477.

FLAKUS A. \& LÜCKING R 2008. New species and additional records of foliicolous lichenized fungi from Bolivia. $\mathrm{Li}$ chenologist 40(5): 423-436.

FLAKUS A. \& WILK K. 2006. Contribution to the knowledge of the lichen biota of Bolivia. J. Hattori Bot. Lab. 99: 307-318.

Flakus A., Rodriguez SaAvedra P., Kukwa M. 2012 b. A new species and new combinations and records of $\mathrm{Hy}$ potrachyna and Remototrachyna from Bolivia. Mycotaxon 119: $157-166$.

Flakus A., Elix J. A., Rodriguez P. \& KuKwa M. 2011 b. New species and records of Lepraria (Stereocaulaceae, lichenized Ascomycota) from South America. Lichenologist 43(1): 57-66.

Flakus A., Oset M., JablońSKa A., Rodriguez SaAVEdRa P. \& KUKWA M. 2011a. Contribution to the knowledge of the lichen biota of Bolivia. 3. Polish Bot. J. 56(2): 159-183.

Flakus A., ETAYO J., SchiEFElBEIN U., AHTI T., JABŁoŃSKa A., Oset M., Bach K., Rodriguez Flakus P. \& KuKwa M. 2012a. Contribution to the knowledge of the lichen biota of Bolivia. 4. Polish Bot. J. 53(2): 427-461.

FLEIG M. \& GRÜNINGER W. 2000. Levantamento preliminar dos liquens do Centro de Pesquisas e Conservação da Natureza Pró-Mata, São Francisco de Paula, Rio Grande do Sul, Brasil. Napaea 12: 5-20.

FUNK A. 1983. Szczawinskia, a new genus of the lichen-forming Coelomycetes. Syesis 16: 85-88.

GallowaY D. 2007. Flora of New Zealand lichens. Revised second edition including lichen-forming and lichenicolous fungi. 1 \& 2. Manaaki Whenua Press, Lincoln, New Zealand.

Galloway D. J. \& Quilhot W. 1998. Checklist of Chilean 
lichen-forming and lichenicolous fungi. Gayana Botanica 55(2): 111-185.

GIERL C. \& KALB K. 1993. Die Flechtengattung Dibaeis. Eine Übersicht über die rosafrüchtigen Arten von Baeomyces sens. lat. nebst Anmerkungen zu Phyllobaeis gen. nov. Herzogia 9: 593-645.

Giralt M. \& CleRC P. 2011. Tetramelas thiopolizus comb. nov. with a key to all known species of Tetramelas. Lichenologist 43(5): 417-425.

Goward T., McCunE B. \& Meidinger D. 1994. The lichens of British Columbia. Illustrated keys. Part 1 - Foliose and squamulose species. Special Report Series (British Columbia. Ministry of Forests) 8: 1-181.

HAFELLNER J. 1983 (1981). Monographie der Flechtengattung Letrouitia (Lecanorales, Teloschistineae). Nova Hedwigia 35(4): 645-729.

HAFELLNER J. 1995. A new checklist of lichens and lichenicolous fungi of insular Laurimacaronesia including a lichenological bibliography for the area. Fritschiana 5: $1-132$.

HAFELLNER J. \& TÜRK R. 2001. Die lichenisierten Pilze Österreichs - eine Checkliste der bisher nachgewiesenen Arten mit Verbreitungsangaben. Stapfia 76: 3-167.

Hale M. E. 1976a. A monograph of the lichen genus Bulbothrix Hale (Parmeliaceae). Smithsonian Contrib. Bot. 32: 1-29.

Hale M. E. 1976b. A monograph of the lichen genus Pseudoparmelia Lynge (Parmeliaceae). Smithsonian Contrib. Bot. 31: 1-62.

HALE M. E. 1984. New species of Xanthoparmelia (Vain.) Hale (Ascomycotina: Parmeliaceae). Mycotaxon 20: 73-79.

Hale M. E. 1990a. New species of Parmotrema (Ascomycotina: Parmeliaceae) from tropical America. Biblioth. Lichenol. 38: 109-119.

HALE M. E. 1990b. A synopsis of the lichen genus Xanthoparmelia (Vainio) Hale (Ascomycotina, Parmeliaceae). Smithsonian Contrib. Bot. 74: 1-250.

HARRIS R. C. 1986 (1984). The family Trypetheliaceae (Loculoascomycetes: lichenized Melanommatales) in Amazonian Brazil. Suppl. Acta Amazonica 14(1/2): 55-80.

HARRIS R. C. 1995. More Florida lichens. Including the $10 \varnothing$ tour of the Pyrenolichens. Published by the Author, Bronx, N.Y.

Hawksworth D. L., Blanco O., Divakar P. K., Ahti T. \& CRESPO A. 2008. A first checklist of parmelioid and similar lichens in Europe and some adjacent territories, adopting revised generic circumscriptions and with indications of species distributions. Lichenologist 40: 1-21.

HeKking W. H. A. \& Sipman H. J. M. 1988. The lichens reported from the Guianas before 1987. Willdenowia 17: 193-228.
Herzog T. 1922. Beitrag zur Flechtenflora von Bolivia. Hedwigia 63: 263-268.

Herzog T. 1923. Die Pflanzenwelt der bolivianischen Anden und ihres östlichen Vorlandes. In: A. ENGLER \& O. DRUDE (eds), Die Vegetation der Erde 15: 1-258. Engelmann, Leipzig.

Josse C., Navarro G., Comer P., Evans R., Faber-LanGendoen D., Fellows M., Kittel G., Menard S., Pyne M., Reid M., Schulz K., SnOw K. \& Teague J. 2003. Ecological Systems of Latin America and the Caribbean: A Working Classification of Terrestrial Systems. NatureServe, Arlington.

KalB K. 1987. Brasilianische Flechten. 1. Die Gattung Pyxine. Biblioth. Lichenol. 24: 1-89.

KALB K. 1991. Lichenes Neotropici. Fascikel XI (No. 451475). Neumarkt.

KALB K. 2004. New or otherwise interesting lichens II. Biblioth. Lichenol. 88: 301-329.

KalB K. \& EliX J. A. 1995. The lichen genus Physcidia. Biblioth. Lichenol. 57: 265-296.

KALB K. \& VĚZDA A. 1988. Neue oder bemerkenswerte Arten der Flechtenfamilie Gomphillaceae in der Neotropis. Biblioth. Lichenol. 29: 1-80.

Kalb K., Archer A. W., Sutjaritturakan J. \& BoonPraGOB K. 2009. New or otherwise interesting lichens V. Biblioth. Lichenol. 99: 225-246.

KalB K., BuAruang K., MongKolsuk P. \& BoOnPRagob K. 2012. New or otherwise interesting lichens. VI, including a lichenicolous fungus. Phytotaxa 42: 35-47.

Kashiwadani H. \& KalB K. 1993. The genus Ramalina in Brazil. Lichenologist 25(1): 1-31.

KNUDSEN K. 2007. Acarospora. In: T. H. NASH III, C. GRIES \& F. BUNGARTZ (eds), Lichen flora of the Greater Sonoran Desert Region. 3: 1-38. Lichens Unlimited, Arizona State University, Tempe.

KNUDSEN K. \& EliX J. A. 2007. Lepraria. In: T. H. NASH III, C. GRIES \& F. BUNGARTZ (eds), Lichen flora of the Greater Sonoran Desert Region. 3: 384-388. Lichens Unlimited, Arizona State University, Tempe.

KNUDSEN K., ELIX J. A \& ReEB V. 2008. A preliminary study of the genera Acarospora and Pleopsidium in South America. Opuscula Philolichenum 5: 1-22.

KnUdSEn K., Flakus A. \& KuKwa M. 2012. A contribution to the study of Acarosporaceae in South America. Lichenologist 44: 253-262.

KROG H. \& SwINSCOW T. D. V. 1974. Ramalina species with a hollow thallus (Fistularia) in East Africa. Norweg. $J$. Bot. 21: 111-124.

KRZEWICKA B. \& FLAKUS A. 2010. New records of the genus Umbilicaria (Umbilicariaceae, lichenized Ascomycota) from Bolivia. Cryptogamie, Mycologie 31(4): 441-451. 
Kukwa M. BaCh K., Sipman H. J. M. \& Flakus A. 2012. Thirty-six species of the lichen genus Parmotrema (Lecanorales, Ascomycota) new to Bolivia. Polish Bot. J. 57(1): 243-257.

Kukwa M., Schiefelbein U. \& Flakus A. 2013. A contribution to the lichen family Graphidaceae (Ostropales, Ascomycota) of Bolivia. Herzogia 26(2) (in press).

LAMB I. M. 1977. A conspectus of the lichen genus Stereocaulon (Schreb.) Hoffm. J. Hattori Bot. Lab. 43: 191-355.

Letrouit-Galinou M. A. 1957. Revision monographique du genre Laurera (lichens, Trypetheliacees). Rev. Bryol. Lichénol. 26(3-4): 207-264.

LÓPEZ-FIGUEIRAS M. 1986. Censo de macrolíquenes venezolanos de los estados Falcon, Lara, Merida, Tachira y Trujillo. Facultad de Farmacia, Universidad de Los Andes, Merida.

LÜCKING R. 2008. Foliicolous lichenized fungi. Fl. Neotrop. Monogr. 103: 1-867.

LÜCKING R. \& CÁCERES M. 2004. Corticolous species of Trichothelium (Ascomycota: Porinaceae). Mycol. Res. 108(5): 571-575.

LÜCKING R., SÉRUSIAUX E. \& VĚZDA A. 2005. Phylogeny and systematics of the lichen family Gomphillaceae (Ostropales) inferred from cladistic analysis of phenotype data. Lichenologist 37(2): 123-170.

LÜCKing R., Rivas Plata E., Chaves J. L., Umaña L. \& SiPMAN H. J. M. 2009. How many tropical lichens are there really? Biblioth. Lichenol. 100: 399-418.

LÜCKIng R., Rivas Plata E., Mangold A., SiPman H. J. M., Aptroot A., González R. M., Kalb K., Chaves J. L., Ventura N. \& ESQUiVel R. E. 2011b. Natural history of Nash's Pore Lichens, Trinathotrema (Ascomycota: Ostropales: Stictidaceae). Biblioth. Lichenol. 106: $187-210$.

LÜCKING R., SEAVEY F., COMMON R., BEECHING S. Q., BREUSS O., Buck W. R., Crane L., Hodges M., Hodkinson B. P., LAy E., Lendemer J. C., MCMullin R. T., MerCadoDíaz J. A., Nelsen M. P., Rivas Plata E., SAFraneK W., SANDERS W. B., Schaefer H. P. \& SEAVEY J. 2011a. The lichens of Fakahatchee Strand Preserve State Park, florida: Proceedings from the 18th Tuckerman Workshop. Bull. Florida Mus. Nat. Hist. 49: 127-186.

LuMBSCH H. T. 1989. Die holarktischen Vertreter der Flechtengattung Diploschistes (Thelotremataceae). J. Hattori Bot. Lab. 66: 133-196.

Magnusson A. H. 1929. A monograph of the genus Acarospora. Kungl. Svenska Vetenskapsakad. Avh. Naturskyddsärenden 7: 1-400.

Malme G. O. A. 1924. Die Flechten der ersten Regnellschen Expedition. Astrotheliaceae, Paratheliaceae und Trypetheliaceae. Ark. Bot. 19(1): 1-34.

MARCANO V. \& MoRALES MÉndeZ A. 1993. Ramalina reducta
Krog et Swinscow, especie de liquen de los Andes Venezolano, nueva para America. Ernstia 3: 19-26.

MarCAnO V. \& Morales Méndez A. 1994. El grupo de $R a-$ malina cochlearis Zahlbr. con especial referencia al trópico americano. Trop. Bryol. 9: 187-199.

Marcano V., Morales MÉndez A., SiPMAn H. J. M. \& CALDERON L. 1996. A first checklist of the lichen-forming fungi of the Venezuelan Andes. Trop. Bryol. 12: 193-235.

MCCARTHY P. M. 1993. New records of pyrenocarpous lichens from Australia. Muelleria 8(1): 31-36.

MCCARThy P. M. 2003. Catalogue of the lichen family Porinaceae. Biblioth. Lichenol. 87: 1-164.

MCCARThY P. M. 2013. Checklist of the Lichens of Australia and its Island Territories. Version 17 September 2013. Australian Biological Resources Study, Canberra. http:// www.anbg.gov.au/abrs/lichenlist/introduction.html.

Moberg R. 1990. The lichen genus Physcia in Central and South America. Nord. J. Bot. 10: 319-342.

Moberg R. 1993. The lichen genus Phaeophyscia in South America with special reference to Andean species. Opera Bot. 121: 281-284.

Moberg R. 2011. The lichen genus Heterodermia (Physciaceae) in South America - a contribution including five new species. Nord. J. Bot. 29: 129-147.

MÜLleR J. 1883. Lichenologische Beiträge 18. Flora 66: 243-249.

MÜLleR J. 1885. Pyrenocarpeae Cubenses a cl. C. Wright lectae. Engler Bot. Jahrb. 6: 375-421.

MÜLLER J. 1888. Lichenes Paraguayenses a cl. Balansa lecti et a Prof. Dr Müller elaborati. Rev. Mycol. (Toulouse) 10(40): 177-193.

MÜLLER J. 1895. Lichenes usambarenses. In: A. ENGLER (ed.), Beiträge zur Flora von Afrika. IX. Bot. Jahrb. Syst. 20: 289-298.

NASH III T. H., GRIES C. \& Elix J. A. 1995. A revision of the lichen genus Xanthoparmelia in South America. Biblioth. Lichenol. 56: 1-158.

NeUwiRTh G. 2007. Foliicolous lichens from Venezuela with new and remarkable records. Herzogia 20: 319-326.

NÖSKE N. M. \& Sipman H. J. M. 2004. Cryptogams of the Reserva Biológica San Francisco (Province Zamora-Chinchipe, Southern Ecuador) II. Lichens. Cryptog., Mycol. 25(1): 91-100.

NYLANDER W. 1859. Lichenes in regionibus exoticis quibusdam vigentes exponit synopticis enumerationibus. Ann. Sci. Nat. Bot., Sér. 4 11: 205-264.

NYLANDER W. 1861. Additamentum ad lichenographiam Andium Boliviensium. Ann. Sci. Nat. Bot., Sér. 4 15: 365-382.

NYLANDER W. 1863. Lichenes. In: J. TRIANA \& J. E. PLANCHON 
(eds), Prodromus Florae Novo-Granatensis ou Énumération des plantes de la Nouvelle-Grénade avec description des espèces nouvelles. Ann. Sci. Nat. Bot., Sér. 4 19: 286-382.

Obermayer W., Kalb K., Sipman H. J. M. \& NASH III T. H. 2009. New reports of Culbersonia nubila (Moberg) Essl. from the Tibetan Region, Bolivia, Argentina, Lesotho and South Africa. Lichenologist 41(6): 683-687.

Orange A., James P. W. \& White F. J. 2001. Microchemical methods for the identification of lichens. British Lichen Society, London.

OSORIO H. S. 1972. Contribution to the lichen flora of Uruguay. VII. A preliminary catalogue. Comun. Bot. Mus. Hist. Nat. Montevideo 4(56): 1-46.

OSORIO H. S. 1992. Contribucion a la flora liquénica del Uruguay. XXV. Lichenes publicados entre 1972 a 1991. Anales Mus. Nac. Hist. Nat. Montevideo Ser. 2 8: 43-70.

OSORIO H. S. 2000. Contribution to the lichen flora of Uruguay. XXXII. Lichens from Madeiros ranch, Lavalleja department. Comun. Bot. Mus. Hist. Nat. Montevideo 114: $1-8$.

PARMASTO E. 1978. The genus Dictyonema ("Thelephorolichenes"). Nova Hedwigia 29: 99-144.

Poelt J. \& OBermayer W. 1991. Beiträge zur Kenntnis der Flechtenflora des Himalaya II. Die Gattung Bryonora (Lichenes, Lecanoraceae) zugleich eine Revision aller Arten. Nova Hedwigia 53(1-2): 1-26.

Prieto M., Aragón G. \& Martínez I. 2010. The genus $C a-$ tapyrenium s. lat. (Verrucariaceae) in the Iberian Peninsula and the Balearic Islands. Lichenologist 42(6): 637-684.

Rambold G., Mayrhofer H. \& Matzer M. 1994. On the ascus types in the Physciaceae (Lecanorales). Pl. Syst. Evol. 192: 31-40.

REDINGER K. 1933. Neue und wenig bekannte Flechten aus Brasilien. Hedwigia 73: 54-67.

Rivas Plata E., LÜCKing R., Aptroot A., Sipman H. J. M., Chaves J. L., Umaña L. \& Lizano D. 2006. A first assessment of the Ticolichen biodiversity inventory in Costa Rica: the genus Coenogonium (Ostropales: Coenogoniaceae), with a world-wide key and checklist and a phenotype-based cladistic analysis. Fung. Diversity 23: 255-321.

Rodriguez Flakus P., Flakus A., Kukwa M., LÜCKInG R., IsEla MENeses R., Rivas Plata E., StANTON D., TRUONG C. \& VARGAS R. 2013. Preliminary catalogue of lichens and lichenicolous fungi from Bolivia. W. Szafer Institute of Botany PAS, Krakow. [Version 1.3 (1 August 2013)]. http://botan.botany.pl/lichens-bolivia.

SAag L., SAaG A. \& RAndlane T. 2009. World survey of the genus Lepraria (Stereocaulaceae, lichenized Ascomycota). Lichenologist 41(1): 25-60.

SAntesson R., Moberg R., Nordin A., TønsberG T. \& VI-
TIKAINEN O. 2004. Lichen-forming and lichenicolous fungi of Fennoscandia. Museum of Evolution, Uppsala University, Uppsala.

SIPMAN H. J. M. 1983. A monograph of the lichen family Megalosporaceae. Biblioth. Lichenol. 18: 1-241 + 24 plates.

SIPMAN H. J. M. 1986. Additional notes on the lichen family Megalosporaceae. Willdenowia 15: 557-564.

SiPMAN H. J. M. 1992a. Results of a lichenological and bryological exploration of Cerro Guaiquinima (Guayana Highland, Venezuela). Trop. Bryol. 6: 1-31.

SIPMAN H. J. M. 1992b. The origin of the lichen flora of the Colombian Páramos. In: H. BALslev \& J. L. LuTEYN (eds), Páramo, Andean ecosystem under human influence, pp. 95-109. Academic Press, London.

Sipman H. J. M. 1993. Lichens from Mount Kinabalu. Trop. Bryol. 8: 281-314.

SiPMAn H. J. M., HeKKING W. \& AgUIRRE-C. J. 2008. Checklist of Lichenized and Lichenicolous Fungi from Colombia. Biblioteca José Jerónimo Triana 20. Instituto de Ciencias Naturales, Facultad de Ciencias, Universidad Nacional de Colombia, Bogotá.

SpIElmanN A. A. 2006. Checklist of lichens and lichenicolous fungi of Rio Grande do Sul (Brazil). Caderno de Pesquisa, Sér. Biol. 18(2): 7-125.

Śliwa L., Wilk K., Rodriguez Flakus P. \& Flakus A. 2012. New records of Lecanora for Bolivia. Mycotaxon 121: 385-392.

THIERS B. 2012. Index Herbariorum: A global directory of public herbaria and associated staff. New York Botanical Garden's Virtual Herbarium. http://sweetgum.nybg.org/ih.

TiBELL L. 1991. Revision of some taxa of Caliciales described by W. Nylander. Ann. Bot. Fenn. 28: 117-121.

TiBelL L. 1996. Caliciales. Fl. Neotrop. Monogr. 69: 1-78.

Tibell L. 1998. Crustose mazaediate lichens and the Mycocaliciaceae in temperate South America. Biblioth. Lichenol. 71: $1-107$.

TIMDAL E. 1992. A monograph of the genus Toninia (Lecideaceae, Ascomycetes). Opera Bot. 110: 1-137.

Timdal E. 2002. Toninia. In: T. H. NASH III, B. D. RYAN, C. GRIES \& F. BUNGARTZ (eds), Lichen flora of the Greater Sonoran Desert Region. 1: 488-501. Lichens Unlimited, Arizona State University, Tempe.

TIMDAL E. 2008a. Studies on Eschatogonia (Ramalinaceae) in Peru. Lichenologist 40(1): 31-38.

Timdal E. 2008b. Studies on Phyllopsora (Ramalinaceae) in Peru. Lichenologist 40(4): 337-362.

TøNSBERG T. 1992. The sorediate and isidiate, corticolous, crustose lichens in Norway. Sommerfeltia 14: 1-331.

TønsBerg T. 1998 (1997). Additions to the lichen flora of North America VI. Bryologist 100(4): 522-524. 
Triebel D., Rambold G. \& EliX J.A. 1995. A conspectus of the genus Phacopsis (Lecanorales). Bryologist 98(1): 71-83.

TUCKER S. C. \& HARRIS R. C. 1980: New or noteworthy pyrenocarpous lichens from Louisiana and Florida. Bryologist 83(1): 1-20.

Umaña-Tenorio L. U., SiPman H. J. M. \& LÜCKIng R. 2002. Preliminary checklist of lichens from Costa Rica. Version 1.2 (June 2002). http://archive.fieldmuseum.org/ticolichen/ checklist.html.

Urbanavichus G. 2010. A checklist of the lichen flora of Russia. Nauka, Sankt Petersburg.

VĚZDA A. 1983. Zwei neue Arten der Flechtengattung Gyalideopsis. Mitt. Bot. Staatssamml. München 19: 151-161.

WEBER W.A. 1968. A taxonomic revision of Acarospora, Subgenus Xanthothallia. Lichenologist 4(1): 16-31.
WEDIN M. 1995. The lichen family Sphaerophoraceae (Caliciales, Ascomycotina) in temperate areas of the Southern Hemisphere. Symb. Bot. Upsal. 31(1): 1-102.

WEI J.-C. 1991. An Enumeration of Lichens in China. International Academic Publishers, Beijing.

Westberg M. \& ARUP U. 2011. Candelaria pacifica sp. nova (Ascomycota, Candelariales) and the identity of Candelaria vulgaris. Biblioth. Lichenol. 106: 353-364.

Wolseley P. A., AguirRe-Hudson B. \& MCCARThy P. 2002. Catalogue of the lichens of Thailand. Bull. Nat. Hist. Mus. London 32(1): 13-59.

YÁnez A., Dal-Forno M., Bungartz F., LÜCKIng R. \& LAWREY J. D. 2012. A first assessment of Galapagos basidiolichens. Fung. Diversity 52: 225-244.

ZAHLBRUCKNER A. 1922. Catalogus Lichenum Universalis 1. Gebrüder Borntraeger, Leipzig.

Received 15 October 2013 